\title{
IC-Based Controls for Energy-Efficient Lighting
}

\section{Final Report}

\author{
Contract No. DE-FC26-02NT41252
}

Period Covered: November 15, 2001 - February 15, 2005

Submitted to

NETL ADD DOCUMENT CONTROL BLDG. 921

U.S. DEPARTMENT OF ENERGY

NATIONAL ENERGY TECHNOLOGY LABORATORY

P.O. BOX 10940

PITTSBURGH, PA 15236-0940

Submitted by:

GE Global Research Center

One Research Circle

Niskayuna, NY 12309

Contact: Dr. Richard Zhang

Phone: 518-387-5313

Fax: 518-387-7592

Email: zhang@crd.ge.com

All Authors Listed in Acknowledgements

Submitted in: $\quad$ March 2005 


\section{DISCLAIMER}

"This report was prepared as an account of work sponsored by an agency of the United States Government. Neither the United States Government nor any agency thereof, nor any of their employees, makes any warranty, express or implied, or assumes any legal liability or responsibility for the accuracy, completeness, or usefulness of any information, apparatus, product, or process disclosed, or represents that its use would not infringe privately owned rights. Reference herein to any specific commercial product, process, or service by trade name, trademark, manufacturer, or otherwise does not necessarily constitute or imply its endorsement, recommendation, or favoring by the United States Government or any agency thereof. The views and opinions of authors expressed herein do not necessarily state or reflect those of the United States Government or any agency thereof." 


\section{ABSTRACT}

A new approach for driving high frequency energy saving ballasts is developed and documented in this report. The developed approach utilizes an IC-based platform that provides the benefits of reduced system cost, reduced ballast size, and universal application to a wide range of lamp technologies, such as linear fluorescent lamps (LFL), compact fluorescent lamps (CFL) and high intensity discharge lamps (HID). The control IC chip set developed for the platform includes dual low voltage (LV) IC gate drive that provides gate drive for high and low side power switches in typical ballast circuits, and ballast controller IC that provides control functionalities optimal for different lamps and digital interface for future extension to more sophisticated control and communication. 


\section{TABLE OF CONTENTS}

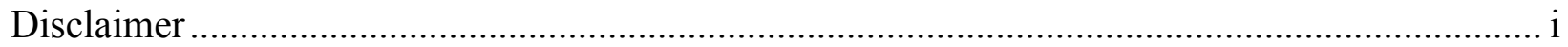

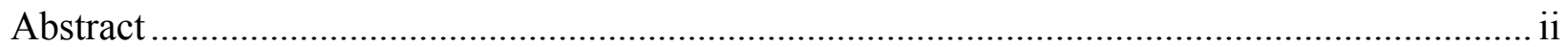

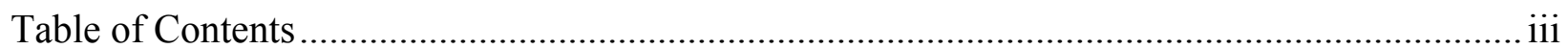

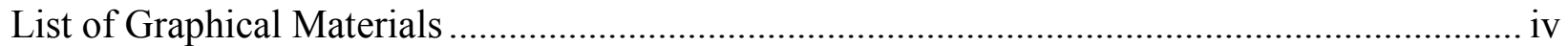

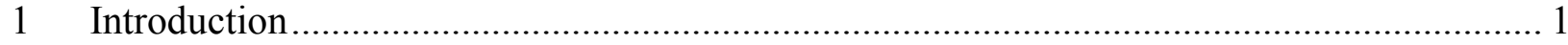

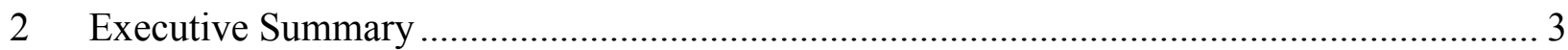

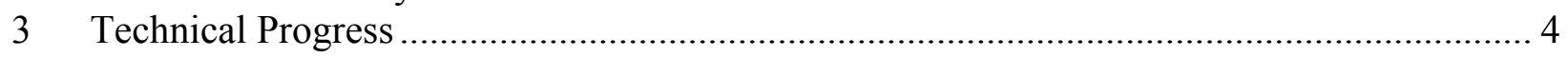

3.1 Dual Low Voltage IC (LVIC) Based Gate Drive ………............................................ 4

3.1.1 Dual LVIC Gate Drive Concept ……….......................................................... 4

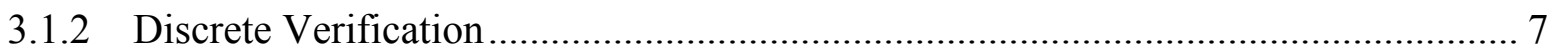

3.1.3 Gate Driver Custom IC Design ......................................................................... 11

3.1.4 Gate Drive Custom IC Simulation Results ……………...................................... 16

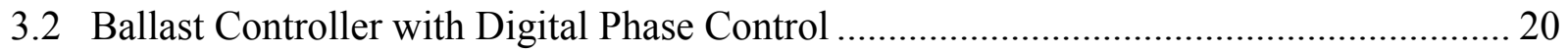

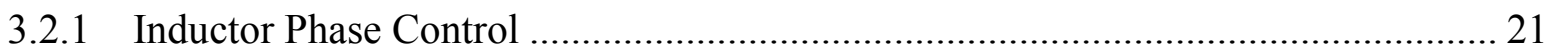

3.2.2 Direct Digital Phase Control Scheme ……....................................................... 22

3.2.3 FPGA-Based Verification of Digital Phase Control............................................... 25

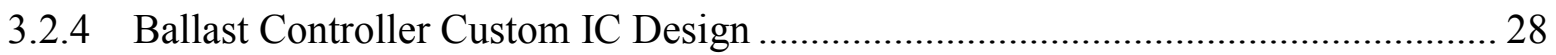

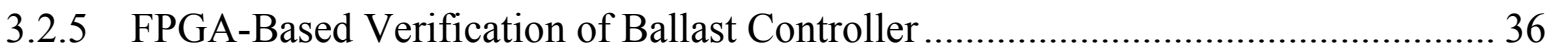

3.2.6 Ballast Controller Custom IC Simulation Results ................................................. 38

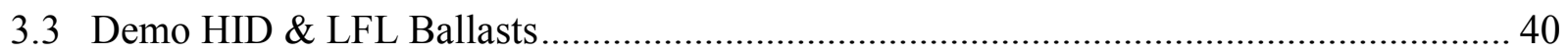

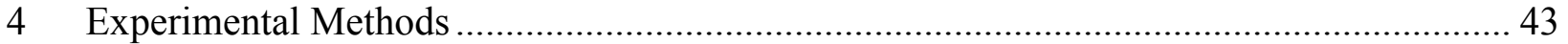

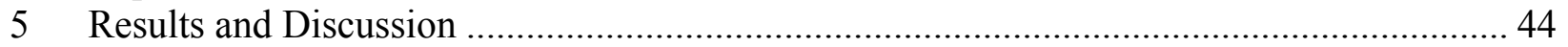

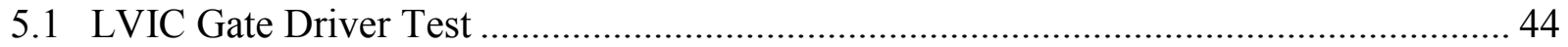

5.1.1 Auxiliary Circuit Test (Bias and Clock Generation Blocks) .................................. 44

5.1.2 Charge Pump-Based Low and High Side Power Supply Test.................................. 46

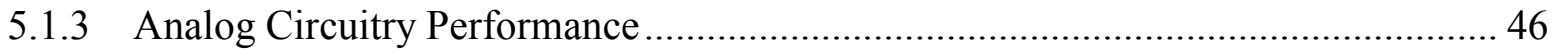

5.1.4 Digital Logic Test (LS and HS Communication): ................................................... 52

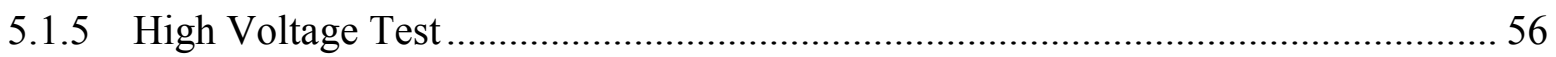

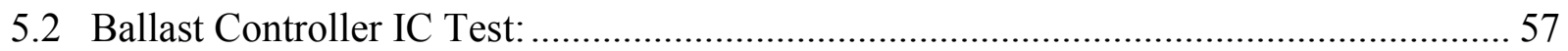

5.2.1 Auxiliary Circuit Test (Bias and Clock Generation Blocks) ................................. 57

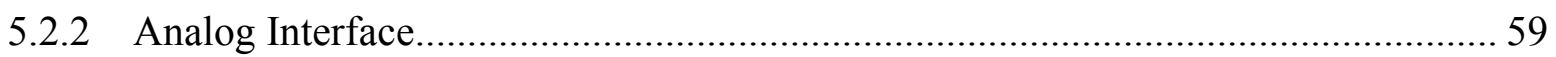

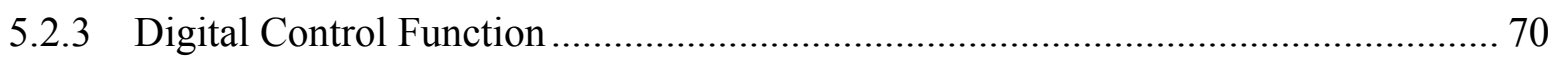

5.3 Ballast Controller and Gate Drive IC Performance in Demo Ballasts.............................. 75

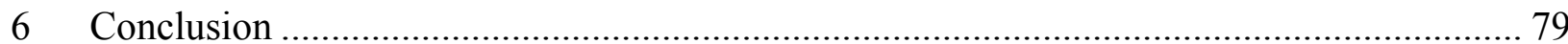

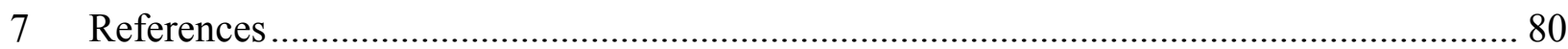

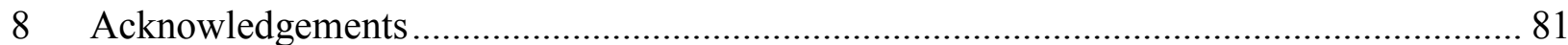

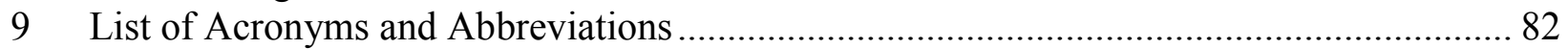




\section{LIST OF GRAPHICAL MATERIALS}

Figure 1-1. Block diagram of a universal control platform for high frequency electronic ballast

Figure 3-1. Dual-LVIC based gate drive concept applied to half-bridge ballast 5

Figure 3-2. Four operation states for LVIC based gate driver 5

Figure 3-3. Coupling capacitor current waveform for ZVS operation as a function of time 6

Figure 3-4. Schematic of discrete test circuit for dual LVIC gate driver 8

Figure 3-5. Control signals 9

Figure 3-6. Stead-state waveforms, ch1: HS gate drive; ch2: LS gate drive; ch3: inductor

current, 2A/div; ch4: midpoint voltage 9

Figure 3-7. HS \& LS supply voltages, ch1: HS supply voltage; ch2: LS gate drive; ch3: LS

$\begin{array}{ll}\text { supply voltage } & 10\end{array}$

Figure 3-8. Coupling capacitor currents, ch1: capacitor current, 0.5A/div; ch4: midpoint voltage. a) Falling transient, b) Rising transient $\quad 10$

Figure 3-9. Simplified block diagram of the custom IC 11

Figure 3-10. CAD layout of the primary nmos $\left(\mathrm{M}_{1} \& \mathrm{M}_{3}\right)$ and $\operatorname{pmos}\left(\mathrm{M}_{2} \& \mathrm{M}_{4}\right)$ switches 12

Figure 3-11. Signaling and handshaking current polarities in the LSIC and HSIC switches M1 $\& \mathrm{M} 2$

Figure 3-12. Schematic diagram of the bi-directional current detector for the nmos switch $\mathrm{M}_{1}$; A direct dual circuit was used for sensing bi-directional current in the pmos device $\mathrm{M}_{2}$

Figure 3-13. Schematic diagram of the fast current-mode comparator circuit. Feedback is used to maintain a low impedance input node for high-speed response with output buffers for sharp output edges.

Figure 3-14. Sate transition diagram for a) LSIC, and b) HSIC.

Figure 3-15. Microphotograph of custom IC fabricated in the AMS 0.8 $\mu \mathrm{m}$ CMOS process with core blocks labeled.

Figure 3-16. Corner simulation results of current detector for positive current

Figure 3-17. Corner simulation results of current detector for negative input current 17

Figure 3-18. Simulation results of low-side digital controller 18

Figure 3-19. Simulation results of high-side digital controller $\quad 18$

Figure 3-20. Simulation setup for driving half-bridge electronic ballast 19

Figure 3-21. System simulation results of LCC ballast: gate drive and resonant waveforms 19

Figure 3-22. System simulation of LCC ballast: capacitor current and comparator waveforms 20

Figure 3-23. Simplified HF electronic ballast configuration with LCC resonant tank 21

Figure 3-24. Bode plot of input impedance of a typical resonant tank 21

Figure 3-25. Direct digital phase control scheme with inductor current sensing 22

Figure 3-26. Phase control in a half-bridge LCC ballast using inductor current and midpoint voltage.

Figure 3-27. State machine of phase controller with inductor current sensing $\quad 25$

Figure 3-28. FPGA-based verification circuit for direct digital phase control 26

Figure 3-29. Steady-state waveforms of midpoint voltage and inductor current for different phase command, ch1: inductor current, 2A/div; ch2: midpoint voltage, 20V.div; 
a): $56.25^{\circ}$ commanded, $54.98^{\circ}$ measured; b) $33.75^{\circ}$ commanded, $32.18^{\circ}$ measured; c) $11.25^{\circ}$ commanded, $10.12^{\circ}$ measured.

27

27

Figure 3-31. Simplified block diagram of the ballast controller IC with two primary loops: 1) inner phase control and 2) outer lamp current regulation.

28

30

Figure 3-32. Block diagram of the ballast controller custom IC

Figure 3-33. Block diagram of the startup controller, which sweeps phase from $90^{\circ}$ toward $0^{\circ}$

Figure 3-34. Block diagram of the peak detector, which latches the peak lamp current in each switching cycle

Figure 3-35. Block diagram of the compensator block, which provides integral compensation to the lamp current regulation loop

Figure 3-36. Block diagram of the startup controller combined with the compensator for smooth transitions between operating modes

Figure 3-37. Analog ac buffer diagram

Figure 3-38. Block diagram for current-mode A/D conversion

Figure 3-39. Simplified conceptual diagram of the current-mode rectifier

Figure 3-40. First two MSBs of the asynchronous pipe-lined A/D converter

Figure 3-41. System diagram of FPGA-based prototype for ballast controller verification

Figure 3-42. Steady-state waveforms for HID ballast with resistive load, ch1: lamp voltage; ch2: lamp current; ch3: inductor current; ch4: midpoint voltage; a) load current $=3 \mathrm{~A}$ peak, load power $=350 \mathrm{~W} ; \mathrm{b}$ ) load current $=2 \mathrm{~A}$ peak, load power $=190 \mathrm{~W}$

Figure 3-43. Step responses for HID ballast with resistive load: load current $=2.5 \mathrm{~A} \rightarrow 2.8 \mathrm{~A}$ peak, ch1: lamp voltage; ch2: lamp current; ch3: inductor current; ch4: midpoint voltage

Figure 3-44. Start-up waveforms with resistive load, ch2: output voltage; ch3: inductor current

Figure 3-45. Photograph of the ballast controller, measuring $4.2 \mathrm{~mm} \times 3.2 \mathrm{~mm}$

Figure 3-46. Simulation results for ac buffer (input ac voltage: $500 \mathrm{mV}$; output current: $11.85 \mu \mathrm{A})$

Figure 3-47. Simulation results for I-mode rectifier

Figure 3-48. Simulation result for sample and hole circuit

Figure 3-49. Simulation result for A/D converter by sweeping dc input current from $12.5 \mu \mathrm{A}$ to $37.5 \mu \mathrm{A}$

Figure 3-50. Prototype ICs: (a) gate driver IC in 28-pin package; (b) ballast controller IC in 68-pin package.

Figure 5-2. Current detector output current, a) measured, b) calculated with a factor of 500

Figure 5-3. Experimental results demonstrating current scaling and detection operations for $\pm 100 \mathrm{~mA}$ injection for a) triangle waveform, and b) square waveform; ch1: injection current to cap pin $50 \mathrm{~mA} / \mathrm{dov}$, ch2: current detector output $50 \mu \mathrm{A} / \mathrm{div}$

Figure 5-4. Experimental results demonstrating current comparator operation with a $+68 \mathrm{~mA}$ threshold, ch1: injection current $20 \mathrm{~mA} / \mathrm{div}$, ch2: current comparator output

Figure 5-5. On-resistance of n-channel MOSFET 
Figure 5-6. On-resistance of p-channel MOSFET

Figure 5-7. Experimental results showing correct logic control operation depending on correct detection of signaling and handshaking currents; a) HSIC failed to detect rising transition; b) Unreliable handshaking detection of LSIC; c) Occurrence of correct handshaking detection of LSIC increases with decrease of supply voltage; d) Correct operation with 10.5V LS supply voltage and 14V HS supply voltage; ch1: LS gate drive; ch2: LS cap drive; ch3: HS cap drive; ch4: HS gate drive; All with 10V/div.

Figure 5-8. Operation area of LS and HS supply voltage, inside purple area LS and HS ICs can communicate each other

Figure 5-9. Detailed waveforms showing communication between LS and HS ICs with 14V LS and HS supply voltage: a) a proper falling transition, b) a proper rising transition, c) missed LS gate drive in falling transition

Figure 5-10. High Voltage Test Setup for demonstration of dual LVIC operation

Figure 5-11. Experimental results showing the two sides of the coupling capacitor pin (LS and HS) during signaling associated with the rising transition

Figure 5-12. Internal A/D test with $44 \mathrm{kHz}$ input showing a correct clock frequency generated based on adc_sel settings; (a) 32x fs with adc_sel $=0$; (b) $16 \mathrm{x}$ fs with adc_sel $=1$

Figure 5-13. Zero crossing detection results demonstrating effect of dc-injection cancellation: duty ratio keeps 0.5 over wide range of input magnitude and frequency: (a) $\mathrm{V}_{\text {in }}=$ $0.4 \mathrm{~V}, 40 \mathrm{kHz}$; (b) $\mathrm{V}_{\text {in }}=0.1 \mathrm{~V}, 40 \mathrm{kHz}$; (c) $\mathrm{V}_{\text {in }}=0.4 \mathrm{~V}, 300 \mathrm{kHz}$; (d) $\mathrm{V}_{\text {in }}=0.1 \mathrm{~V}$, $300 \mathrm{kHz}$

Figure 5-14. Output current waveforms from I-mode rectifier showing a) dc-offset caused asymmetric peaks, b) symmetric peaks with $\sim 1.7 \mu \mathrm{A}$ injected into positive input

Figure 5-15. Plots from logic analyzer illustrating the effect of dc-offset cancellation, a) without dc-bias resistor connected, b) with resistor connected (the waveforms were inverted)

Figure 5-16. Plots from logic analyzer showing the linearity between the input peak voltage and output peak current of A/D circuitry, a "flat spot" was noticed around 55\% of full scale input peak voltage $(0.58 \mathrm{Vpk} / 1.05 \mathrm{Vpk})$

Figure 5-17. Average digital output v.s. actual current (emulated by DC power supply) with an external A/D clock at $10 \mathrm{kHz}$

Figure 5-18. Average digital output vs. actual current with an external A/D clock at $167 \mathrm{kHz}$

Figure 5-19. Average digital output vs. actual current with internal A/D clock at $2.7 \mathrm{MHz}$ and 300W output power

Figure 5-20. Number of digital spikes outside \pm 2 -bit window w/ an external A/D clock at $10 \mathrm{kHz}$

Figure 5-21. Number of digital spikes outside \pm 2 -bit window w/ an external A/D clock at $167 \mathrm{kHz}$

Figure 5-22. Number of digital spikes outside \pm 2 -bit window with an internal A/D clock at 2.7 MHz, 300W output power

Figure 5-23. External comparator providing over voltage signal to voltage channel to bypass spike caused problem. The output of the comparator keeps low $(\sim 0.2 \mathrm{~V})$ at normal operation; it output $\sim 4.9 \mathrm{~V}$ at over voltage protection mode, which cause lamp voltage channel trigger on-chip over-voltage protection block. 
Figure 5-24. Steady state waveforms at DCO mode, with $300 \mathrm{~V}$ dc bus voltage and 16x AD sample rate. Ch1: lamp current; $\mathrm{Ch} 2$ : inductor current; Ch 3: lamp voltage; $\mathrm{Ch}$ 4: midpoint voltage

Figure 5-25. Steady state waveforms at different current command: a) 1.28A, b) 2.56A, c) 3.84A, and d) 4.48A; Ch1: lamp current; Ch 2: inductor current; Ch 3: lamp voltage; $\mathrm{Ch} 4$ : midpoint voltage

Figure 5-26. Steady state operation of $400 \mathrm{~W}$ HID lamp with $300 \mathrm{~V}$ DC bus voltage, 4.8A lamp peak current, $136 \mathrm{kHz}$ operation frequency, $58^{\circ}$ measured phase and $61^{\circ}$ phase command

Figure 5-27. Over voltage protection showing a repeated ignition attempts of HID lamp until it ignited below preset $3.5 \mathrm{kV}$ protection level

Figure 5-28. Resistor load start-up waveforms with 2.56A current command, ch1: output current, ch2: inductor current, ch3: load voltage, ch4: midpoint voltage

Figure 5-29. HID lamp ignition sequence with operation modes labeled. Dc bus voltage $=300 \mathrm{~V}$, current commend $=4.8 \mathrm{~A}$

Figure 5-30. HID lamp ignition dynamics in the first few cycle

Figure 5-31. Demo ballast performance for resistor load at near rated power: (a) start up sequence with expected operation modes; (b) steady state operation with $1 \%$ regulation error; ch1: lamp current, $200 \mathrm{~mA} / \mathrm{div}$; ch2: midpoint voltage, $200 \mathrm{~V} / \mathrm{div}$; ch3: sensed inductor current from a CT loaded by $20 \Omega, 0.5 \mathrm{~V} / \mathrm{div}$; ch4: LS cap voltage, $10 \mathrm{~V} / \mathrm{div}$

Figure 5-32. Photograph demonstrating steady-state operation of demo ballast driving a $32 \mathrm{~W}$ T8 energy efficient LFL lamp

Figure 5-33. Experimental results showing performance of demo ballast driving a 32W T8 LFL lamp at near rated power: (a) start up sequence with expected operation modes; (b) steady state operation with 5\% regulation error; ch1: lamp current, $200 \mathrm{~mA} /$ div; ch2: midpoint voltage, $200 \mathrm{~V} /$ div; ch3: sensed inductor current from a CT loaded by $20 \Omega, 0.5 \mathrm{~V} / \mathrm{div}$; ch4: LS cap voltage, $10 \mathrm{~V} / \mathrm{div}$

Figure 5-34. Experimental results demonstrating robust operation of the dual LVIC gate drive prototype IC: a) falling transition; b) rising transition; ch1: lamp current, 200mA/div; ch2: midpoint voltage, 200V/div; ch3: sensed inductor current from a CT loaded by $20 \Omega, 0.5 \mathrm{~V} / \mathrm{div}$; ch 4 : LS cap voltage, $10 \mathrm{~V} / \mathrm{div}$

Figure 6-1. Potential pin assignment of a) LVIC gate drive and b) Ballast controller IC 


\section{INTRODUCTION}

The trend to replace electromagnetic ballasts with electronic ballasts for energy efficient lighting, such as linear fluorescent lamps (LFL), compact fluorescent lamps (CFL), and high intensity discharge lamps (HID) is accelerating. This is mainly driven by huge energy saving benefits due to a) much higher ballast efficiency; b) higher efficacy for lamps running at higher frequency; c) longer lamp lifetime; and d) energy saving features such as dimming capability, especially when combined with sensors and communication networks in buildings.

However, the current controllers for electronics ballast products are either largely based on discrete circuitry and/or point of solutions for specific type of lamps. In this report, a new approach utilizing IC-based controls is developed and documented that will increase penetration of highly efficient electronic ballast and energy efficient lighting with lower cost, sophisticated control features that drive the lamps in their optimum conditions and provide additional capabilities such as dimming, remote monitoring/control and communication.

The developed IC-based controls could also be regarded as a universal high frequency ballast control platform, which consists of gate drive IC, ballast controller IC and lamp controller IC, as shown in Figure 1-1. The gate drive IC provides sufficient gate driving capability to turn on/off both ground-referenced and floating power switches with a proper timing to avoid simultaneous conduction (anti-shoot through protection) and support zero-voltage switching (ZVS) operation for reduced losses. The ballast controller IC fully integrates the most common control features, including programmable ignition and transition to normal operation, ac lamp current regulation, over-voltage protection, dimming control, and self tuning digital phase control that allows ballast controller IC to be used in a wide range of lamps. It provides a simple digital interface for communication with the lamp controller IC. The lamp controller IC implements more lampspecific control functions, such as start-up mode control, hot re-strike protection, acoustic resonance detection and avoidance, spread-spectrum control and lamp diagnostics etc., and network-enable interface for the networked lighting system.

Many techniques are available for achieving the control platform proposed above. The pulse transformer, for example, is frequently used for isolation and can be used as a floating gate drive. However, in high frequency switching converters operating from hundreds of $\mathrm{kHz}$ to $\mathrm{MHz}$, the advanced magnetics and additional passive components required for high efficiency operation become prohibitively expensive. High voltage IC (HVIC) technologies are also frequently used for gate drivers due to their ability to directly drive the floating high side through high voltage silicon processes for high voltage isolation. However, at high frequencies they usually suffer from significant heating due to static and dynamic losses of the level-shifting circuitry and dynamic losses of the high side isolation well capacitance. New technologies such as silicon-oninsulator (SOI) promise to offer high voltage isolation with reduced parasitic capacitance, but require custom processing and high cost.

Traditional ballast controllers are built with passive components and discrete opamps. Some analog IC based ballast controllers are available recently that have greatly reduced the size and cost. The rapid development in digital technology makes the digital controller have many 
advantages over its analog counterpart, including lower sensitivity to parameter and temperature variations, fewer external components, and smaller size. It can also utilize the fast advances in digital CAD and silicon processing for full design automation, re-programmability, and low cost hardware implementation. By targeting standard sub-micron digital processes, the hardwarebased digital controller is not only cost effective, but also provides fine time resolution (down to sub nanosecond), allowing accurate control even in the $\mathrm{MHz}$ switching frequency range. With these benefits, a digital ballast controller provides the possibility to further reduce the cost, and incorporate more advanced and intelligent control functions as lamp protection, EMI reduction, and remote control and monitoring.

The implementation of the lamp controller could be any off-the-shelf low-cost ICs, such as micro-controller or programmable logic (CPLD or FPGA), since the lamp specialized control functions are implemented. These control functions require only relatively slow time constants (10's ms or greater) and are ideal for implementation in standard digital parts, allowing us to leverage the aggressive pricing found in the high volume market place.

In this report, a new control IC chip set for the ballast control platform, including dual low voltage IC (LVIC) gate drive and ballast controller IC with digital control, will be presented. The concept development, IC design details, demo ballast operation, and experimental verifications will be fully covered.

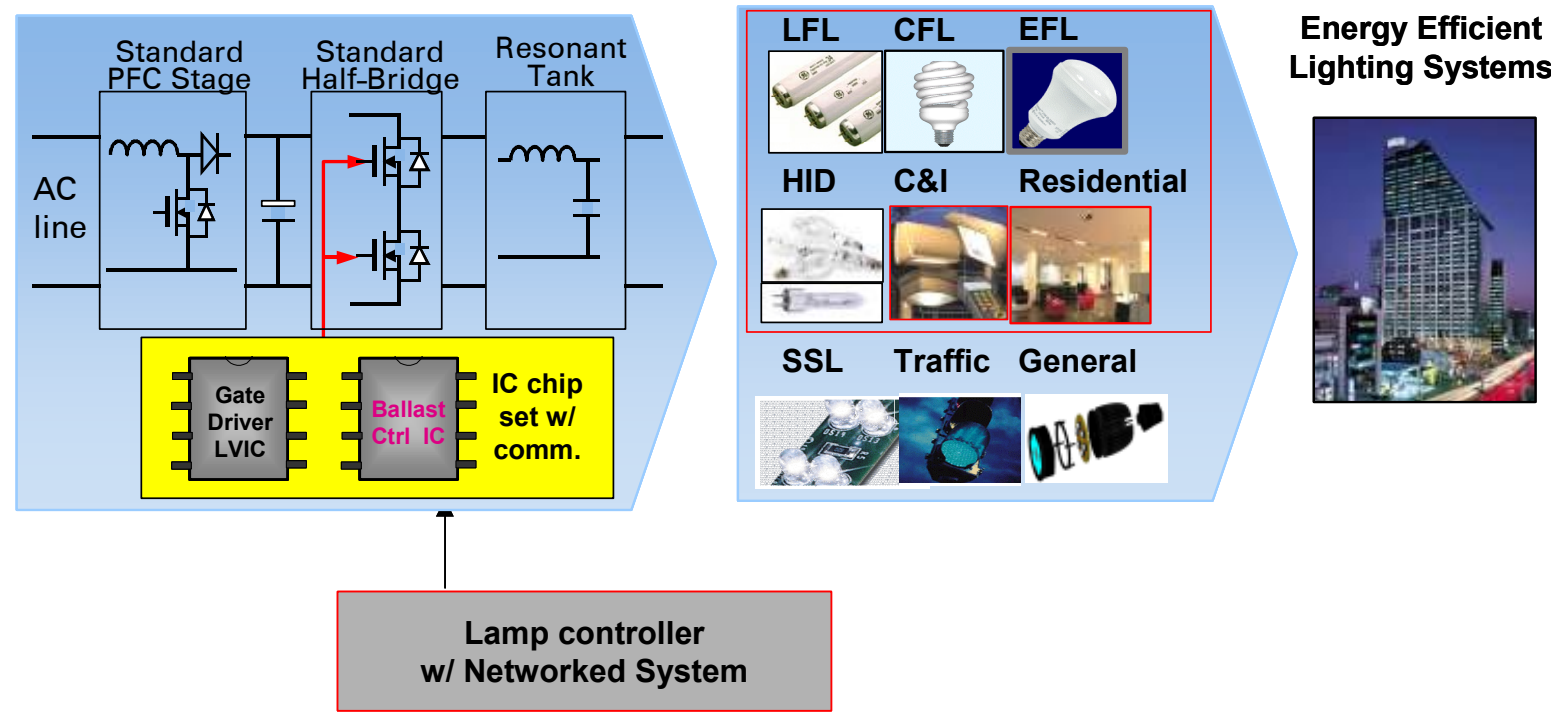

Figure 1-1. Block diagram of a universal control platform for high frequency electronic ballast 


\section{EXECUTIVE SUMMARY}

A new IC-based control platform for energy-efficient lighting has been developed and demonstrated through prototype ICs and demo ballasts driving high intensity discharge (HID) and linear fluorescent lamps (LFL). The developed prototype IC chip set includes a low cost dual LVIC gate drive providing low and high side gate drive to power switches and a fully integrated ballast controller IC with direct digital phase control. The benefits of the developed approach include reduced system cost, reduced ballast size, universal application to a wide range of lamp technologies, and potential networking capability for networked lighting system.

The developed dual low voltage IC (LVIC) gate drive prototype provides a low-cost alternative to existing high voltage IC (HVIC) gate drive products. It utilizes a single coupling capacitor to accomplish three functions: 1) high voltage isolation, 2) high and low side power supply, and 3) signaling to the high side for on/off control. Integrated switches are used on each IC to provide active current steering for charge-pump power supply operation and current sensing for signaling detection. The dual LVIC approach provides the benefits of minimal external component count, built-in power supply, low-cost IC fabrication, adaptive dead time control (when operating under ZVS), and high frequency operation.

The developed ballast controller IC prototype utilizes the advantages of digital technology to reduce the cost, incorporate more advanced and intelligent features and reduce dependence on process and temperature. The core of the controller IC is based on a two-loop control with a direct digital phase control inner loop and an ac lamp current regulation outer loop. The inner loop provides near cycle-by-cycle phase regulation with the advantages of self-tuning to the resonant frequency, reduced sensitivity, inherent zero-voltage switching and simplified dynamics for current regulation. The outer loop provides ac lamp current sensing and regulation for lamp ignition and dimming control. Buffered ac sensing of phase, lamp current and voltage facilitate fast detection, improved efficiency and lower cost by removing external rectifying diodes and filtering. In addition, the IC provides a simple digital interface for communication.

The developed demo ballast platform verified the performance of the developed IC chip set driving HID and LFL lamps. The success of IC-based controls will increase penetration of highly efficient electronic ballast and energy efficient lighting products with lower cost and sophisticated control features and drive the lamps in their optimum conditions with additional capabilities such as dimming, remote monitoring/control and communication. 


\section{TECHNICAL PROGRESS}

The program is focused on the development and demonstration of control IC chip set for the ballast control platform. Two ICs, one a low cost dual-LV gate driver IC and the other a fully integrated ballast digital controller IC, were developed and verified through demo ballast for HID and LFL lamps. In the following sections, new concepts and design details for the development of dual LVIC gate drive and ballast controller IC with direct digital phase control are presented.

\subsection{Dual Low Voltage IC (LVIC) Based Gate Drive}

Half-bridge switching network is a typical configuration widely used in electronic ballasts. The gate drive is required to provide sufficient driving capability to power switches with a proper timing requirement, to deliver a floating supply to the high side drive, and to create a proper dead-time between the power switches to avoid simultaneous conduction and support zerovoltage switching (ZVS) operation for reduced switching losses. High voltage isolation is usually required, as is typical in half and full-bridge configurations where both ground and floating referenced power switches are controlled on/off by the gate driver circuitry. A dual LVIC based low and high side gate driving approach is proposed and the IC solution of the gate driver using low voltage silicon process is implemented.

\subsubsection{Dual LVIC Gate Drive Concept}

Figure 3-1 shows a simplified diagram for the dual LVIC gate drive concept applied to the typical half-bridge high frequency electronic ballasts. A capacitor is employed to interface the high side and low side drivers. A single-pole double-throw (SPDT) switch is used in both low side (S1) and high side (S3) circuits (ICs) to switch the coupling capacitor between Vdd and its referred ground to position the switches for charge-pump operation and to generate the signaling currents. Another SPDT switch (S2 \& S4) is used to drive the gate of each power device. 


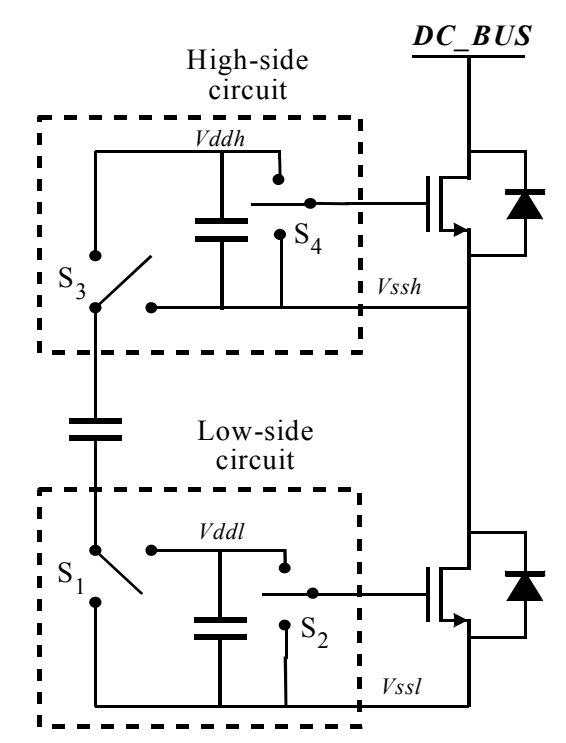

Figure 3-1. Dual-LVIC based gate drive concept applied to half-bridge ballast

The operation of the gate driver can be described in four states for the half-bridge configuration based on the status of the midpoint voltage $V_{m}$ as shown in Figure 3-2. The switch timing and resulting coupling capacitor current is key to understanding system operation. The capacitor current pulses associated with each state are labeled in Figure 3-2 with numbers, 1-6, and Figure 3-3 shows the capacitor current as a function of time for each of the six current pulses together with the switching instances and operating states under ZVS conditions.

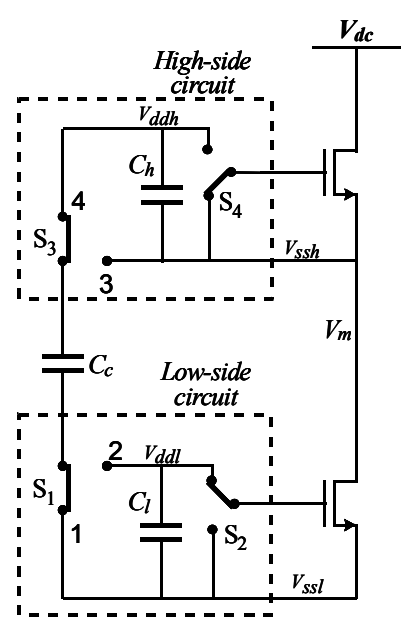

(a) Low side on

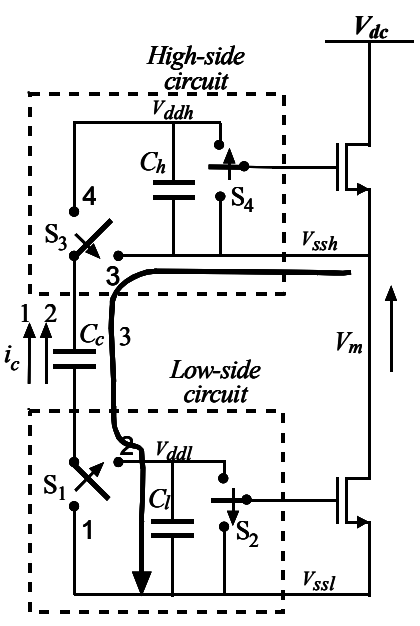

(b) Rising transition

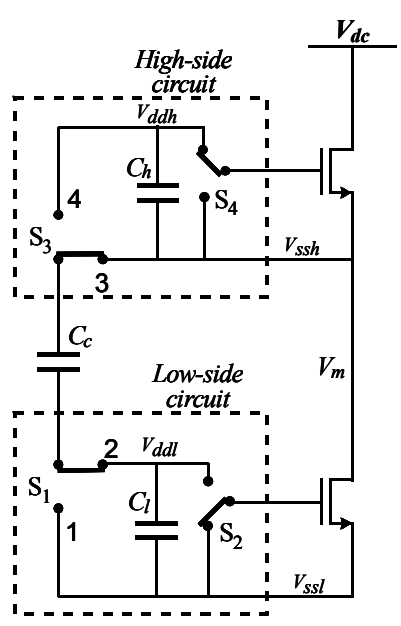

(c) High side on

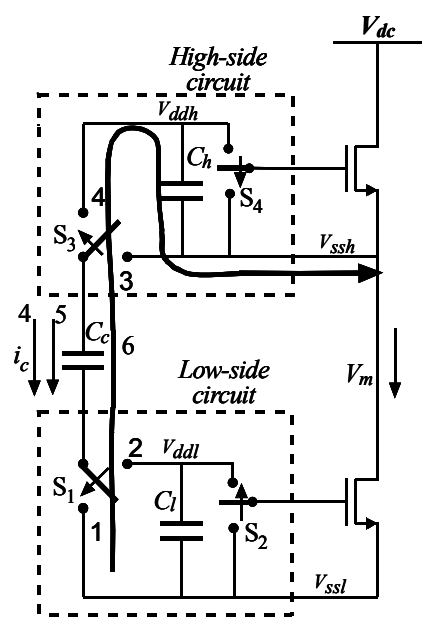

(d) Falling transition

Figure 3-2. Four operation states for LVIC based gate driver 


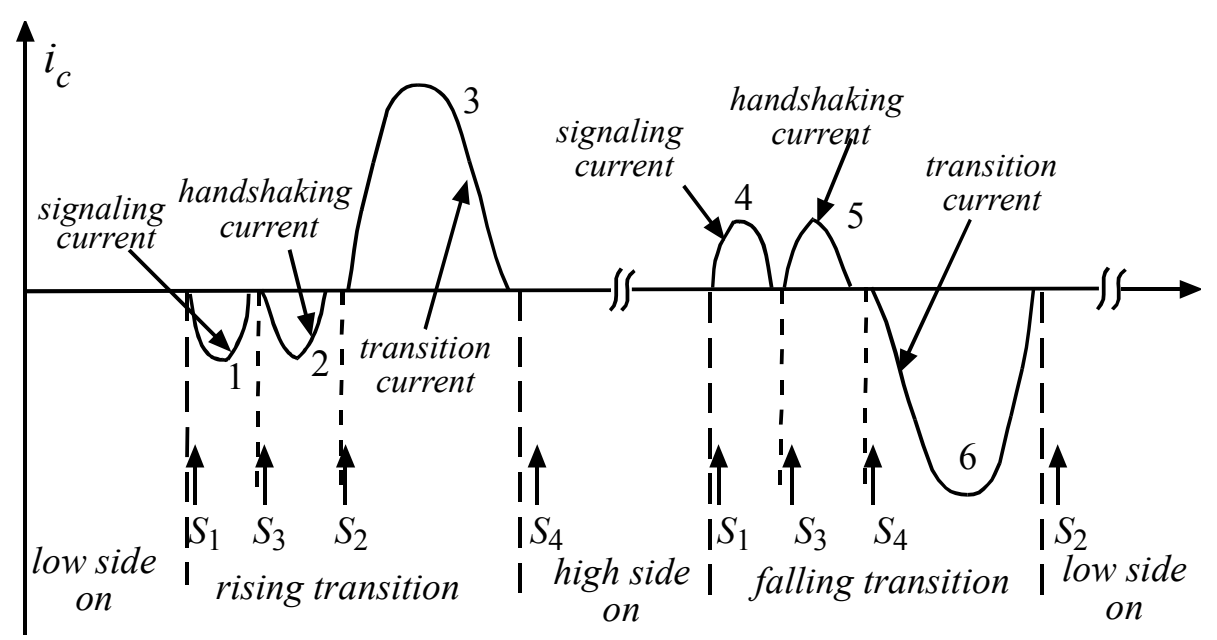

Figure 3-3. Coupling capacitor current waveform for ZVS operation as a function of time

\section{Low Side On State}

Under this state, the low side gate is on with S1 tied to Vssl, and the high side gate is off with S3 tied to Vddh, as illustrated in Figure 3-2(a). As the midpoint voltage Vm is zero (constant), no current flows through the coupling capacitor Cc.

\section{Rising Transition State}

This state refers to the rising transition of Vm. Following the command from the controller, $\mathrm{S} 1$ is first switched from Vssl to Vddl as shown in Figure 3-2(b), which generates a pulse current (defined as "signaling current") pumped up to the high side through Cc, as shown in Figure 3-3. The high side controller, after sensing this signaling current, switches S3 from Vddh to Vssh to generate another pulse (defined as "handshaking current") that also flows through Cc. Following detection of the handshaking current, the low side controller switches S2 to Vssl to turn off the low side gate immediately (or enters a fault condition if no handshaking current is received). In applications where the half-bridge is used to drive a resonant tank under ZVS, the midpoint voltage Vm will then increase, charging the coupling capacitor Cc as well as the low side energy storage capacitor $\mathrm{Cl}$. As a result, a large transition current flows through the coupling capacitor, which is also shown in Figure 3-3. After a fixed deadtime, the high side controller turns on the high side gate with soft switching by switching S4 from Vssh to Vddh. If the converter does not operate under ZVS, the high side gate will be forced to turn on after a fixed deadtime with hard switching, which will create a transition current with higher amplitude and shorter time through the coupling capacitor.

\section{High Side On State}


This state comes right after the rising transition state. It is similar to the low side on state except the high side gate is on with S1 tied to Vddl and S3 tied to Vssh as shown in Figure 3-2(c). Vm is tied to the dc bus voltage and no current flows through $\mathrm{Cc}$.

\section{Falling Transition State}

This state refers to the falling transition of $\mathrm{Vm}$. At the end of the switching period, the low side controller switches S1 from Vddl to Vssl according to the control command, generating a signaling current, which is opposite to the one in the rising transition. In a similar way to the rising transition, the high side detects this pulse and switches S3 from Vssh to Vddh, resulting in the handshaking current. The high side gate turns off, triggering the falling transition of $\mathrm{Vm}$ if the converter operates under ZVS. The energy stored in the coupling capacitor is then transferred to the high side energy storage capacitor $\mathrm{Ch}$. The low side controller detects the handshaking current and waits for a deadtime, then turns on the low side gate to lead the system into the lowside-on state. If the converter does not operate under ZVS, the low side turn-on will be hard switching, which is similar to the high side turn-on in hard switching.

Figure 3-3 illustrates the waveform of the current flowing through the coupling capacitor, which is fundamental to the operation of the dual LVIC gate driver. The signaling and handshaking currents are used for communication between the low and high side ICs, and the transition currents supply energy to both ICs. It can also be seen that the coupling capacitor blocks dc bus voltage (up to several hundred volts) when the high side gate is on. Hence, this coupling capacitor implements three functions: high voltage isolation, communication interface between the low and high side ICs, and charge pump power supply. Extensions for adaptive dead-time control can be achieved using a slight modification to the operating mode sequencing. At the conclusion of the transition operating modes b) and d), the respective device turn-on is set by detection of the end of the transition current in the coupling capacitor rather than after a fixed dead time. During intervals of hard switching, such as startup or step load changes, the circuit could revert to a maximum dead time when the end-of-transition detection does not occur within a specified time. This simple modification achieves maximum efficiency and removes the dependence on timing parameters within an IC that will vary with process and temperature. This feature was not included in the prototype IC in order to first validate the core operating concepts and high-risk components.

\subsubsection{Discrete Verification}

A discrete test-bed is built for experimental verification of the key switching concepts described in Figure 3-2 and the resulting waveform in Figure 3-3. The primary objective of the discrete circuitry is to examine the charge-pump operation and the characteristics of the signaling and handshaking currents, which are critical for the on-chip current detector and the control logic design.

The key switching functions are implemented with discrete low voltage MOSFETs driven by optocouplers for high voltage isolation. These discrete switches emulate the on-chip SPDT 
switches. A separate signal generator built with flip-flops and multiplexers is used to provide proper signal sequencing to the discrete switches to drive the coupling capacitor and the power switches. Figure 3-4 outlines the key functional blocks for the testing circuitry: signal generator, optocouplers, low and high side LV SPDT switches, and a half-bridge resonant inverter (used as a representative application). The control signals for the switches are plotted in Figure 3-5. The purpose of the optocouplers for the low side switches is to match the delay to each discrete switch. The optocouplers are only an artifact of the discrete testing platform, and are not required for the IC solution where the switch control signals will be generated on chip. Two resistors are placed between the dc bus and the high and low side power supply capacitors to provide proper bias voltage for IC startup. During normal operation, their effects are negligible.

Half-bridge 400W HID ballast with resistive load is used in the test-bed. The power MOSFETs are chosen as 500V/20A devices STW20NM50FD and the gate charge is 38nC for hard switching. The coupling capacitor is chosen to be $300 \mathrm{pF}$ based on the considerations of 1) large enough to provide sufficient power to the two ICs and 2) small enough to avoid affecting ZVS operation and excessive transition currents in the ICs. The large power switches are chosen intentionally to test the gate driving capability of this driver for the worst-case operation. For lower power applications with smaller power switches, the smaller coupling capacitor can be selected, resulting in a lower magnitude of the capacitor current and smaller on-chip switches. The low side and high side supply capacitors are $1 \mu \mathrm{F}$ and clamped by $12 \mathrm{~V}$ zener diodes.

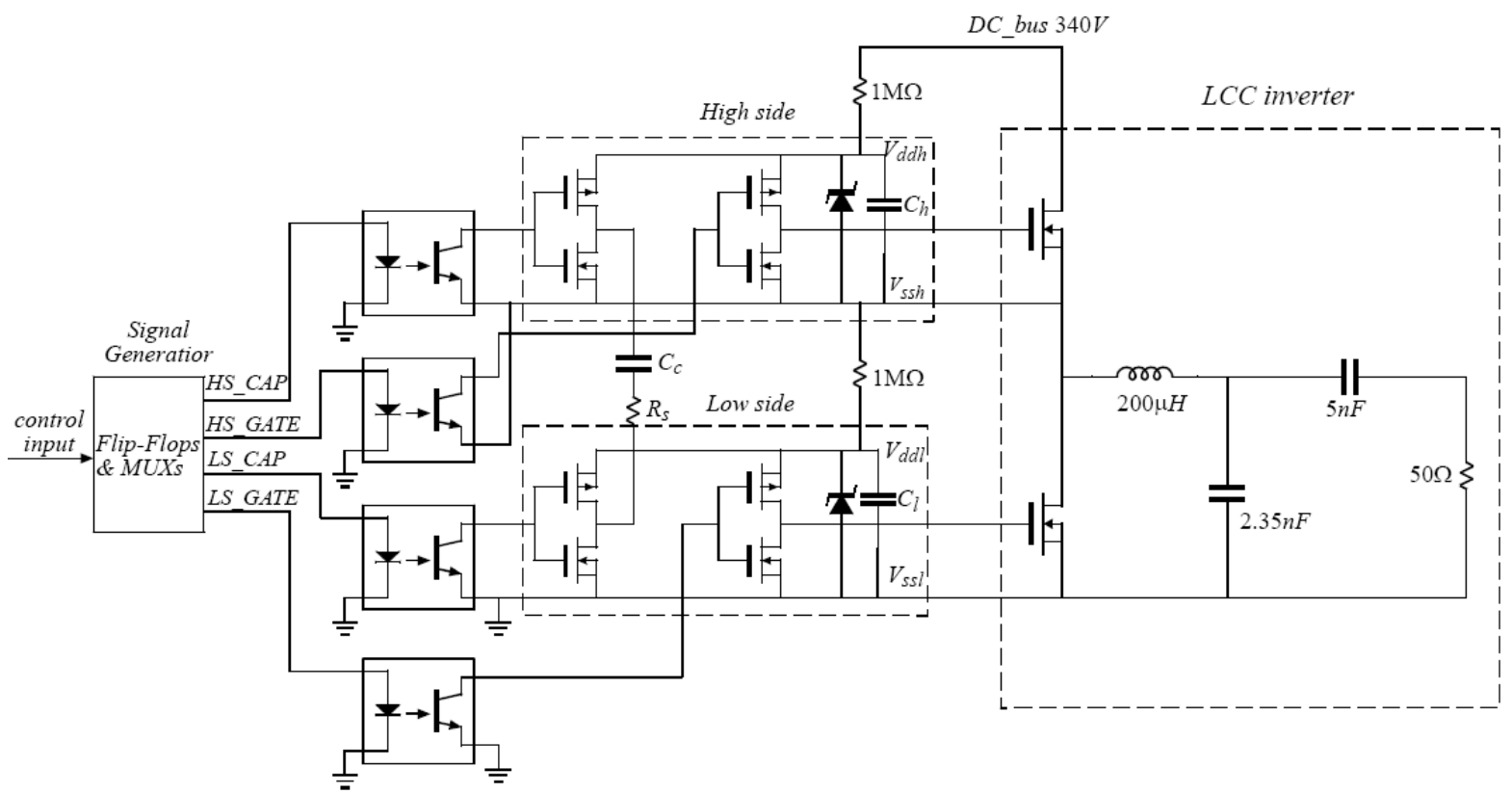

Figure 3-4. Schematic of discrete test circuit for dual LVIC gate driver 


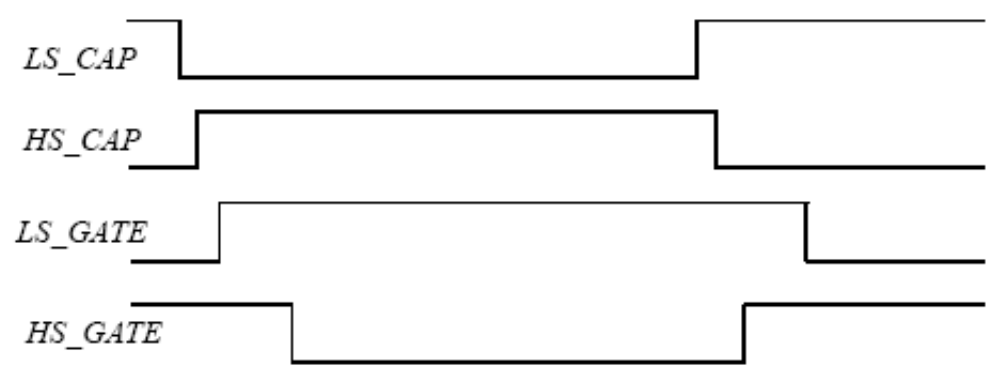

Figure 3-5. Control signals

Figure 3-6 presents the steady-state waveforms with the switching frequency of $170 \mathrm{kHz}$ and $34 \mathrm{~W}$ input power. It can be seen that the system works properly with zero-voltage switching. The high side and low side supply voltage waveforms are shown in Figure 3-7, which demonstrates that sufficient energy is passed through the coupling capacitor in each cycle to drive the gates of the power switches because the supply voltages are nearly constant. The ripple in the high side supply voltage appears to be coupled in through the high voltage differential probe used in this measurement (the probe dc bias varies with the midpoint voltage swing). The currents flowing through the coupling capacitor for falling and rising transitions are shown in Figure 3-8 (a) and (b) sensed by a $10 \Omega$ resistor Rs. They are in agreement with the theoretical results in Figure 3-3. The signaling and handshaking current is about 200mA with width of 50ns $\sim 100 \mathrm{~ns}$, and the transition current is about $2 \mathrm{~A}$. This data can be used to design a fully integrated LVIC solution, which will be discussed in the next section.

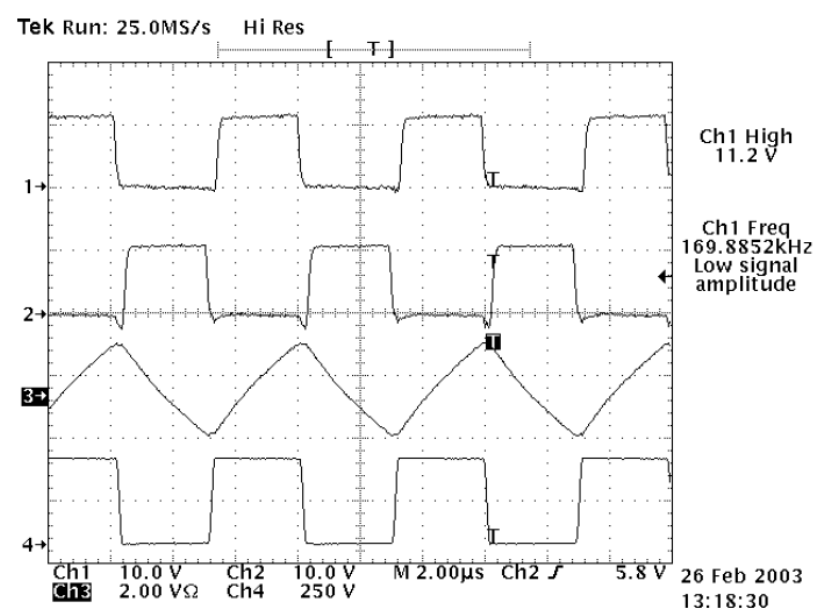

Figure 3-6. Stead-state waveforms, ch1: HS gate drive; ch2: LS gate drive; ch3: inductor current, 2A/div; ch4: midpoint voltage 


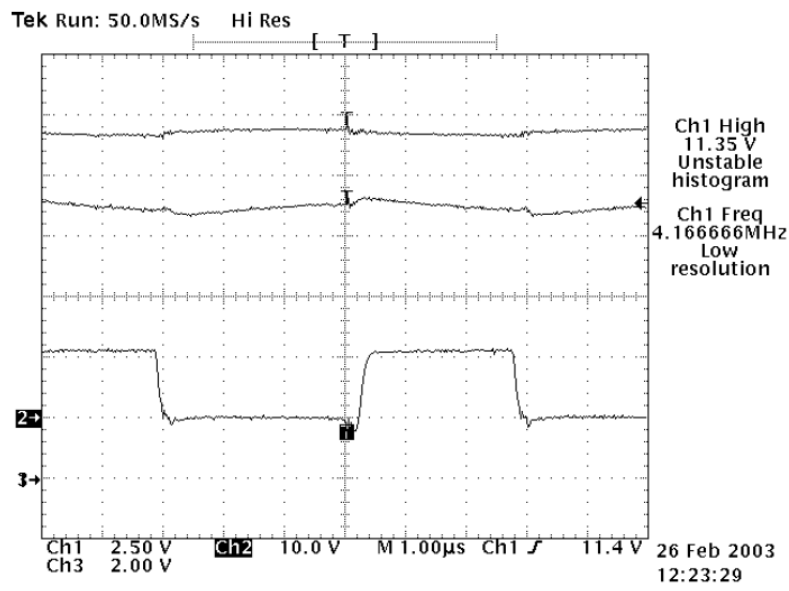

Figure 3-7. HS \& LS supply voltages, ch1: HS supply voltage; ch2: LS gate drive; ch3: LS supply voltage

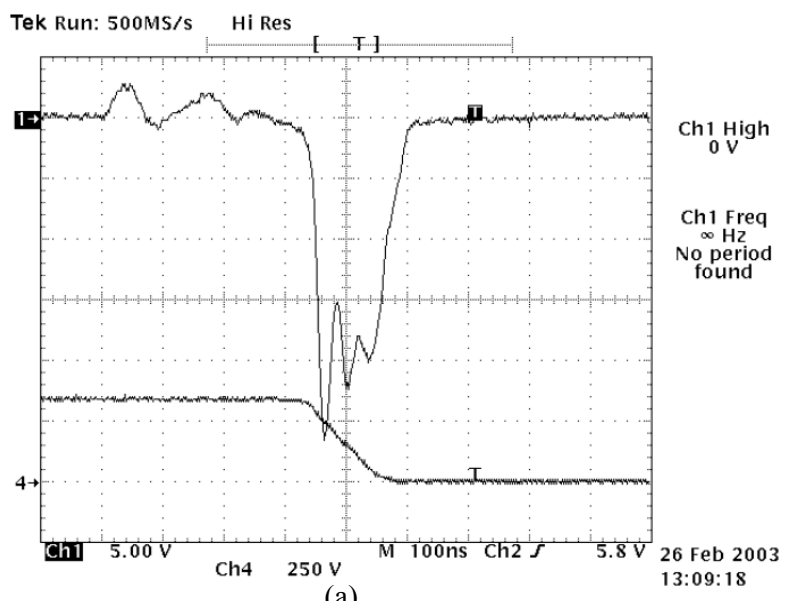

(a)

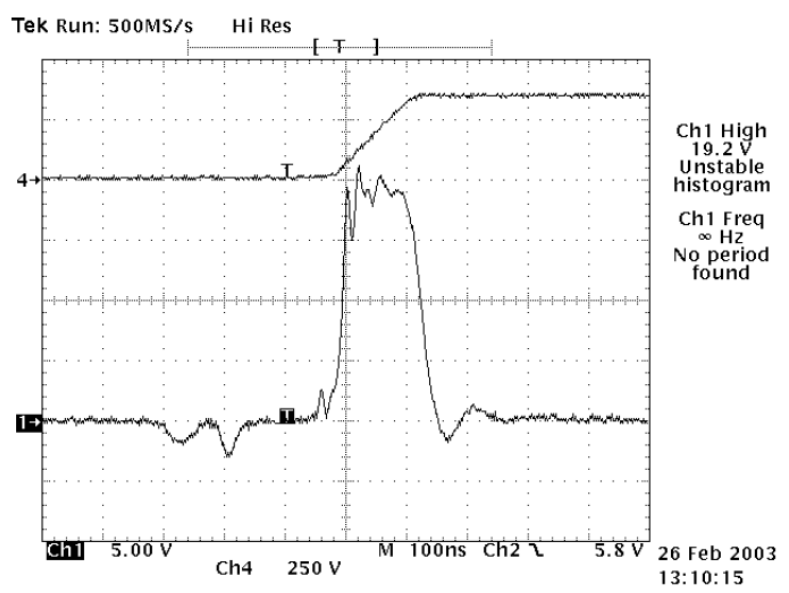

(b)

Figure 3-8. Coupling capacitor currents, ch1: capacitor current, $0.5 \mathrm{~A} / \mathrm{div}$; ch4: midpoint voltage. a) Falling transient, b) Rising transient 


\subsubsection{Gate Driver Custom IC Design}

A simplified block diagram of the custom IC is shown in Figure 3-9. The high and low side gate drivers use identical hardware with a single selection pin to adjust digital state-machine selection and threshold parameters for proper operation. The core blocks are the four main switches M1 and M2 (implementing S1 and S3 in Figure 3-1) and M3 and M4 (implementing S2 and S4 in Figure 3-11), bi-directional current mode detectors and comparators, and digital control logic. Additional supervisory functions and a voltage regulator are also included on the IC. The digital control logic performs state-machine operation to sequence operation states a) through d) and recognizes reset and start-up commands from voltage regulator. The CMOS switches are only required to withstand the gate driving voltage, typically between $8 \mathrm{~V}$ and $15 \mathrm{~V}$. This allows use of standard CMOS technologies to leverage high-volume processing for low-cost implementation, which is in contrast to the HVIC approach where the silicon must withstand up to $600 \mathrm{~V}$ and requires custom processing techniques. Each of the core IC blocks is described in more detail below.

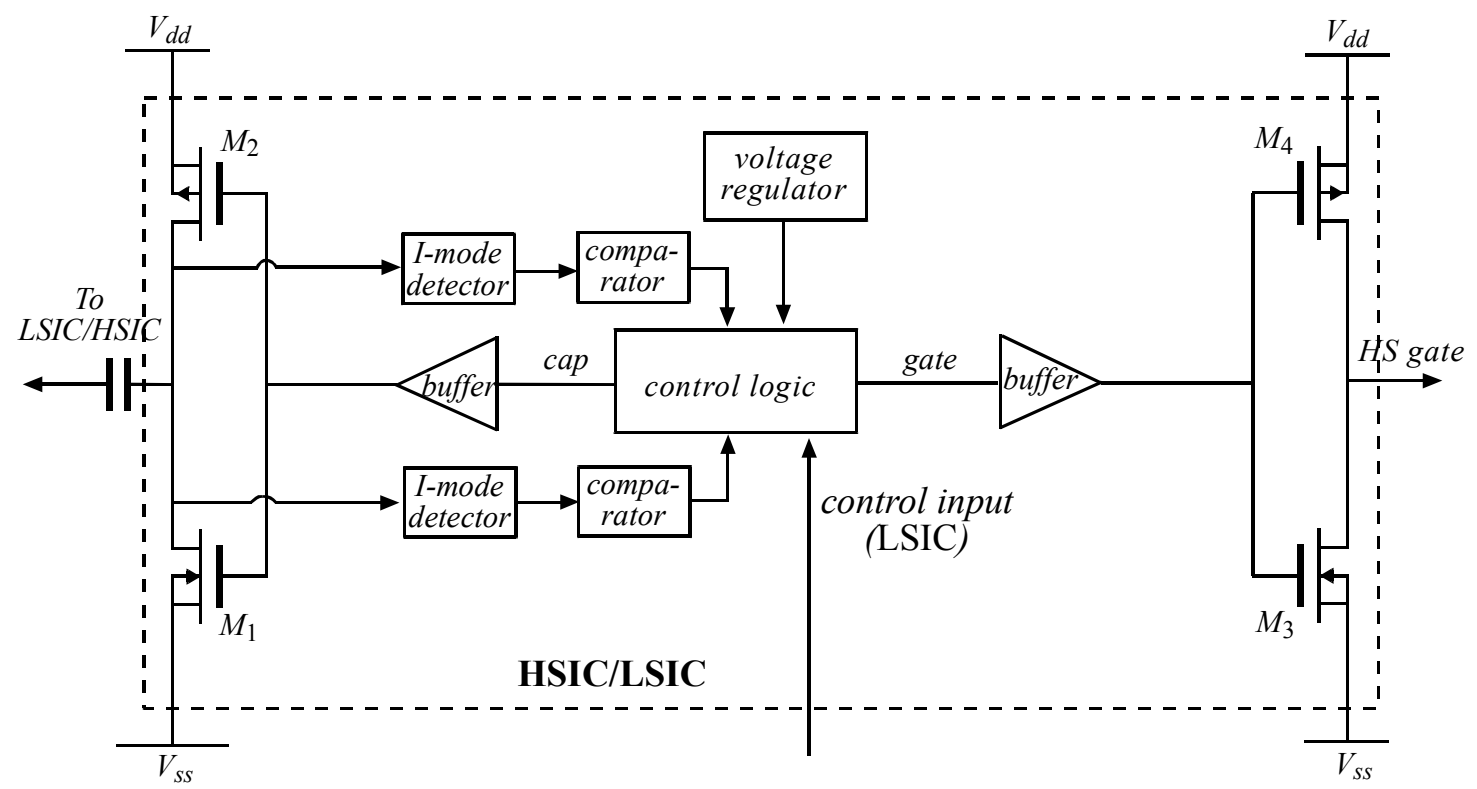

Figure 3-9. Simplified block diagram of the custom IC

\section{CMOS Switches}

The CMOS switches illustrated in Figure 3-9 (M1-M4) are used to drive the power switch and the coupling capacitor, with the layout shown in Figure 3-10. As these switches operate with high voltage $(8 \mathrm{~V} \sim 15 \mathrm{~V})$, they are implemented with mid-oxide DMOS technology. The sizes of these switches are chosen based on the requirements for gate driving, capacitor switching, and current conduction. The gate drive switches M3 and M4 must provide sufficient driving 
capability for fast switching of the power gates. The capacitor drive switches M1 and M2 determine the on-resistances and parasitic capacitances of these devices, which affect the switching speed as well as the magnitude and width of the signaling and handshaking currents. Due to similar requirements for both sets of switches, we chose to use the same nmos/pmos pair for gate drive and capacitor drive. A primary challenge in the layout is that in both functions (gate and capacitor drive), significant current flows in both directions. This creates concerns for system noise and body diode conduction effects on the remaining mixed-signal circuitry in the IC. For this design, the nmos is sized as $1.5 \mathrm{~mm} / 2 \mathrm{~mm}$ with Ron $=20 \mathrm{~W}$, and the pmos is sized as $3 \mathrm{~mm} / 2 \mathrm{~mm}$ with Ron $=25 \mathrm{~W}$. Special care was taken in the device layout to capture stray body diode currents and channel all currents directly to the power pads, as shown in Figure 3-10.

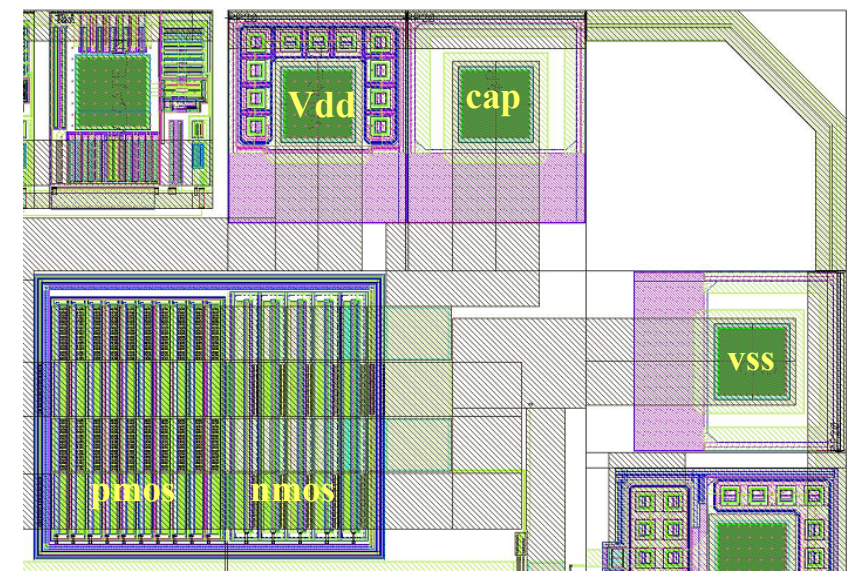

Figure 3-10. CAD layout of the primary $n \operatorname{mos}\left(M_{1} \& M_{3}\right)$ and pmos $\left(M_{2} \& M_{4}\right)$ switches

\section{Current-Mode Detector \& Comparator}

The current-mode detector illustrated in Figure 3-9 detects the signaling and handshaking currents during the rising and falling transitions. If adaptive deadtime control is desired, the current detector also needs to detect the transition current.

The primary challenges in detecting currents in the capacitor switches include high speed requirements (order of $10 \mathrm{~ns}$ delay), bi-directional current detection, and the ability to withstand and quickly recover from large negative currents caused by mid-point transitions. Consider for example M1 in Figure 3-9. As a LSIC, the handshaking current flows positive from drain to source and the large transition current flows negative from source (ground) to drain. As a HSIC, the signaling current flows negative from source to drain. Thus to use the same hardware for both ICs, the circuit must be capable of detecting both positive and negative currents. The current polarities are shown for all switches in Figure 3-11 (note that the HSIC detects signaling currents and the LSIC detects handshaking currents).

A partial schematic of our solution is shown in Figure 3-12 for detecting the signaling and handshaking currents flowing through the nmos switch (M1 in Figure 3-9 and Figure 3-12). The 
basic concept is that M1, M5 and M6 operate in the on-state (triode) when the detector is active and are modeled as resistors R1 and R2 as shown in Figure 3-12. We then create an output current proportional to the sensed input current by forcing the voltage $v_{B}$ across M6 to match the voltage $v_{A}$, which equals the voltage across the primary device M1 plus a dc offset to accommodate negative input currents. The dc offset is created by M5 and a bias current Ib. The output current of the detector is given by (for $i_{\text {in }}>I_{1}, I_{b}$ )

$$
i_{\text {det }} \approx \frac{1}{2}\left(\frac{R_{1}}{R_{2}} i_{\text {in }}+I_{b}\right)
$$

\section{Equation 3-1}

where $\mathrm{R} 1 / \mathrm{R} 2=1 / 300$ and $\mathrm{Ib}=100 \mathrm{~mA}$ in the IC design. The scale factor is achieved using matched devices with care taken in the layout for good matching and reduced noise.

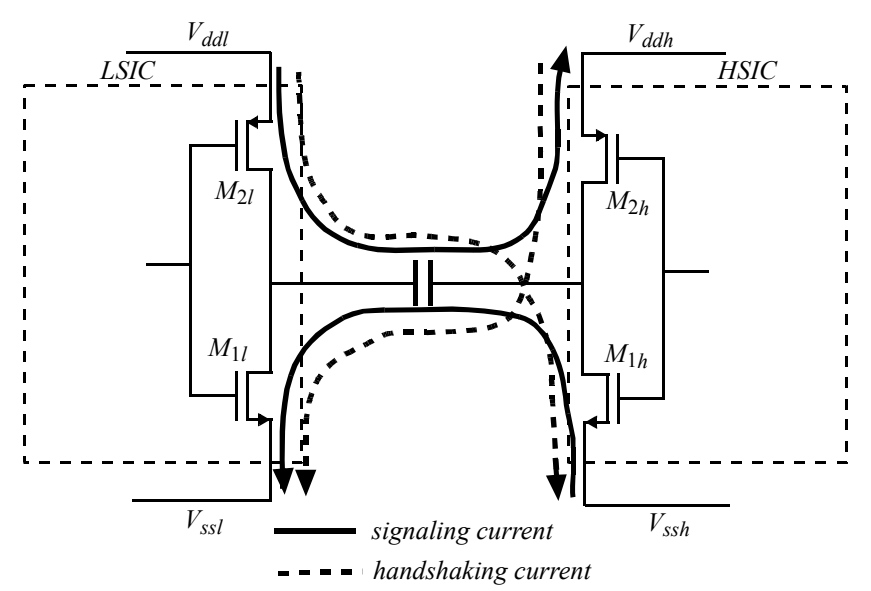

Figure 3-11. Signaling and handshaking current polarities in the LSIC and HSIC switches M1 \& M2

The detector current is then passed to a high-speed current-mode comparator, with the schematic shown in Figure 3-13. The reference current in the comparator sets the current detection thresholds and is pre-programmed based on LSIC or HSIC operation. The thresholds correspond to input currents of $+45 \mathrm{~mA}$ for LSIC mode and $-15 \mathrm{~mA}$ for HSIC mode. The lower current threshold was required in HSIC mode to guarantee detection before body diode conduction clamps the detection point. The output of the comparator vdet is input to the control logic to drive state transitions. The comparator operates on the internal Vdd5 supply from the voltage regulator to interface with the low voltage digital logic $(5 \mathrm{~V})$. The circuitry and operation of the pmos detector are a direct dual of the nmos detector described in Figure 3-12. 


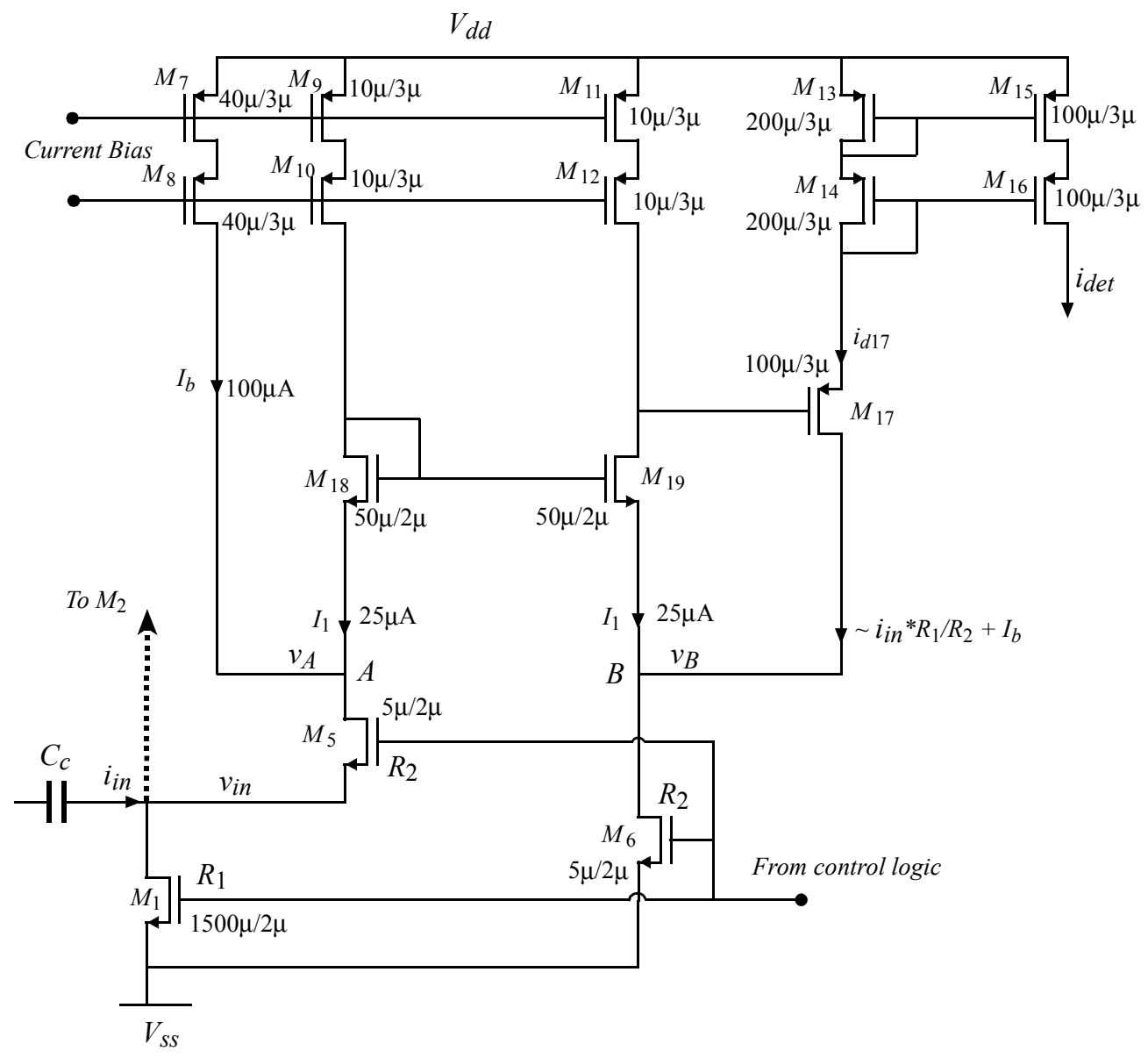

Figure 3-12. Schematic diagram of the bi-directional current detector for the nmos switch $M_{1} ; A$ direct dual circuit was used for sensing bi-directional current in the pmos device $\mathbf{M}_{2}$

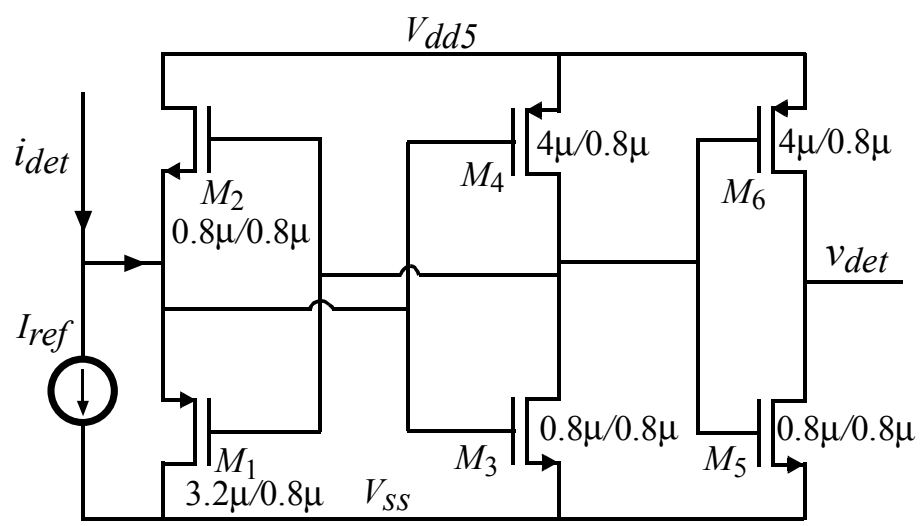

Figure 3-13. Schematic diagram of the fast current-mode comparator circuit. Feedback is used to maintain a low impedance input node for high-speed response with output buffers for sharp output edges. 


\section{Control Logic}

The control logic performs the state machine operation based on the control input and the outputs of the current detectors to generate the control signals for the switches. It also needs to handle fault conditions to avoid the simultaneous conduction of both power switches. The logic circuit is initiated by the reset command from the voltage regulator under voltage lock out (UVLO) circuit. Since the current polarities and detection pulses are different for the LSIC and HSIC designs as shown in Figure 3-11, separate state machines are used for each. The control logic are described in Verilog and then synthesized to the gate-level schematics through Cadence PKS (physically knowledgeable synthesis) and auto place and route.

The LSIC state machine is shown in Figure 3-14(a), where state transitions are edge triggered by the current comparator outputs vdet_n and vdet_p. We chose to use these asynchronous signals for state transitions due to the ns speed requirements associated with signaling detection and response. A traditional synchronous clock state machine design would require a very high frequency clock with significant power consumption and on-chip EMI. The state transitions operate as discussed in Section 3.1.1, with selection pins used for pre-programmed parameters initial_time (at startup), fault_time (time-out for expected transitions), mask_time (masking of known high noise periods during transitions to avoid false detection) and deadtime (for gate drive). For the LSIC, the cap output is a direct logic inverse of the gate drive control input. The HSIC state machine is shown in Figure 3-14(b) with similar transition and parameter control as the LSIC, although the cap output is driven by the state machine in the HSIC.

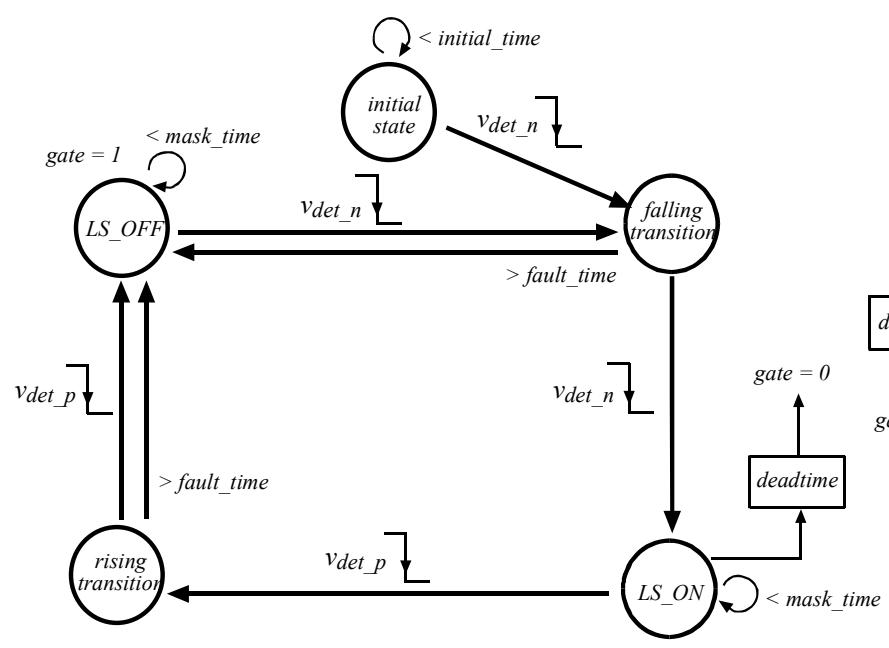

(a)

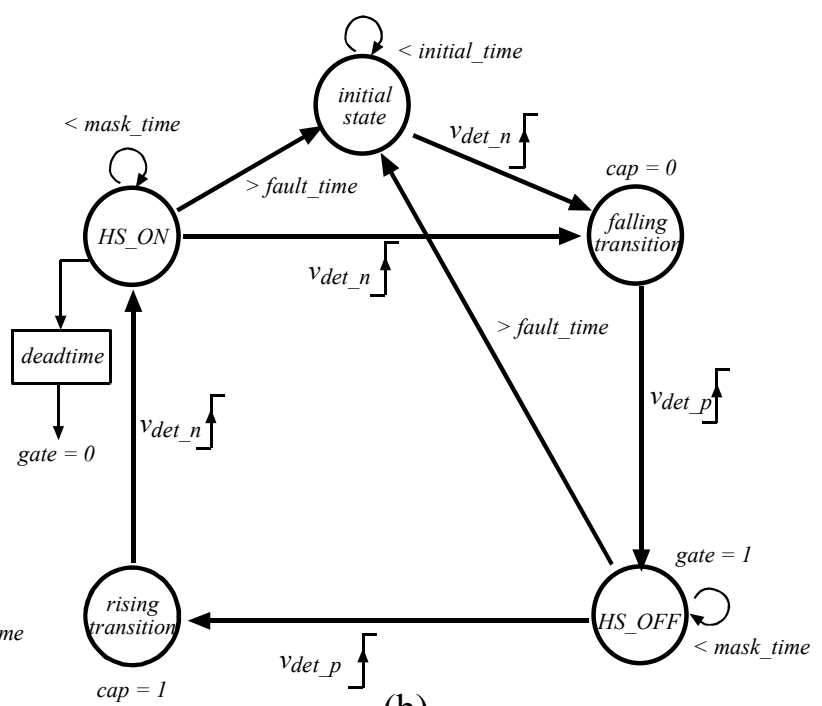

(b)

Figure 3-14. Sate transition diagram for a) LSIC, and b) HSIC. 


\section{Auxiliary Circuits}

Auxiliary circuits necessary for IC implementation are also included. A level-shifter is required to convert the outputs of the control logic from $5 \mathrm{~V}$ to $\mathrm{Vdd}(8 \mathrm{~V} \sim 15 \mathrm{~V})$ to drive the switches. A buffer made up of a chain of sized-up inverters is interfaced between the level-shifter and the gates of the switches to improve the driving capability. A linear voltage regulator is implemented on-chip to generate the $5 \mathrm{~V}$ supply for the digital logic and bandgap bias voltages and currents for the current detector. The voltage regulator also includes a power-on-reset module (UVLO), which creates a reset signal to initiate the state machine when the power supply is building up. A $20 \mathrm{MHz}$ on-chip clock is implemented to generate the selectable timing constants.

A microphotograph of the complete custom IC fabricated in the Austria-Microsystems (AMS) $0.8 \mathrm{~mm}$ CMOS process is shown in Figure 3-15 with each core functional block labeled.

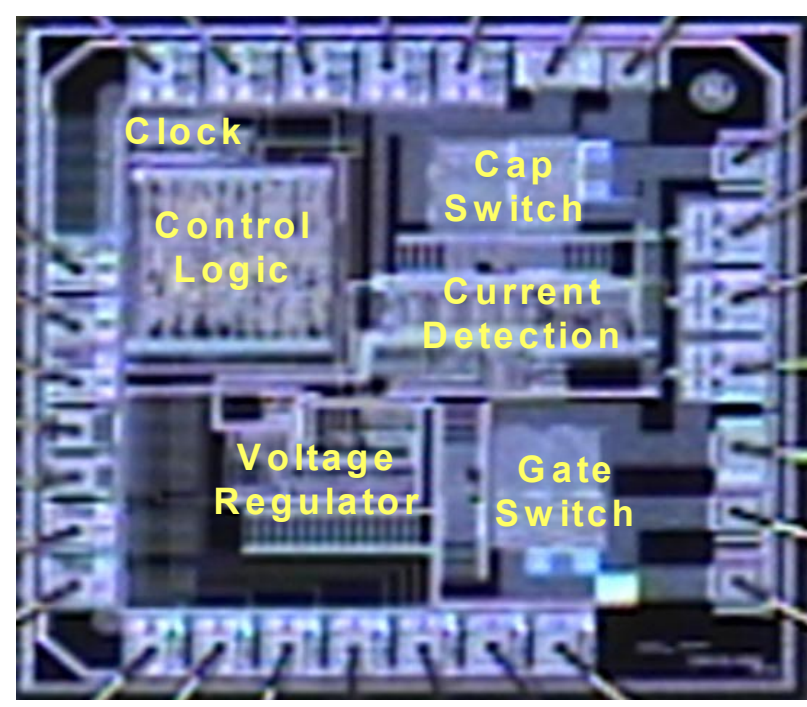

Figure 3-15. Microphotograph of custom IC fabricated in the AMS 0.8 $\mu \mathrm{m}$ CMOS process with core blocks labeled.

\subsubsection{Gate Drive Custom IC Simulation Results}

The simulation was performed in the Cadence analog simulation environment. The design was verified through the simulation of each functional block as well as the whole system. The process and temperature corner simulation examines all analog designs. Some key simulation results are highlighted as follows.

Figure 3-16 and Figure 3-17 illustrate the corner simulation results for the current detector with $\mathrm{R} 1 / \mathrm{R} 2=1 / 300$ and $\mathrm{Ib}=100 \mathrm{uA}$. The input current is emulated by a trapezoidal waveform. Both positive and negative inputs are simulated through 27-points process corner analysis. The signals shown are identical to those defined in Figure 3-12. The simulation shows that the delay of the 
detector is around 10ns. The output current for the positive input current is scaled down approximately by a factor of 300 , which is in agreement with the analysis. The negative detection shows some distortion around the peak of the input current. This is because VB is clamped at zero and cannot swing to a negative value as the input current increases. However, as the current detector only performs threshold detection, the distortion around the peak of the input current does not affect the output of the current comparator as long as the input current below the threshold does not lead to clamp and distortion. Another potential problem for the negative detection is the conduction of the body diode, which happens when the input negative current is very large and results in clamping voltage VA. To get around this problem, the switch is sized large enough to avoid the conduction of the body diode for the input current below the current threshold.

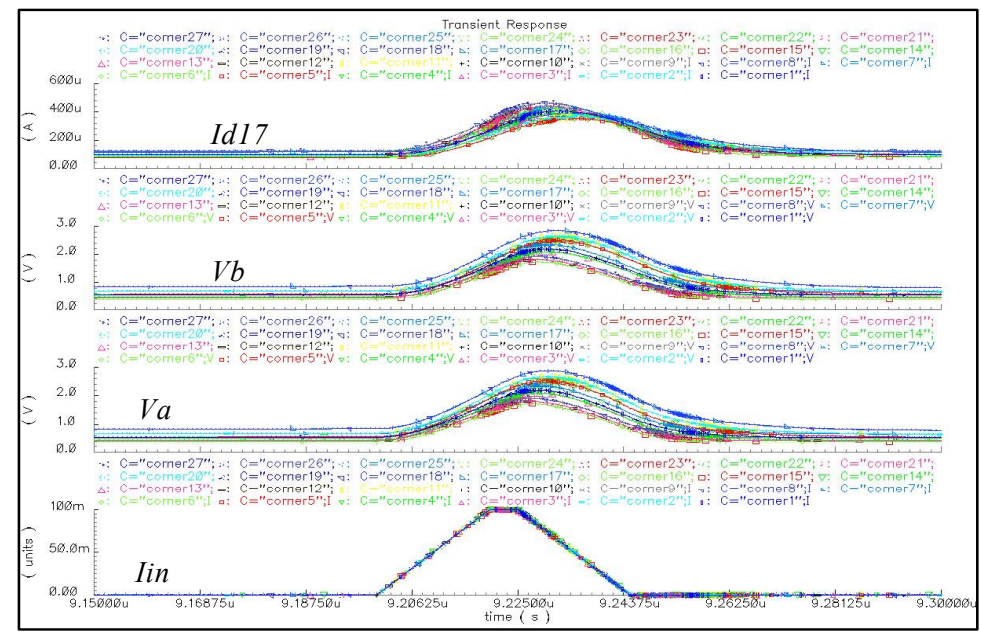

Figure 3-16. Corner simulation results of current detector for positive current

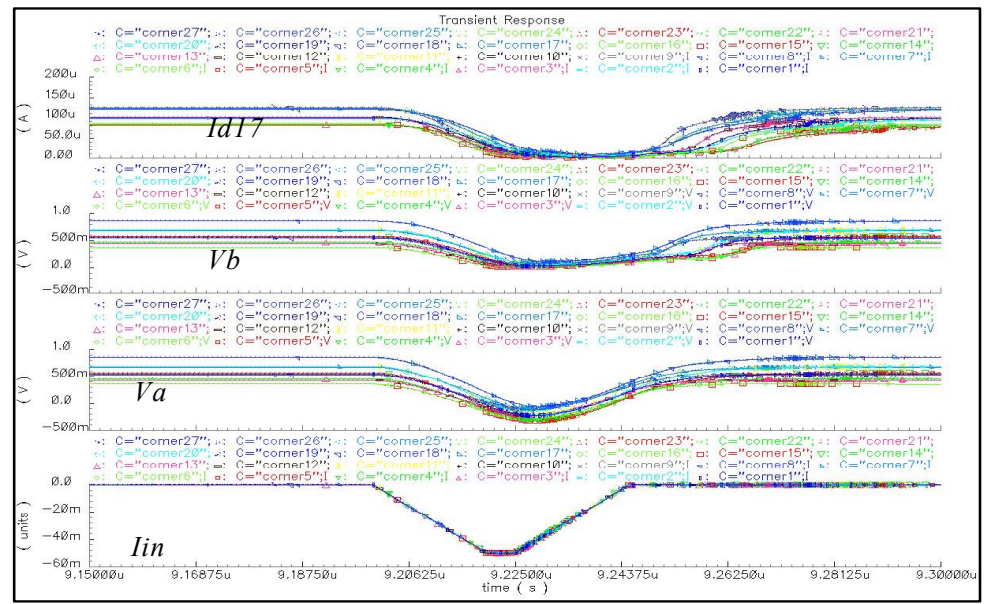

Figure 3-17. Corner simulation results of current detector for negative input current 
Figure 3-18 and Figure 3-19 present the simulation results for the digital control logic. The inputs to the digital controller are emulated by independent pulse sources, which are similar to the signals generated by current detector and comparator. It can be seen that the digital controller creates proper control signals to drive the capacitor (cap) and power switches (gate) by sequencing through four operation states.

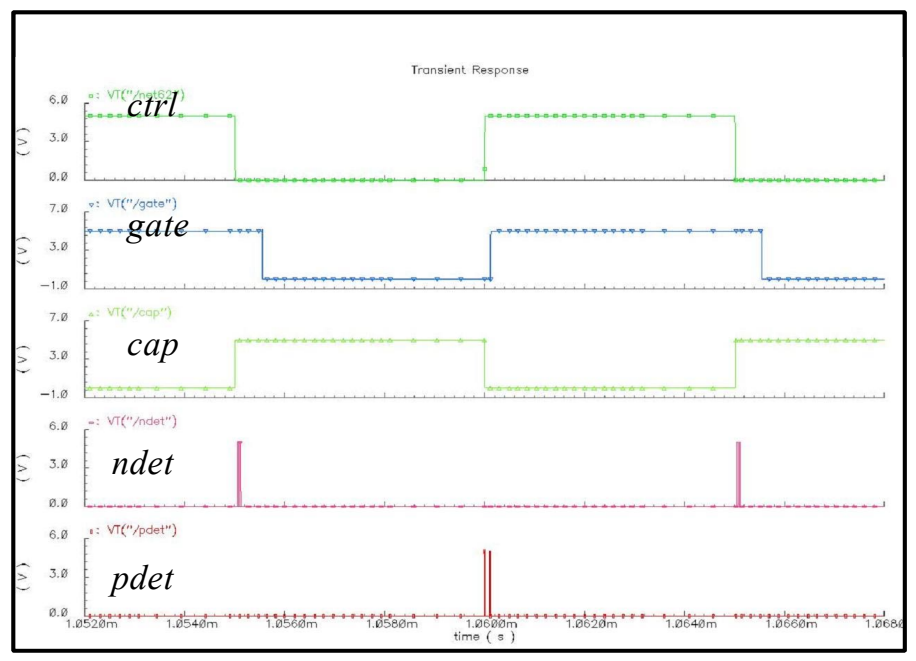

Figure 3-18. Simulation results of low-side digital controller

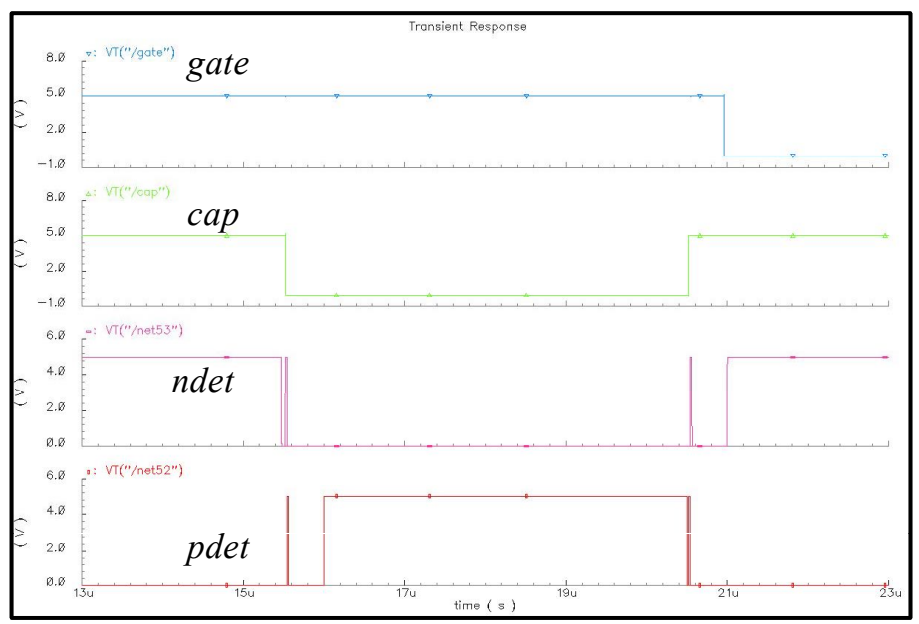

Figure 3-19. Simulation results of high-side digital controller

The system simulation is performed with the gate driver and half-bridge LCC ballast, as shown in Figure 3-20 and Figure 3-19. The low side and high side ICs are coupled through a 400pF capacitor. Since the design for digital control logic is synthesized to standard cells that contain global Vdd and ground, the high side logic cannot be floated. To solve this problem, the high side digital logic is grounded and coupled to the high side IC through an interface block, which is a VerilogA (analog Verilog) module and similar to an opto-coupler in functionalities. The ICs 
are supplied by ideal voltage sources. Figure 3-21 shows the simulation results for gate driving and inverter waveforms at $120 \mathrm{kHz}$. It can be seen that the gate driver follows the control input to switch the half-bridge network accordingly. The coupling capacitor current and the signals generated by the comparator are shown in Figure 3-22.

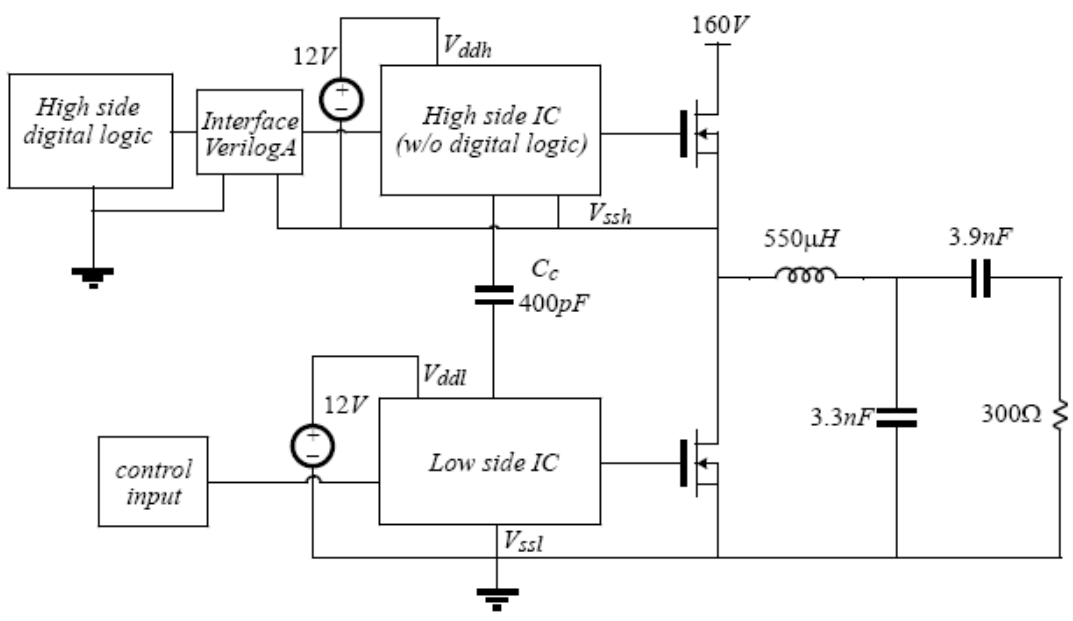

Figure 3-20. Simulation setup for driving half-bridge electronic ballast

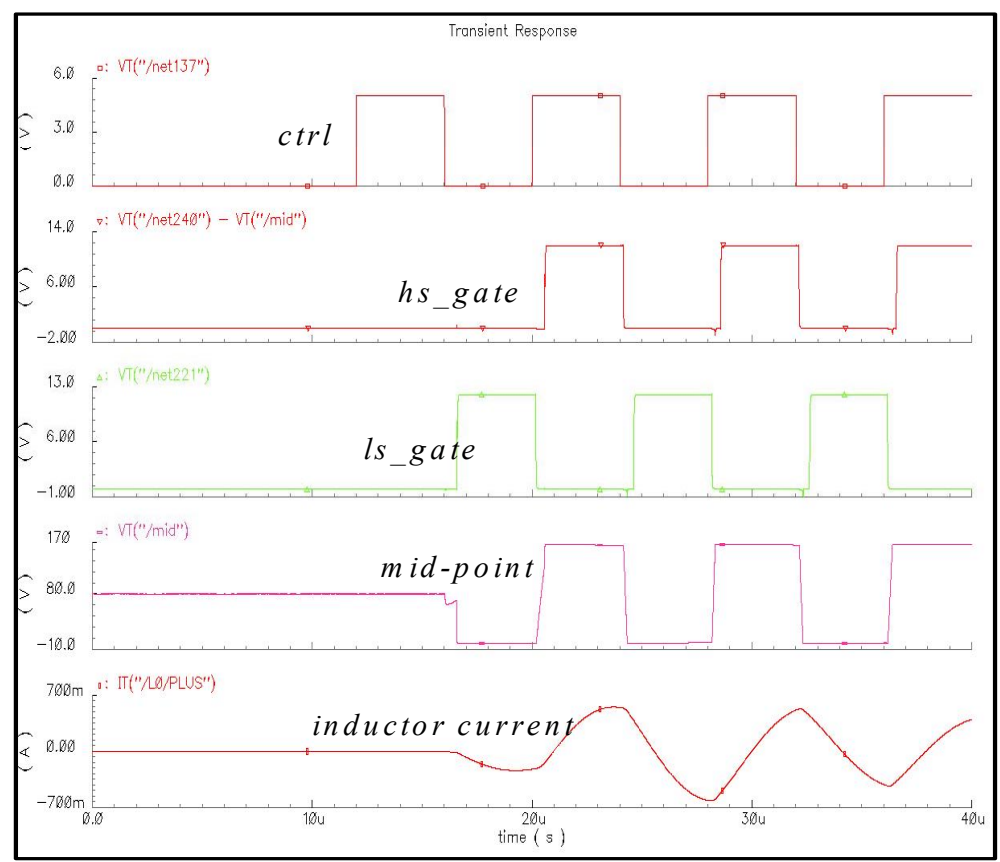

Figure 3-21. System simulation results of LCC ballast: gate drive and resonant waveforms 


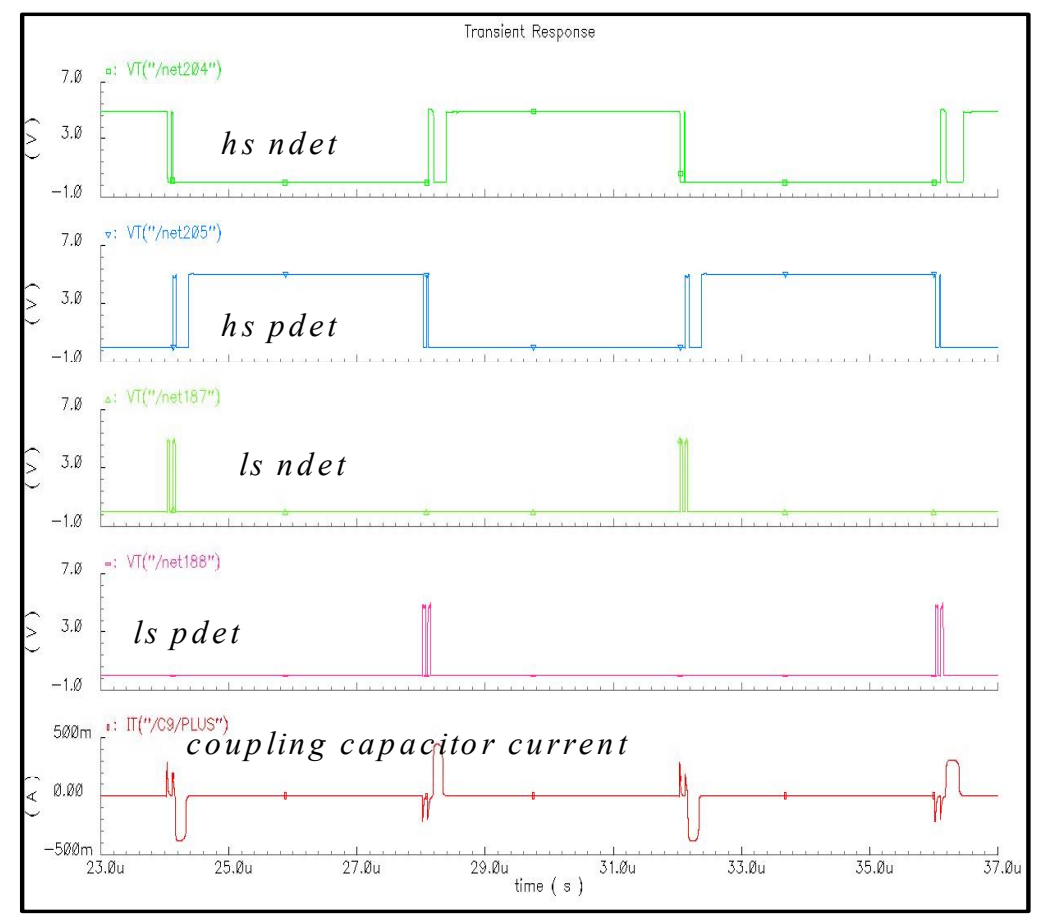

Figure 3-22. System simulation of LCC ballast: capacitor current and comparator waveforms

\subsection{Ballast Controller with Digital Phase Control}

Common approaches for controlling the electronic ballasts include control of duty cycle, phase angle between the midpoint voltage and the resonant inductor voltage/current, and switching frequency, where frequency control is one of the most popular control schemes. By changing the switching frequency, one can control the magnitude and phase of the output current and voltage. However, direct frequency control results in a number of disadvantages, including high sensitivity near resonance, strong dependence on resonant tank component values, and the requirement for additional control circuitry to detect the operation below resonance to prevent non-ZVS conditions. Phase control scheme on the other hand controls the switching frequency indirectly through the regulation of the inductor current or voltage phase angle relative to the midpoint voltage. This is the basic principle for the operation of self-oscillating ballasts, which provides the advantages of self-tuning to the resonant frequency and inherent ZVS operation. The major disadvantages of conventional self-oscillating ballasts include large number of discrete parts, sensitive to component variation, lack of control functionality and long product development cycle.

Some analog IC controllers are available in the market that greatly reduces the size and cost. Recently, interests have been building to provide direct digital control for power converters to achieve the benefits of reduced dependence on process and temperature, rapid design cycles, and real-time re-programmability in the controller operation. The digital implementation of the ballast controller can utilize the advantages of digital technology to further reduce the costs, 
incorporate more advanced and intelligent features as lamp protection, EMI reduction, and remote control and monitoring.

\subsubsection{Inductor Phase Control}

Figure 3-23 shows a simplified electronic ballast configuration consisting of a dc voltage source, switch network, resonant tank and the discharge lamp load. The dc voltage usually comes from ac line through an ac-dc rectifier. The switch network converts the dc voltage into a series of HF square-wave voltage (midpoint voltage for half-bridge ballast) by the gate driver circuit discussed in 3.1. The HF square-wave voltage is then fed into the resonant tank, thereby producing a high resonant voltage for the lamp ignition and matching impedance for the stable lamp operation. When the inverter operates above the resonant frequency $f_{0}$, the series inductor dominates the resonant tank such that the input impedance of the inverter is inductive as shown in Figure 3-24. Hence, the inductor current $i_{L}$ lags (and the inductor voltage $v_{L}$ leads) the midpoint voltage $v_{\mathrm{s}}$ from $0^{\circ}$ to $90^{\circ}$ as the switching frequency shifts away from the resonance. The output power decreases when the phase angle increases from $0^{\circ}$ to $90^{\circ}$. Thus it is possible to control the output power through the direct control of the phase angle between the midpoint voltage and the inductor voltage or current. The switching frequency is then indirectly controlled through the phase control.

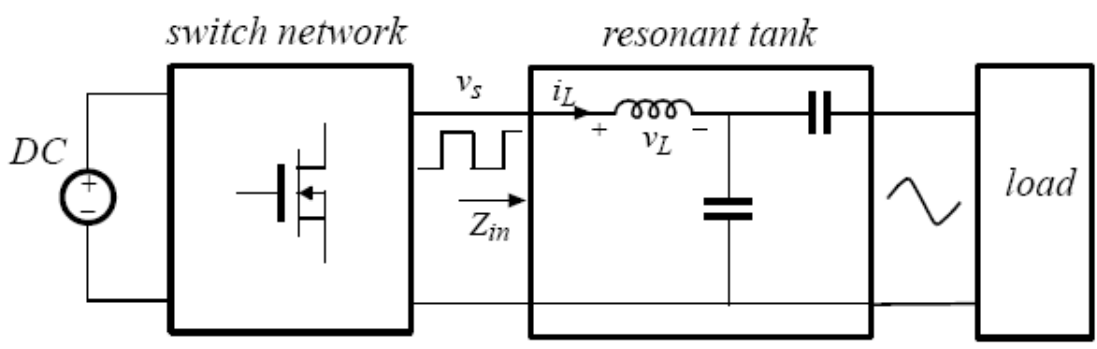

Figure 3-23. Simplified HF electronic ballast configuration with LCC resonant tank

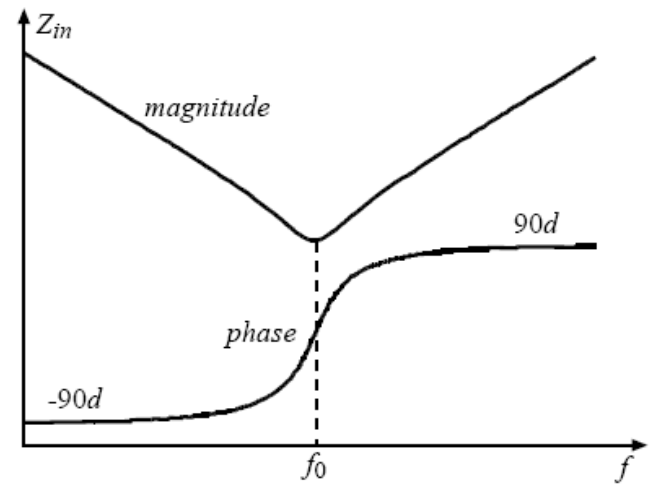

Figure 3-24. Bode plot of input impedance of a typical resonant tank 
By controlling the phase angle between the midpoint voltage and the inductor current to be greater than $0^{\circ}$, the system is forced to operate above the resonant frequency, resulting in ZVS operation for appropriately designed resonant tanks. If the resonant frequency shifts due to variations in the tank elements and the load, the phase controller is able to self-tune to the resonant frequency and the operating point is not affected significantly. The phase control also provides a possibility to apply the same controller to resonant inverters with different topologies and resonant frequencies. The self-tuning feature of the phase control can automatically adjust the switching frequency to adapt the change of the resonant frequency on a near cycle-by-cycle basis and the operating point will not be affected, which cannot be achieved through the direct frequency control.

Phase control can be performed through sensing either the inductor current or voltage. Current sensing gives more accurate results since the inductor current is nearly sinusoidal, but it requires a current transformer (CT), which is not desired for some applications. Inductor voltage sensing is applied with the assumption that the inductor voltage leads the inductor current by $90^{\circ}$ (it is true for a sinusoidal system, but approximate for a near-sinusoidal system with higher-order harmonics like the resonant inverter). Voltage sensing results in more significant phase error due to the larger harmonics in the inductor voltage, as the inductor is usually connected to the switching network. The primary motivation for inductor voltage sensing is the ability to use an additional winding on the resonant inductor for low cost and lossless sensing. Inductor current phase scheme will be discussed in detail in the following section.

\subsubsection{Direct Digital Phase Control Scheme}

Figure 3-25 shows a block diagram of direct digital phase control scheme with inductor current sensing. The phase controller receives digital phase reference, $\varphi_{c}$, and regulates the phase angle on a near cycle-by-cycle basis to the desired value. The waveforms associated are shown in Figure 3-26, from which basic strategy to directly control the inductor current can be derived.

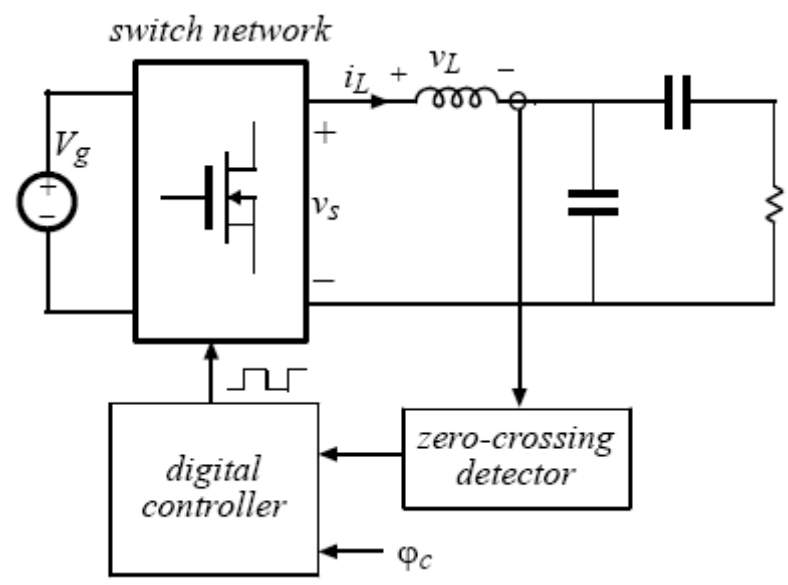

Figure 3-25. Direct digital phase control scheme with inductor current sensing 
The essence of the control is to time the period by detecting the inductor current zero crossing, then compute the required time delays from the zero crossing to determine when to turn on or off the high and low side gate drives to achieve the desired phase. Given a phase command, $\varphi_{c}$, the time delay from the zero crossing of the inductor current to the falling edge of the midpoint voltage can be computed as

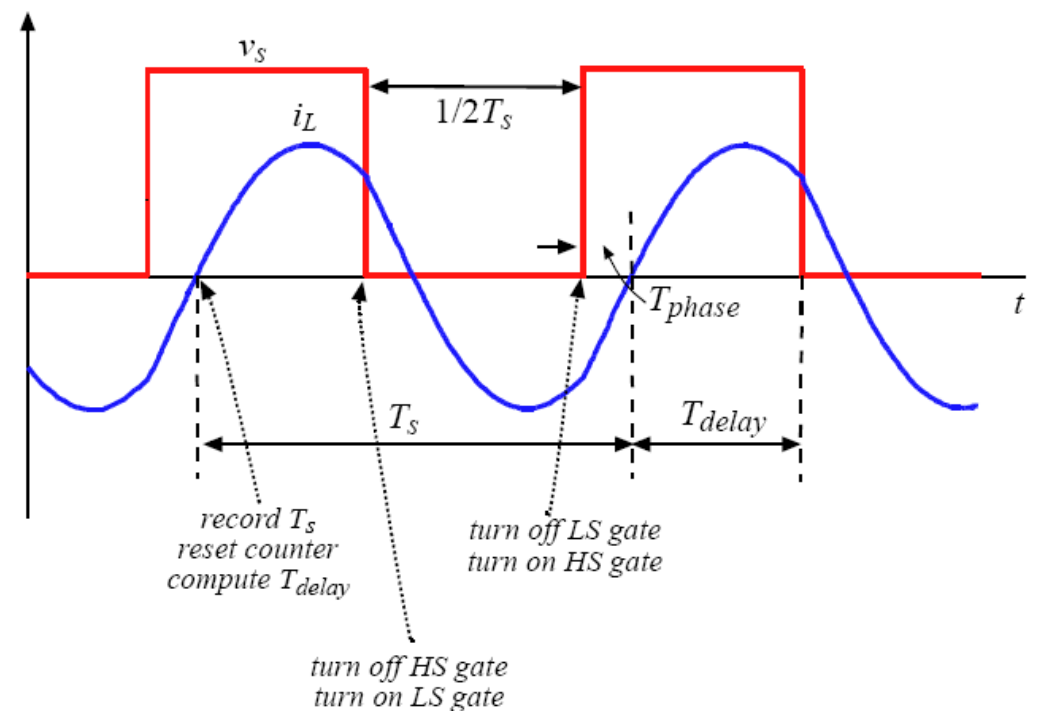

Figure 3-26. Phase control in a half-bridge LCC ballast using inductor current and midpoint voltage.

$$
T_{\text {delay }}=\frac{T_{s}}{2}-\varphi_{c} \times \frac{T_{s}}{360^{0}}=\frac{T_{s}}{4}(2-\alpha)
$$

\section{Equation 3-2}

where $\alpha=\varphi_{\mathrm{c}} / 90^{\circ}$.

Given the period $T_{s}$ and the phase command, $\varphi_{c}$, Equation 3-2 can be used to compute $T_{\text {delay }}$. The controller can obtain the period $T_{s}$ by recording the time between two adjacent positive (or negative) zero-crossings of the inductor current as shown in Figure 3-26. Hence, a control scheme based on the inductor current sensing is proposed as:

a. Detect the zero crossing of the inductor current using a high-speed comparator;

b. Record $T_{s}$ of the previous cycle, which is the time interval between two zero-crossing points;

c. Compute $T_{\text {delay }}$ according to Equation 3-2; 
d. Wait for $T_{\text {delay }}$, then turn off the high side (HS) gate and turn on the low side (LS) gate with a proper deadtime;

e. Wait for half of Ts, then turn off the LS gate and turn on the HS gate with a proper deadtime;

f. Wait for next zero crossing of the inductor current, then repeat this cycle.

This control scheme is very easy to implement in a digital controller. For digital control, Equation 3-2 is revised in a discrete form as

$$
T_{\text {delay }}[n]=\frac{T_{s}[n-1]}{4}(2-\alpha[n])
$$

Equation 3-3

As $0 \leq \alpha[\mathrm{n}] \leq 1$, Equation 3-3 can be further revised to facilitate the digital implementation as

$$
T_{\text {delay }}[n]=\frac{T_{s}[n-1]}{4} \frac{\left(2^{m}-1\right)+\left(2^{m}-1-\alpha_{1}[n]\right)}{2^{m}}
$$

Equation 3-4

where $0 \leq \alpha_{1}[n] \leq 2^{m}-1, m$ is the number of bits for the digital phase command, and $\alpha_{1}[n]=0$ corresponds to $0^{\circ}$ while $\alpha_{1}[\mathrm{n}]=2^{\mathrm{m}}-1$ corresponds to $90^{\circ}$. A small error exists in Equation 3-4 due to a denominator of $2^{\mathrm{m}}$ instead of $2^{\mathrm{m}}$-1, which requires significantly larger silicon area to implement the divider instead of an m-bit shifter in hardware. For large $\mathrm{m}$, this error can be ignored. From Equation 3-4, it can be seen that the switching period of previous cycle is used to compute the delay of current cycle.

Equation 3-4 is basically the control law for the proposed digital phase control scheme. This control equation can be easily realized with standard digital logic and implemented in a custom CMOS digital IC or programmable logic. It can also be implemented in a microcontroller or DSP. In addition to the digital logic required to implement the control functions, the zerocrossing detector is also necessary. It can be as simple as an off-chip or on-chip comparator.

One practical improvement that is easily implemented in a digital system is to actively cancel the signal propagation delay of the gate driver $(\sim 100 \mathrm{~ns})$. This is achieved by measuring the delay between the gate drive command sent out by the controller and the actual gate drive signal appeared at the power switch gate for each switching cycle, and modifying Equation 3-4 as

$$
T_{\text {delay }}[n]=\frac{T_{s}[n-1]}{4} \frac{\left(2^{m}-1\right)+\left(2^{m}-1-\alpha_{1}[n]\right)}{2^{m}}-T_{g d}[n-1]
$$

\section{Equation 3-5}


where $T_{g d}[n-1]$ is the gate driver delay of previous cycle obtained by feeding the LS gate drive signal back to the digital controller.

\subsubsection{FPGA-Based Verification of Digital Phase Control}

The digital phase control scheme is verified through the development board for Xilinx FPGA Virtex II xc2v1000 to provide rapid prototyping with realistic hardware realization.

The digital control algorithm with inductor current sensing can be represented by a synchronous state machine as shown in Figure 3-27. A counter is used to record the period and control the timing. The minimum time step is determined by the system clock frequency. A $100 \mathrm{MHz}$ clock is used with $10 \mathrm{~ns}$ control resolution. The state machine outputs two complementary gate driver signals with preset dead time to control the gate driver.

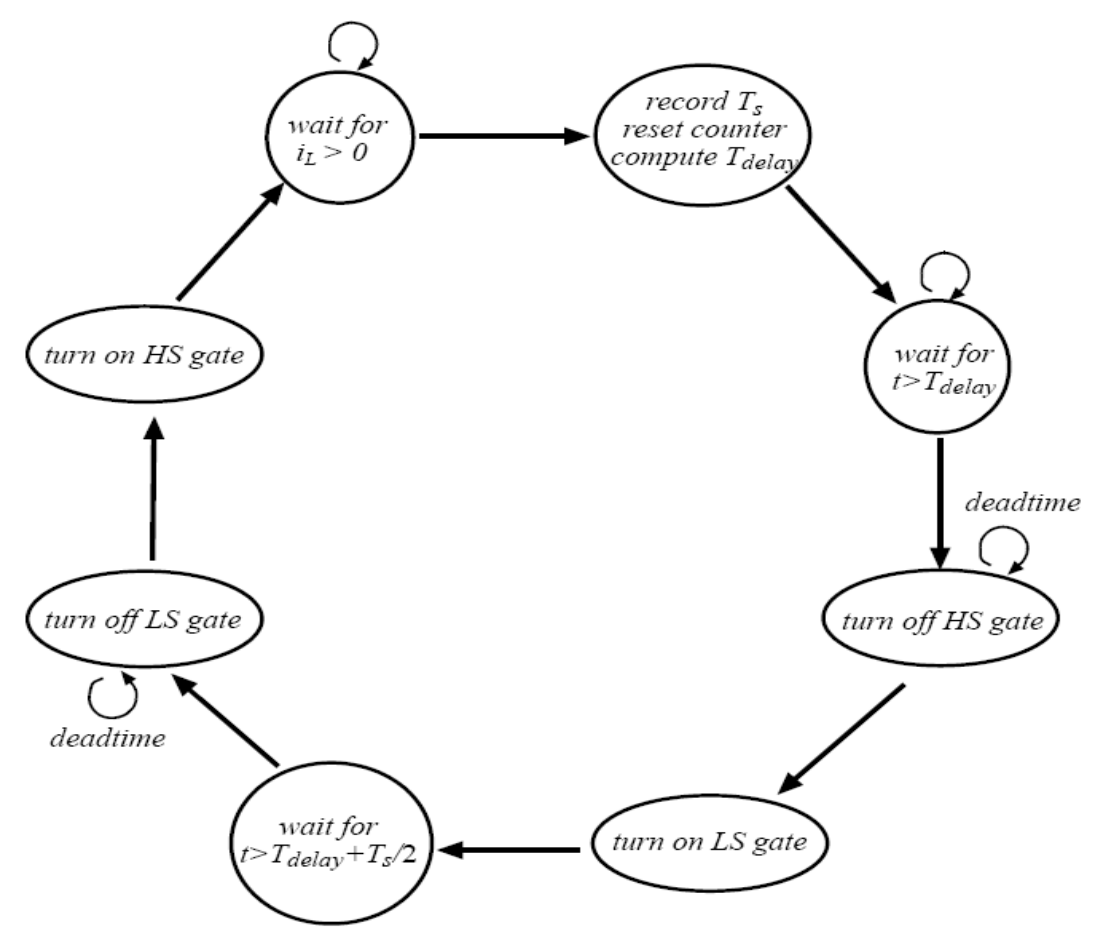

Figure 3-27. State machine of phase controller with inductor current sensing

The complete digital controller is described with the Verilog hardware description language (HDL), making synthesis and hardware realization (placing and routing) fully automated. The zero-crossing detection of the inductor current is performed through a current transformer and an off-chip comparator. The FPGA-based controller controls a half-bridge gate driver to drive the power switches. 
The complete experimental testing system is shown in Figure 3-28, including LCC half-bridge ballast with $40 \Omega$ resistive load, current transformer, zero-crossing detector (comparator), FPGAbased digital controller, and gate driver. Phase command $\alpha_{1}[\mathrm{n}]$ is selected to be 8 bits $(m=8)$. Figure 3-29 illustrates the steady-state operation waveforms for three different phase commands with active gate driver delay cancellation. It can be seen that the control of the phase does result in the control of the switching frequency and the magnitude of the current. The difference between the phase command and the measured phase is less than $2^{\circ}$, which is mainly introduced by the comparator delay and internal control delay. The performance of step responses is shown in Figure 3-30.

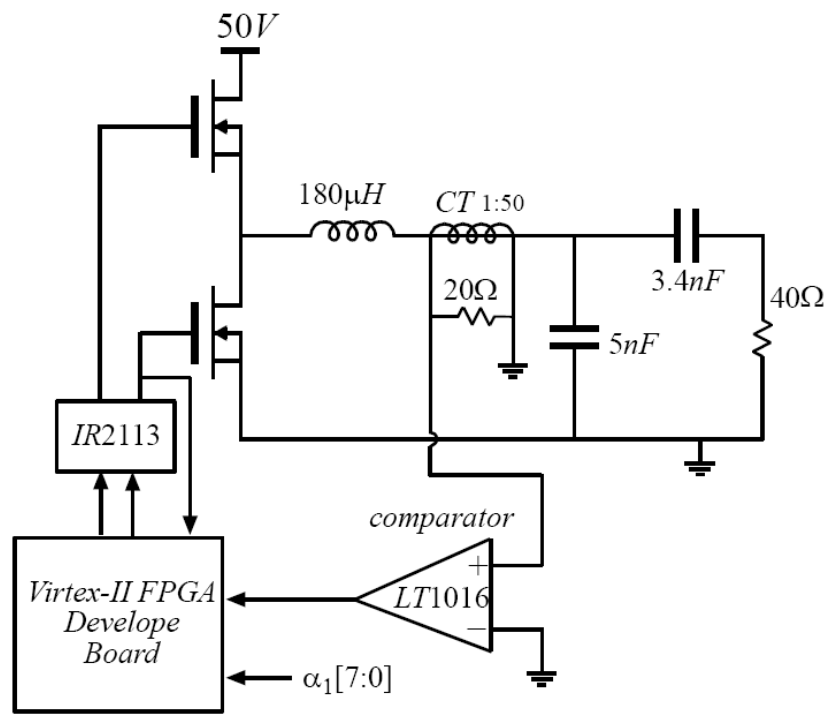

Figure 3-28. FPGA-based verification circuit for direct digital phase control 


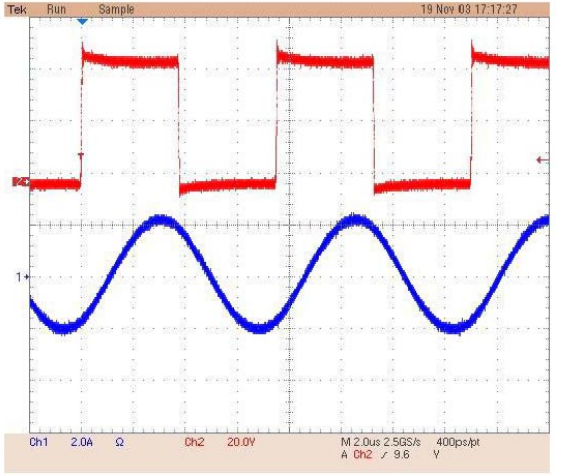

(a)

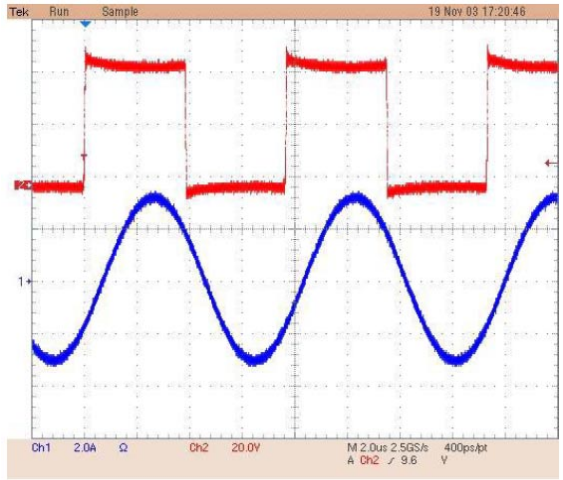

(b)

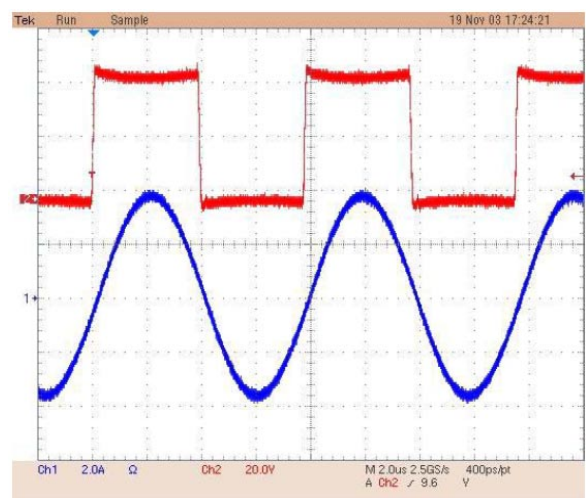

(c)

Figure 3-29. Steady-state waveforms of midpoint voltage and inductor current for different phase command, ch1: inductor current, $2 \mathrm{~A} / \mathrm{div}$; ch2: midpoint voltage, $20 \mathrm{~V}$.div; a): $56.25^{\circ}$ commanded, $5_{4.98^{\circ}}$ measured; b) $33.75^{\circ}$ commanded, $32.18^{\circ}$ measured; c) $11.25^{\circ}$ commanded, $10.12^{\circ}$ measured.

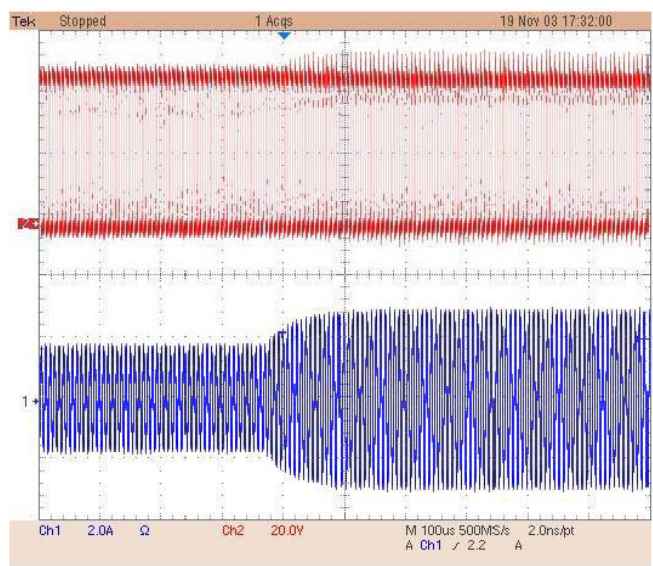

(a)

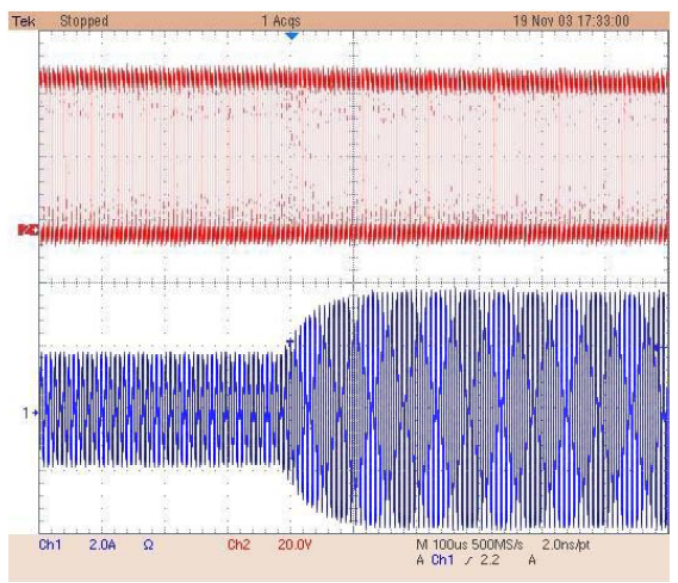

(b)

Figure 3-30. Set responses, a) $67.5^{\circ} \rightarrow 45^{\circ}$, b) $67.5^{\circ} \rightarrow 22.5^{\circ}$ 


\subsubsection{Ballast Controller Custom IC Design}

The ballast controller proposed is a two-loop system with an inner phase loop regulating the inductor current phase relative to the midpoint voltage and a outer loop controlling the lamp current or voltage by generating the phase command to the inner phase loop. Compared with the inner loop, the outer loop regulates the current envelope through a traditional compensator, and is thus slower than the inner phase loop.

Figure 3-31 shows the block diagram for the two-loop digital LCC ballast control system that can be generally applied to other types of resonant ballasts. The outer loop controller senses the output current (can also be voltage) and converts it to a digital signal through the A/D converter. The envelope detector then finds the envelope signal through the digital rectification and filtering. Compared with the analog counterpart, the digital envelope detector is more accurate and more efficient as it removes the effects of the diodes.

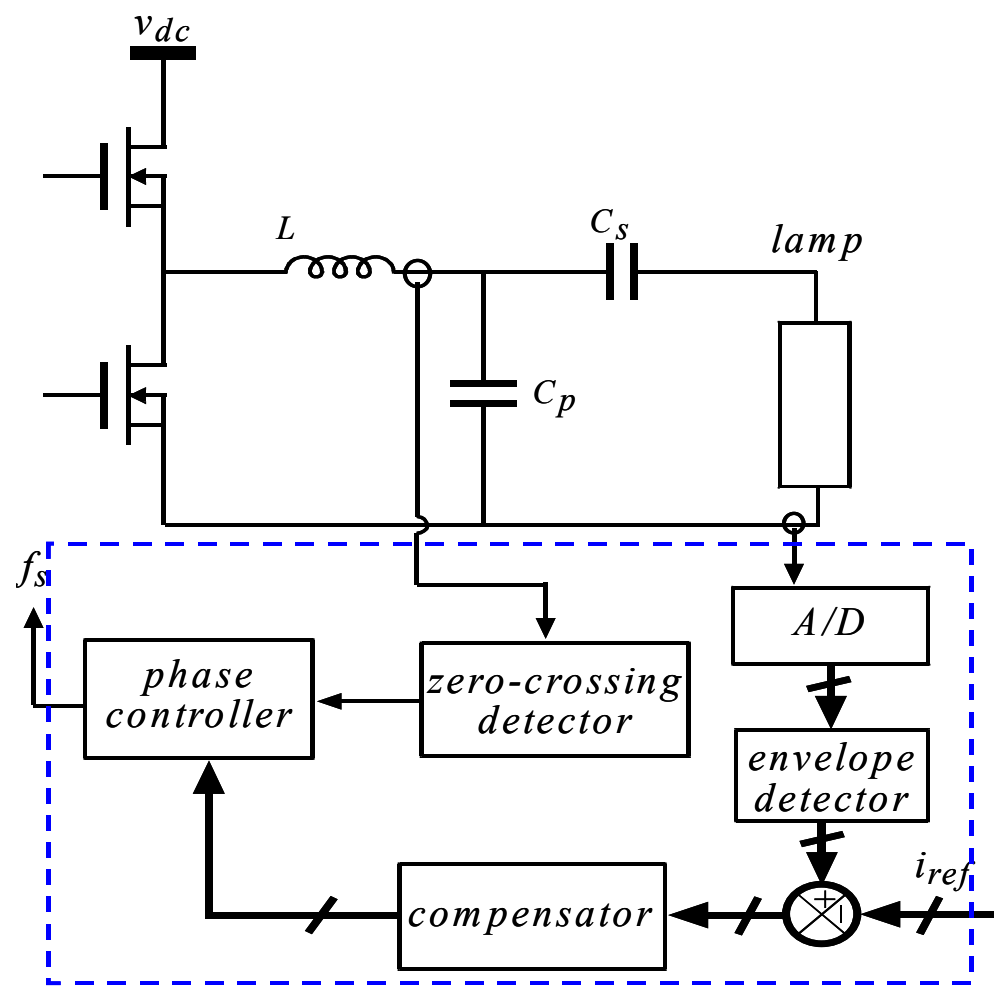

Figure 3-31. Simplified block diagram of the ballast controller IC with two primary loops: 1) inner phase control and 2) outer lamp current regulation.

The compensator design is heavily dependent on the dynamics of the ballast. Generally a PID type digital compensator is required to maintain the system stability and achieve fast response. As multiple bits are usually involved in the computation to obtain fine control resolution, a large multiplier or look-up table is generally required unless special design is implemented to reduce the size of the compensator. An effective approach is to limit the range of the error signal. This 
approach is based on the assumption that the system is normally operating under steady state and the error signal is very small. The compensator generates the phase reference for the inner phase loop. To extend the dynamic range, the internal computation can allow the phase to be out of $0^{\circ}$ $\sim 90^{\circ}$. Some preventive measures must be taken to avoid the output phase greater than $90^{\circ}$, which makes no sense, or lower than $0^{\circ}$, which means undesired below-resonance operation.

Due to the special characteristics of the discharge lamp, some auxiliary functions such as ignition, over voltage protection, and filament preheating for some types of lamps are required. The ignition (or start-up) controller generates a high voltage across the lamp to strike the plasma. To extend the lamp life, the soft starter that increases the lamp voltage gradually is desired. If the start-up process succeeds, the system enters the normal closed-loop operation and the lamp voltage drops down. If the start-up fails, the controller can either try to ignite the lamp again or shut down the system for safety. During the start-up or the normal operation, an excessive voltage may be applied to the system due to ignition failure or other exceptions (for instance, lamp removal or failure), resulting in the damage of the whole system. To avoid this situation, an over voltage protection function is introduced to monitor the lamp voltage. Once the lamp over voltage happens, the system will be immediately shut down. The controller will try to ignite the lamp after the over voltage is removed. For the HID ballast, the restart is not allowed until the lamp cools down. For some applications, a preheating stage may be required to warm up the filaments before the start-up controller tries to ignite the lamp. A small voltage is usually applied across the filament to supply some amount of current, which can heat the filament and help it emit hot electrons.

This two-loop ballast controller can also be extended to a three-loop configuration by incorporating the lamp power loop, which is required for some applications such as HPS (high pressure sodium) lamp. The lamp power controller regulates the lamp power through sensing the lamp current as well as the lamp voltage and generates the lamp current reference to control the lamp current feedback loop. The lamp current loop and the phase loop are identical to the twoloop controller.

The control functions can be implemented in a mixed-signal manner. The digital control functions are described with hardware description language (HDL) like Verilog. The digital controller is interfaced with the ballast through analog ac buffer, comparator, and A/D converter. A simplified block diagram of the core blocks is shown in Figure 3-32. The inner dashed box contains the core digital controller while the outer dashed box contains the analog interface functions. 


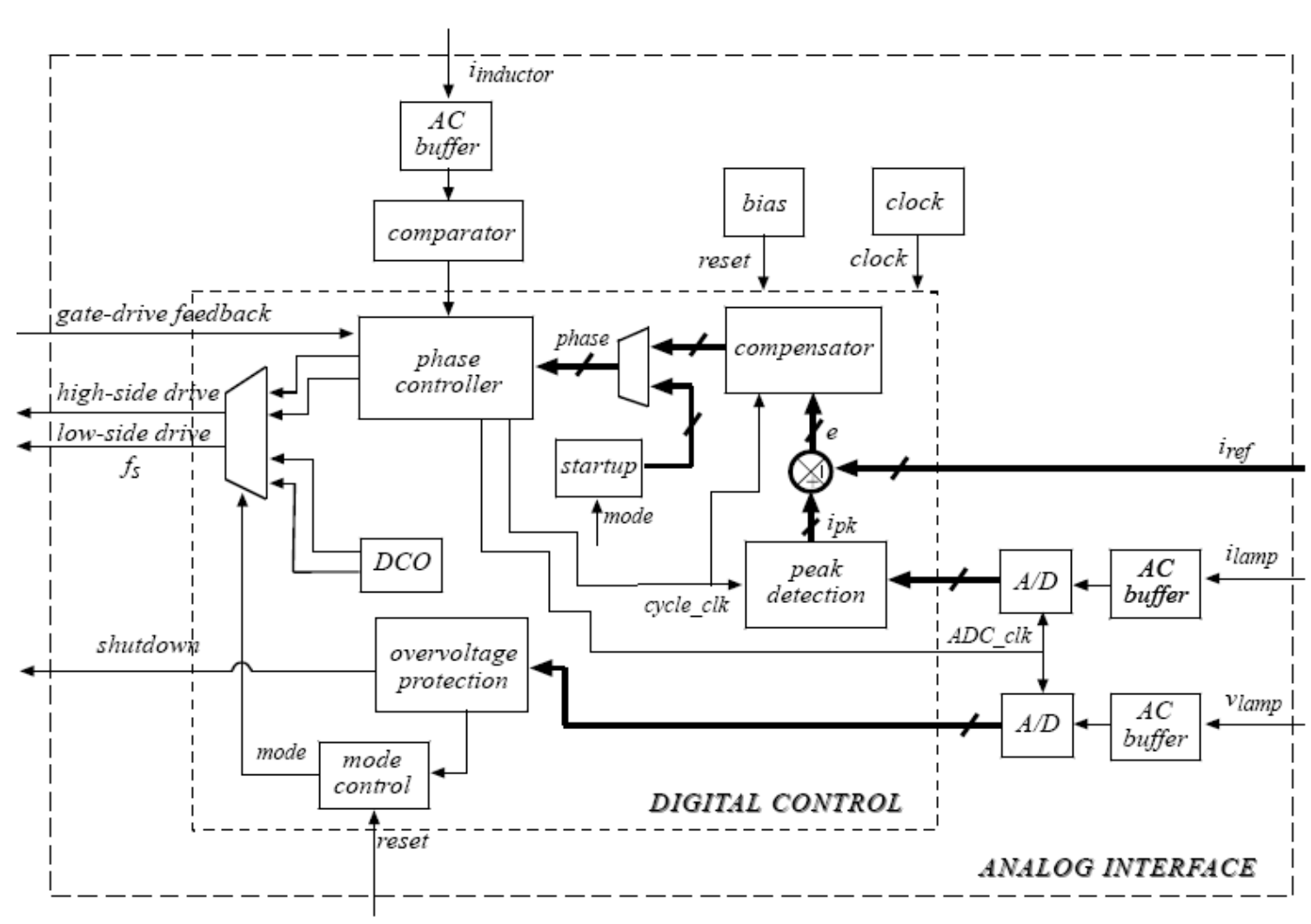

Figure 3-32. Block diagram of the ballast controller custom IC

\section{Digital Control Blocks}

The core digital controller of the IC receives zero crossing information on the inductor current, samples of the ac lamp current and voltage, low side gate drive feedback, and a digital reference current command, and outputs the high and low side gate drive signals and a shutdown signal for protection. The ballast control can be considered to operate in two modes: Lamp Ignition and Lamp Current Regulation. During lamp ignition, the digitally controlled oscillator (DCO) module guarantees initial operation to enable phase detection and regulation. The startup module then sweeps the phase from a high to a low value until the lamp ignites. Once the lamp ignites, the peak detector and compensator modules implement the digital current regulation loop. The over-voltage protection module monitors lamp voltage and shuts down the lamp should the voltage exceed a programmable limit. Although this module is active at all times, it is particularly relevant during lamp ignition and in the event of a lamp failure. The phase controller is active during both lamp ignition and lamp current regulation, regulating the phase angle between inductor current and midpoint voltage and providing supervisory timing functions for synchronization of analog-to-digital (A/D) conversion sampling, lamp current peak detection, and lamp current compensation. The phase command for the phase controller is provided by the startup module during lamp ignition (phase sweep) and by the compensator during lamp current regulation. Each of the core blocks active during lamp ignition and current regulation are described in more detail below. 


\section{A.1: Digital Phase Controller}

This inner loop implements the control algorithm shown in Figure 3-27. It provides a once percycle pulse based on inductor current positive zero crossing detection to synchronize the peak detector and compensator and a higher frequency synchronized clock for the A/D converter. The synchronous state machine is running at $100 \mathrm{MHz}$ generated by the on-chip clock generator. The phase command is 8 -bit provided by the closed-loop compensator.

\section{A.2: Lamp Ignition}

The DCO, startup, and over-voltage protection modules are active during lamp ignition. When the controller is first powered up, it will operate in DCO at a programmable frequency for $10 \mathrm{~ms}$. This is followed by the phase sweep. Once the peak lamp current exceeds a specified threshold $\left(i_{p k}>i_{t h}\right)$, the lamp current compensator takes over. The start phase, minimum phase, sweep rate, and threshold current are all programmable. The minimum phase setting is used to protect the ballast (in addition to over-voltage protection) and avoid zero voltage switching. Figure 3-33 shows a block diagram of the startup module, which performs the phase sweep. The start phase and minimum phase parameters, which define start and end values for the phase sweep, are not shown in the figure. The startup controller also includes the multiplexer, which determines whether the phase command is to be provided from the phase sweep or the compensator. Should the startup module reach minimum phase without lamp current exceeding the threshold, the ballast controller will drop back into DCO and repeat the ignition sequence. In the case of an over-voltage, the controller will likewise drop back into DCO and repeat the ignition sequence, as well as send a shutdown signal to the gate driver. Thus high voltage ignitions are avoided the sweep is repeated multiple times until the lamp ignites at a sufficiently low voltage.

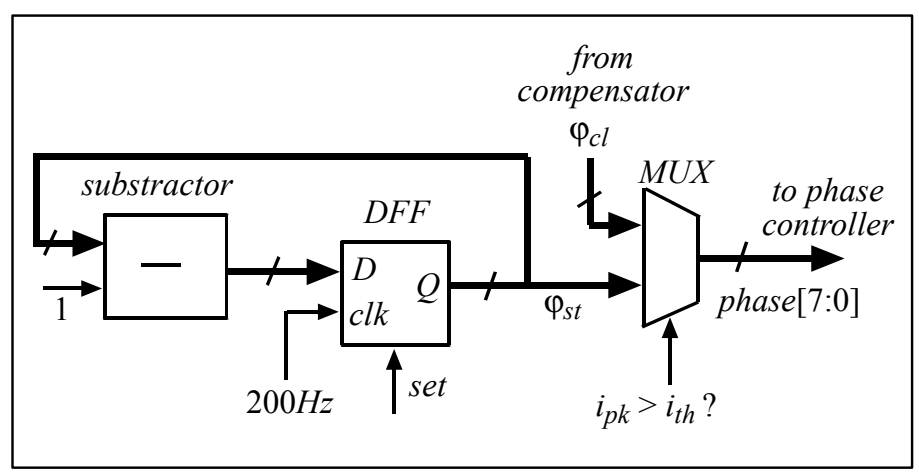

Figure 3-33. Block diagram of the startup controller, which sweeps phase from $90^{\circ}$ toward $0^{\circ}$

\section{A.3: $\quad$ Lamp Current Regulation}

Figure 3-34 and Figure 3-35 show block diagrams of the peak detector and compensator, respectively. The compensator is a simple integral only compensator with programmable gain. 
Both the peak detector output and compensator delay are latched by the once-per-cycle pulse, cycle_clk, from the phase controller. Although not shown in Figure 3-35, the programmable minimum phase parameter is used by the compensator as well as the startup module. Should the phase error be sufficient to cause the output of the compensator to reach minimum phase, the phase command will remain at minimum phase until the phase error decreases again. One problem with implementing the compensator of Figure 3-35 separately from the startup controller (including multiplexer) of Figure 3-33 is the potential for large steps in phase command when switching from the start-up phase sweep to current regulation. To address this problem, the start-up controller and compensator were combined into one module shown in Figure 3-36.

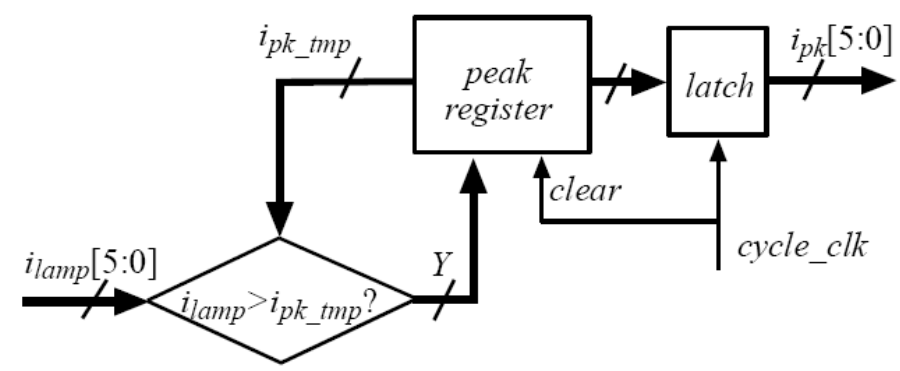

Figure 3-34. Block diagram of the peak detector, which latches the peak lamp current in each switching cycle

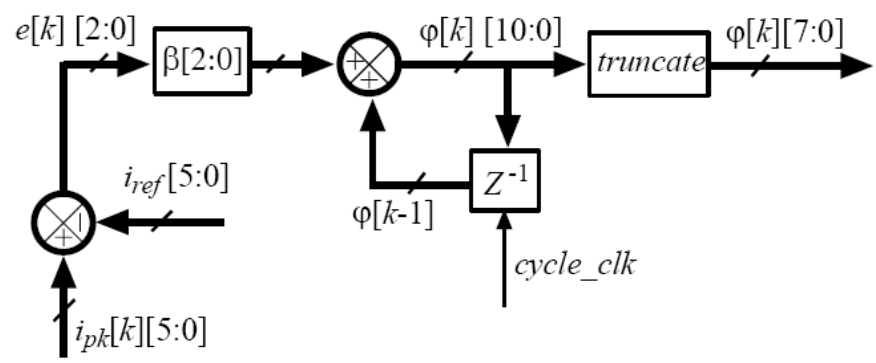

Figure 3-35. Block diagram of the compensator block, which provides integral compensation to the lamp current regulation loop 


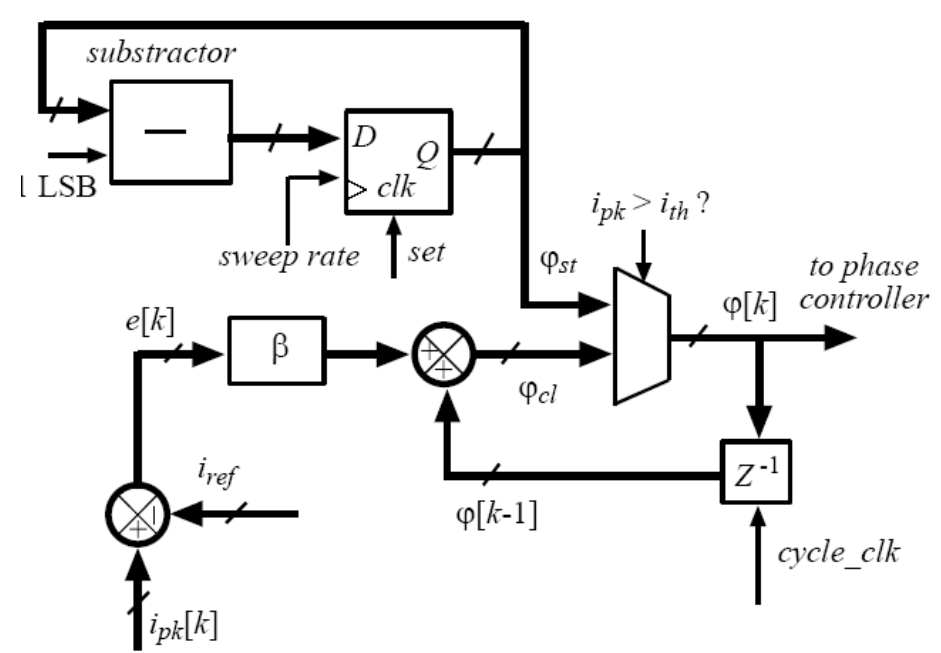

Figure 3-36. Block diagram of the startup controller combined with the compensator for smooth transitions between operating modes

This solution achieves a smooth transfer from phase sweep ignition to lamp current regulation modes by forcing the output of the compensator to be the same as the output of the phase sweep during startup.

\section{Analog Interface Blocks}

The core analog interface blocks are the current-mode ac buffer, current-mode comparator, and current-mode A/D converter. The current-mode ac buffer was used to provide direct sensing of both positive and negative voltages (either single-ended or differential) with only one or two external resistors, eliminating off-chip rectifying diodes and filtering circuitry. The basic buffer structure is shown in Figure 3-37, configured for a single-ended input. The buffer provides an output current proportional to the driving voltage and matched external resistors $\mathrm{R}$ (based on two operational transconductance amplifiers (OTAs)). The OTAs were implemented using folded cascode techniques. For the single-ended configuration, the relationship for ideal OTAs is

$$
i_{\text {out }}=i_{1}-i_{2}=-\frac{v_{\text {in }}}{R}
$$

\section{Equation 3-6}

For inductor current zero-crossing detection, a simple current-mode comparator follows the buffer. This comparator is based on the high speed current-mode approach. The output of the comparator is a square wave with a rising edge synchronous to the positive zero crossing of the inductor current waveform. This signal is used by the phase controller to determine the switching period. 


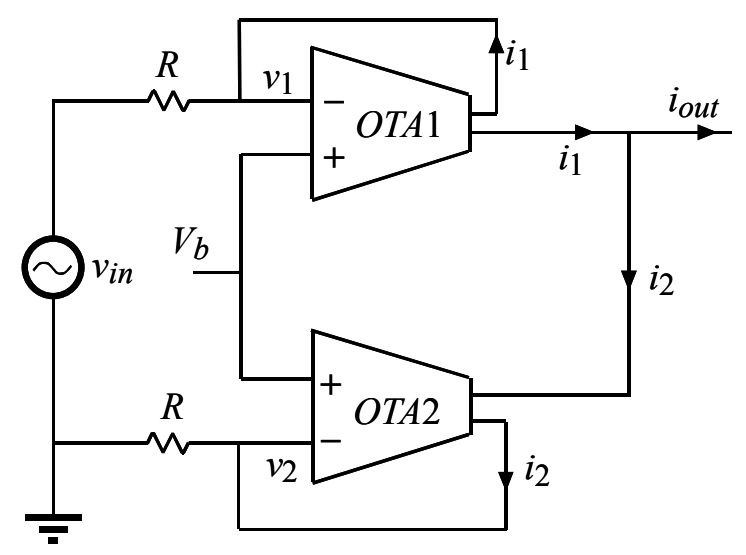

Figure 3-37. Analog ac buffer diagram

For lamp current and voltage A/D conversion, the output current is piped through a channel including analog current mode rectification (to simplify A/D), sample \& hold, and an asynchronous pipelined A/D converter, as shown in Figure 3-38.

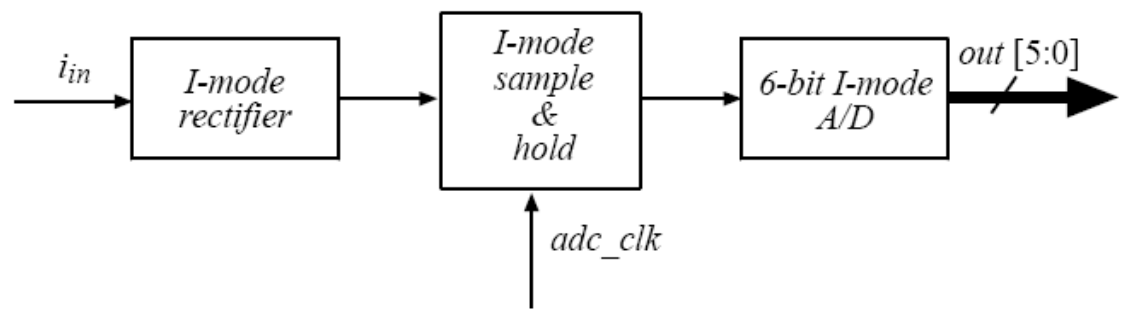

Figure 3-38. Block diagram for current-mode A/D conversion

A conceptual block diagram of the current-mode rectifier is shown in Figure 3-39. The currentmode sample \& hold circuit is based on a complete clock-feedthrough (CFT) cancellation approach. The S\&H block samples the full-wave rectified current with a sampling frequency of 16 or 32 times ballast frequency, based on the synchronized clock $A D C_{-}$clk from the phase controller. This ensures that exactly 16 or 32 samples of output waveforms are taken in each switching period, despite variations in switching frequency. 


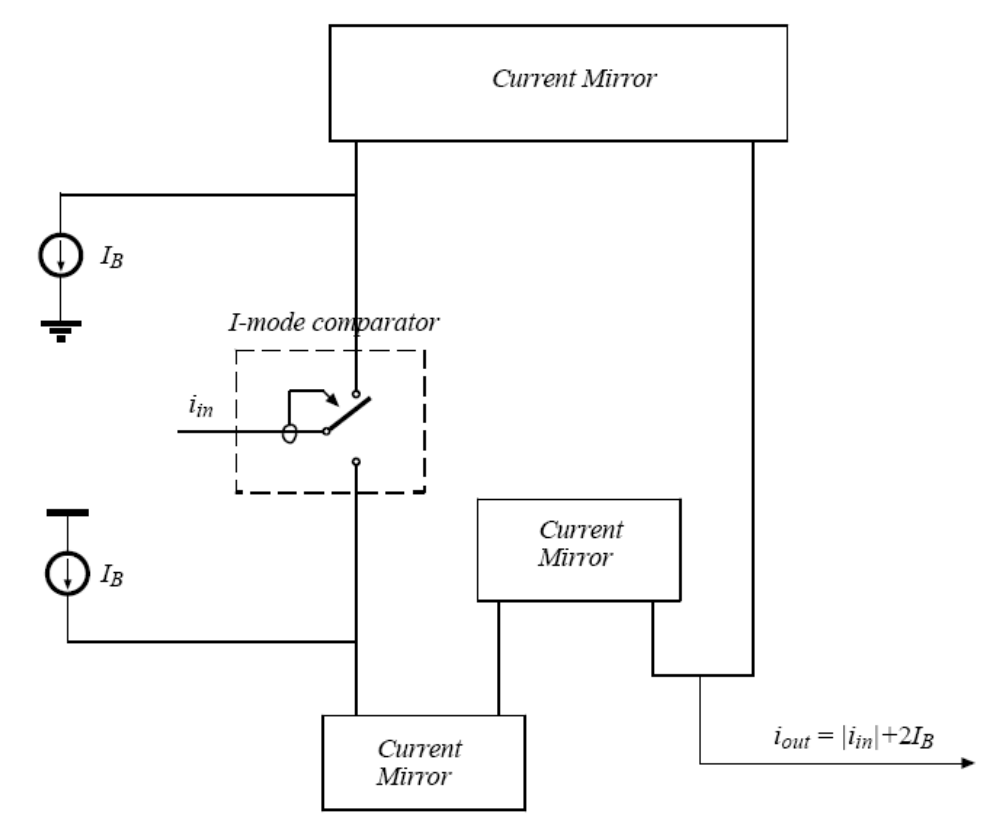

Figure 3-39. Simplified conceptual diagram of the current-mode rectifier

The 6-bit current-mode A/D is realized using an asynchronous pipelined structure, as shown in Figure 3-40 for the first two MSBs. The input current to each stage is compared to $1 / 2$ the reference current to get the digital output for that bit. If the input current is greater than $1 / 2$ the reference current, the bit will be on, and $1 / 2$ the reference current is subtracted from the input current, the result is multiplied by two, and then passed to the next stage. If the input current is less than $1 / 2$ the reference current, the bit will be off, and the input current is directly multiplied by two and then passed to the next stage.

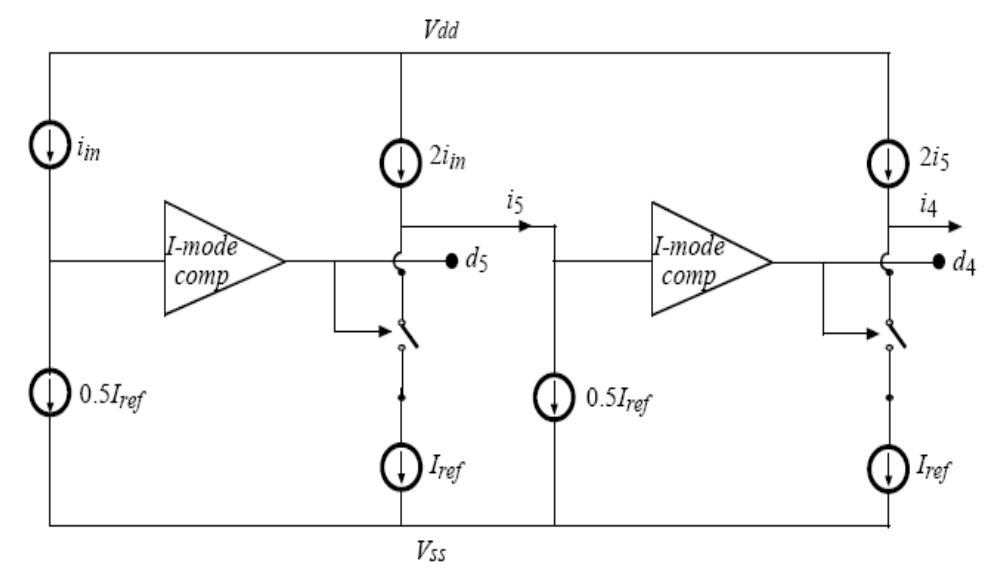

Figure 3-40. First two MSBs of the asynchronous pipe-lined A/D converter 


\subsubsection{FPGA-Based Verification of Ballast Controller}

An FPGA-based prototype is built to verify the key control functions of the digital controller. The diagram for the test-bed with a 400W HID ballast (resistive load) is illustrated in Figure 3-41. The digital control functions are implemented in a Virtex II xc2v1000 FPGA except that the over voltage protection is realized with the analog comparison instead of the digital comparison. The inductor current is sensed by a current transformer (CT) and then compared with zero potential through an off-chip comparator. The A/D converter is the THS 1230 from Texas Instruments, which is a 12-bit A/D with only eight bits being used for our application. As this $\mathrm{A} / \mathrm{D}$ converts the ac input, the effective resolution for the peak current is 7-bit. The phase resolution for the evaluation test-bed is 9-bit and the system clock is $200 \mathrm{MHz}$ with $5 \mathrm{~ns}$ time resolution. An external switch is used to generate the reset signal to emulate the on-chip reset from the power-on process.

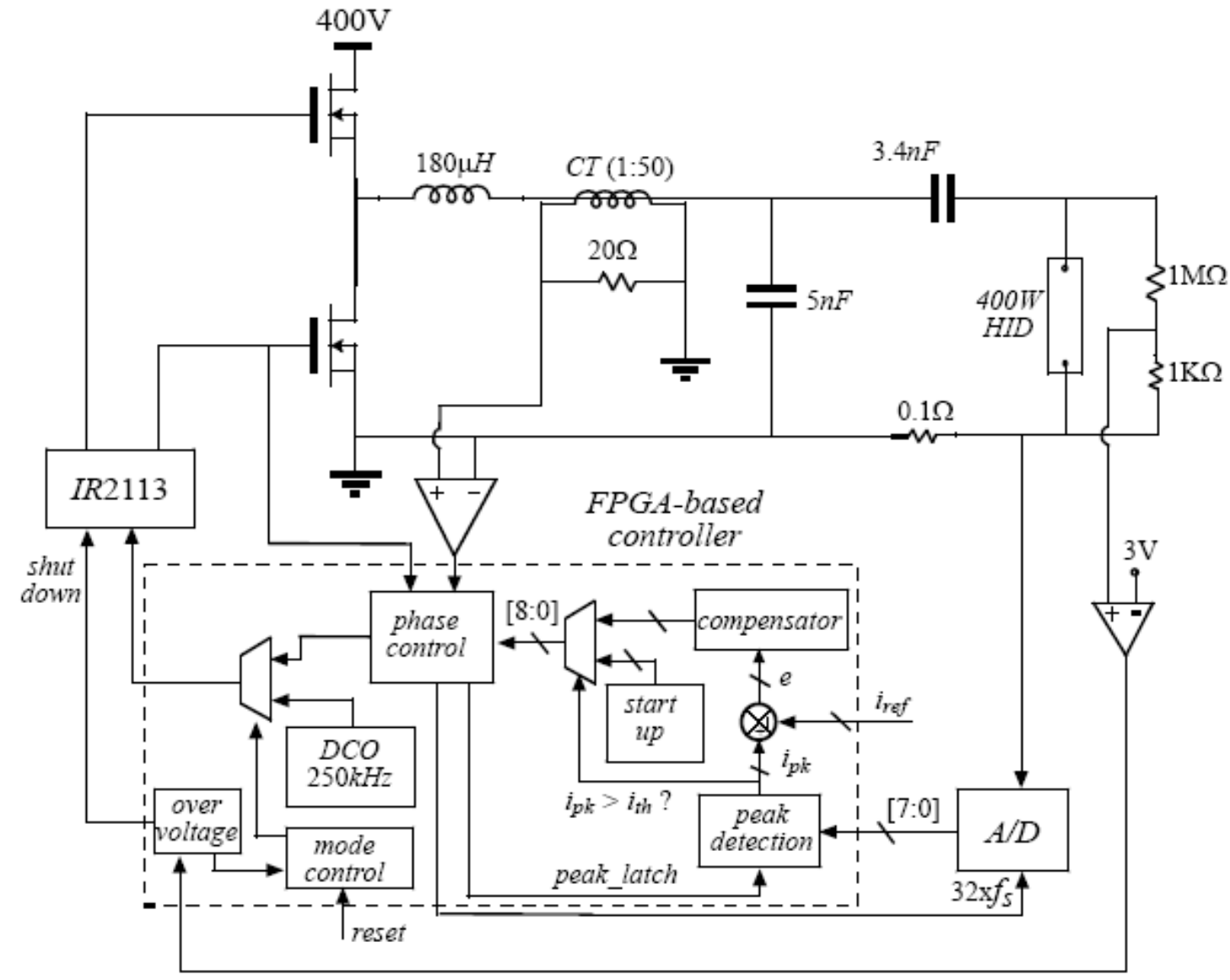

Figure 3-41. System diagram of FPGA-based prototype for ballast controller verification

When the system is reset manually by the external switch, it runs in the DCO mode for $10 \mathrm{~ms}$. The start-up controller then sweeps the phase to ignite the lamp. Once the lamp current exceeds the programmable current threshold, the system operates in the closed-loop mode to regulate the lamp current. The lamp voltage for the normal operation is about $200 \mathrm{~V}$ and the ignition voltage 
is about $1 \mathrm{kV}$. The over voltage threshold is set to $3 \mathrm{kV}$ that cannot be reached in the normal operation range. The full power operating frequency is $140 \mathrm{kHz}$.

The steady-state operation waveforms are given in Figure 3-42 and the step response is shown in Figure 3-43. It can be seen that the system is stable with a little bit of overshoot. Figure 3-44 illustrates the start-up process with a $40 \Omega$ resistive load. The three operation modes can be seen clearly and the mode transition from start-up to closed-loop is very smooth.

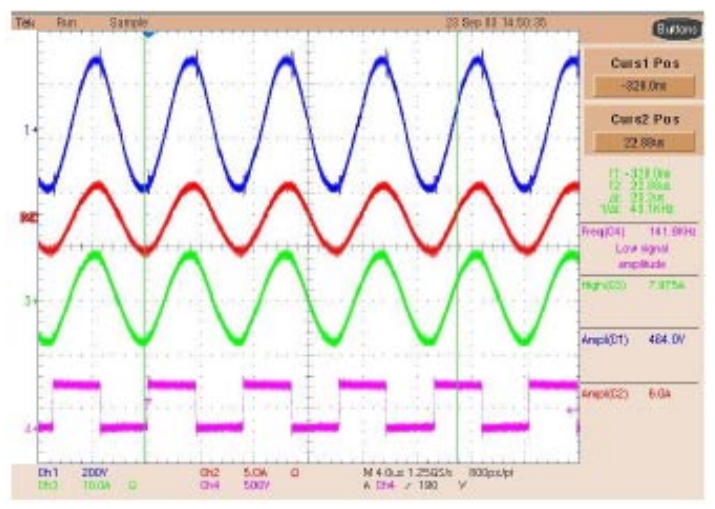

(a)

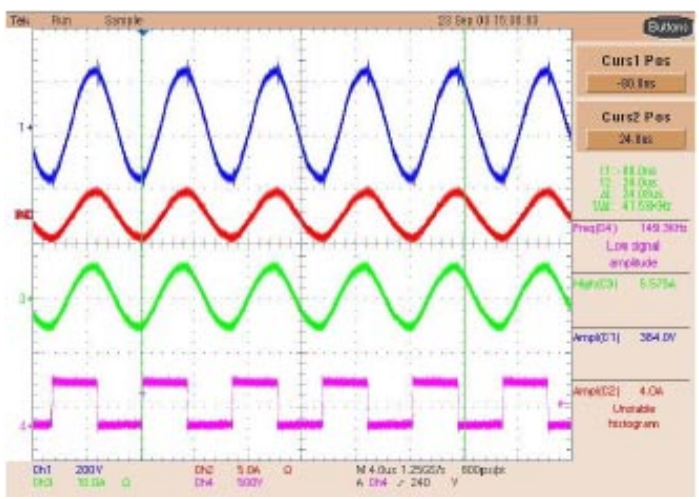

(b)

Figure 3-42. Steady-state waveforms for HID ballast with resistive load, ch1: lamp voltage; ch2: lamp current; ch3: inductor current; ch4: midpoint voltage; a) load current $=3 \mathrm{~A}$ peak, load power $=350 \mathrm{~W}$; b) load current $=2 \mathrm{~A}$ peak, load power $=190 \mathrm{~W}$

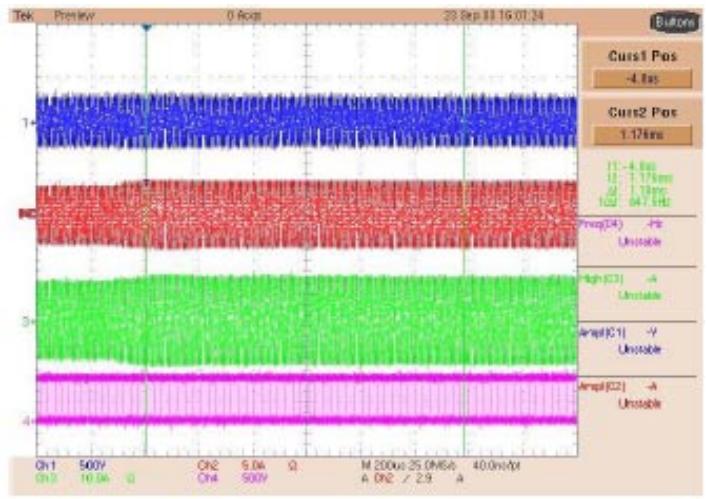

Figure 3-43. Step responses for HID ballast with resistive load: load current $=2.5 \mathrm{~A} \rightarrow 2.8 \mathrm{~A}$ peak, ch1: lamp voltage; ch2: lamp current; ch3: inductor current; ch4: mid-point voltage 


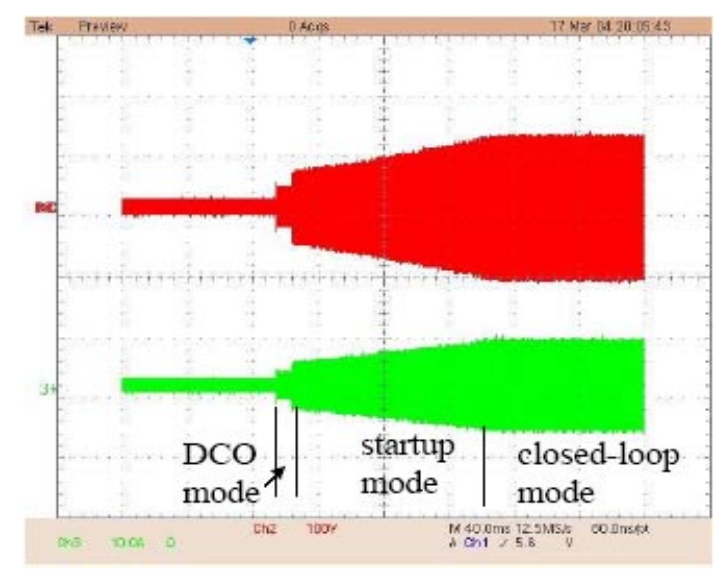

Figure 3-44. Start-up waveforms with resistive load, ch2: output voltage; ch3: inductor current

\subsubsection{Ballast Controller Custom IC Simulation Results}

The digital ballast controller is designed and implemented through the AMS $0.8 \mu \mathrm{m} 5 \mathrm{~V}$ CMOS process. Like the LVIC gate driver, the digital functions are described in Verilog and synthesized to the gate-level schematics through Cadence PKS tool. Figure 3-45 shows the layout of the IC with the dimension of $4.2 \mathrm{~mm} \times 3.2 \mathrm{~mm}$. About $85 \%$ of the die area is occupied by the digital circuitry. The chip resides in a 68-pin package with most of pins used for testing.

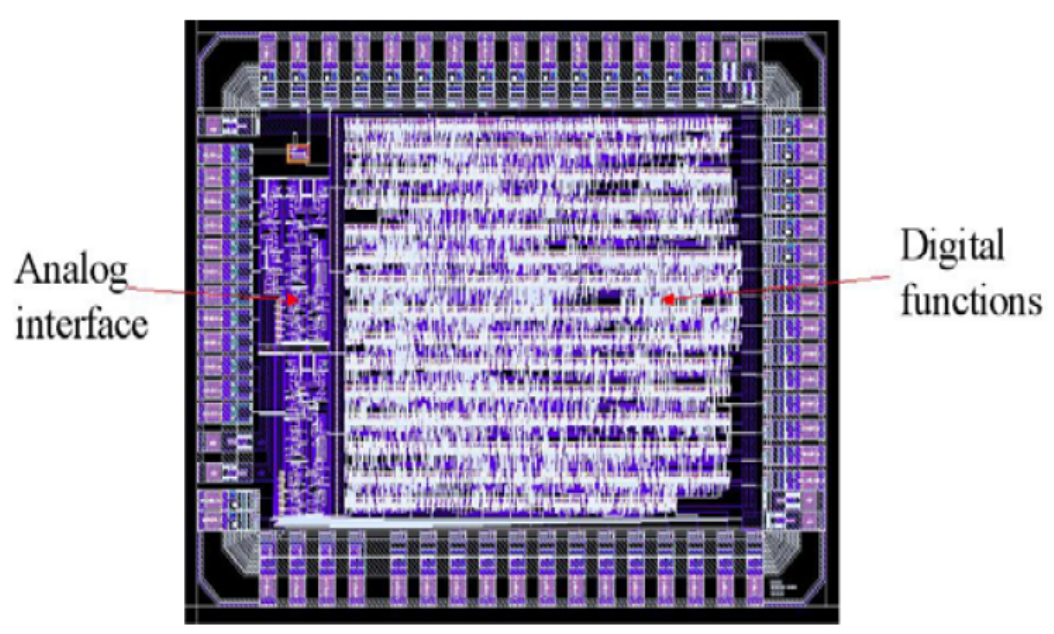

Figure 3-45. Photograph of the ballast controller, measuring $4.2 \mathrm{~mm} \times 3.2 \mathrm{~mm}$

The IC designs are verified through two approaches: the digital functions are examined by the FPGA prototype as demonstrated in Section 3.2.5; the analog designs are simulated in Cadence analog simulator. Key simulation results for the analog designs are presented. 


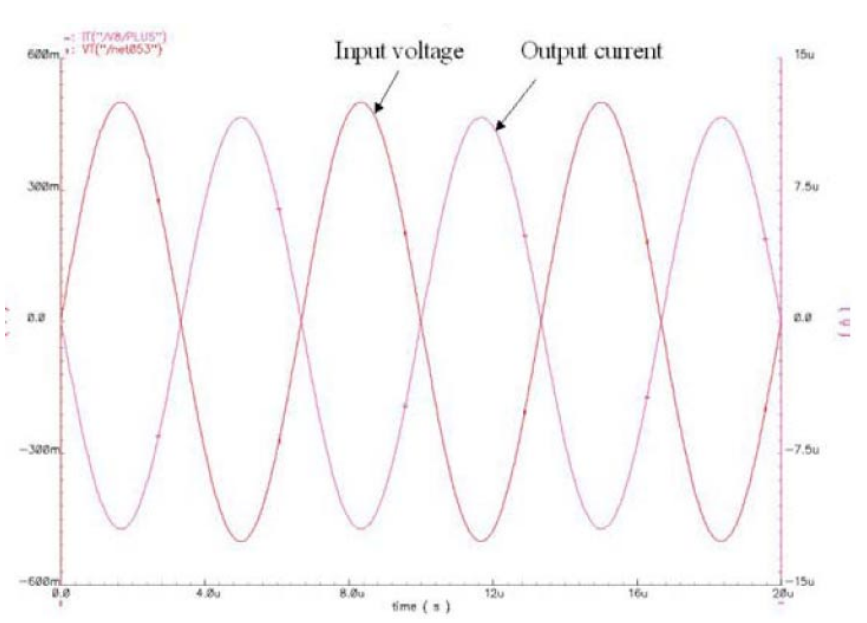

Figure 3-46. Simulation results for ac buffer (input ac voltage: $500 \mathrm{mV}$; output current: $11.85 \mu \mathrm{A}$ )

Figure 3-46 shows the simulation result for the ac buffer. The simulation is performed with the single-end input as configured in Figure 3-37 and the scale resistor is $40 \mathrm{k} \Omega$. The magnitude and frequency of the input voltage are $500 \mathrm{mV}$ and $150 \mathrm{kHz}$, respectively. The magnitude of the ideal output current, according to Equation 3-6, should be $12.5 \mu \mathrm{A}$. The simulation result is $11.85 \mu \mathrm{A}$ and the conversion error is about $5 \%$.

Figure 6.22 plots the simulation result for the I-mode rectifier. The magnitude of the input sinusoidal current is $25 \mu \mathrm{A}$ and the frequency is $150 \mathrm{kHz}$. It can be seen that the output of the rectifier is the full-wave rectified current with an offset $(12.5 \mu \mathrm{A})$. The dc offset is introduced to avoid the distortion around the zero-crossing of the input current. It is removed in the I-mode A/D converter. The simulation result for the sample and hold circuit is presented in Figure 6.23. The settling time is about 50ns and the clock-feedthrough error is about 100nA. Figure 6.24 shows the dc sweep simulation result for the 6-bit I-mode A/D converter. The input dc current sweeps from $12.5 \mu \mathrm{A}$ to $37.5 \mu \mathrm{A}$.

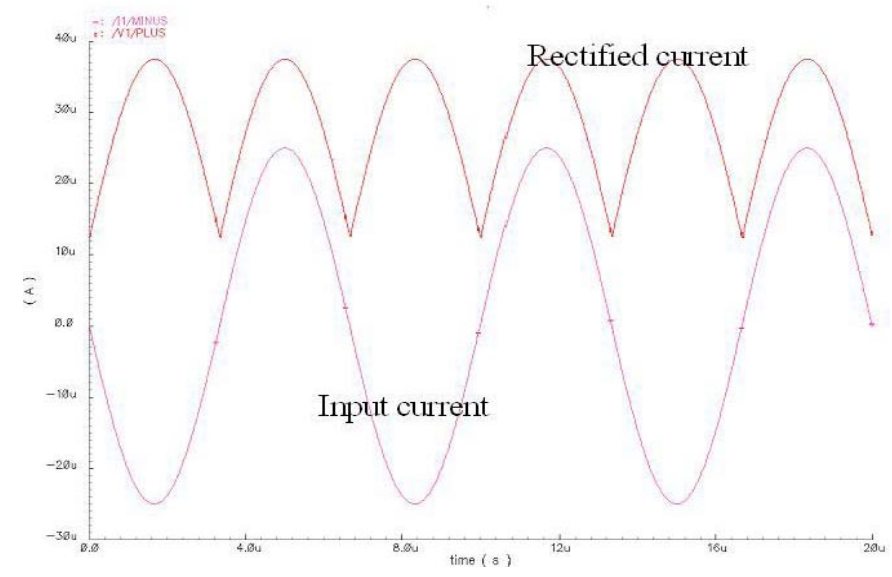

Figure 3-47. Simulation results for I-mode rectifier 


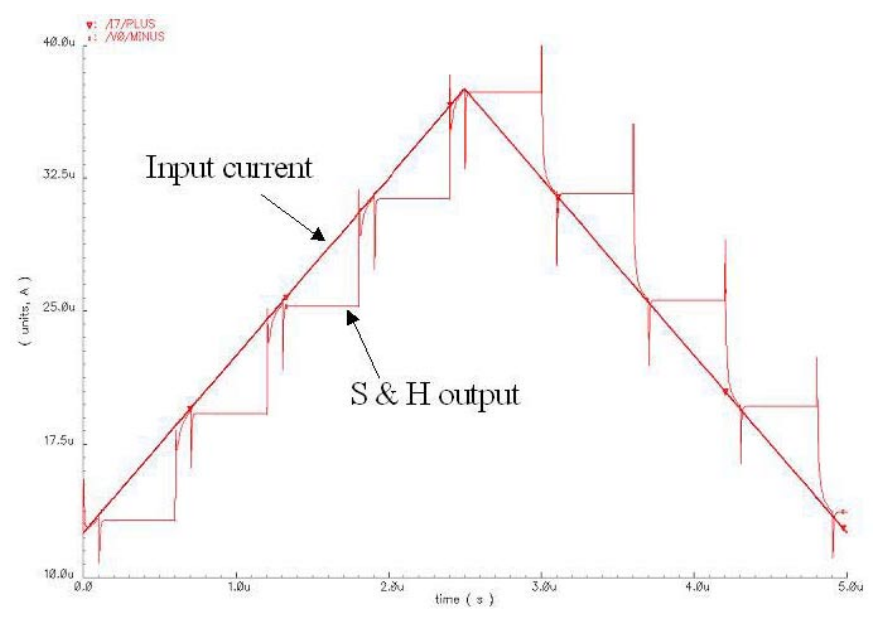

Figure 3-48. Simulation result for sample and hole circuit

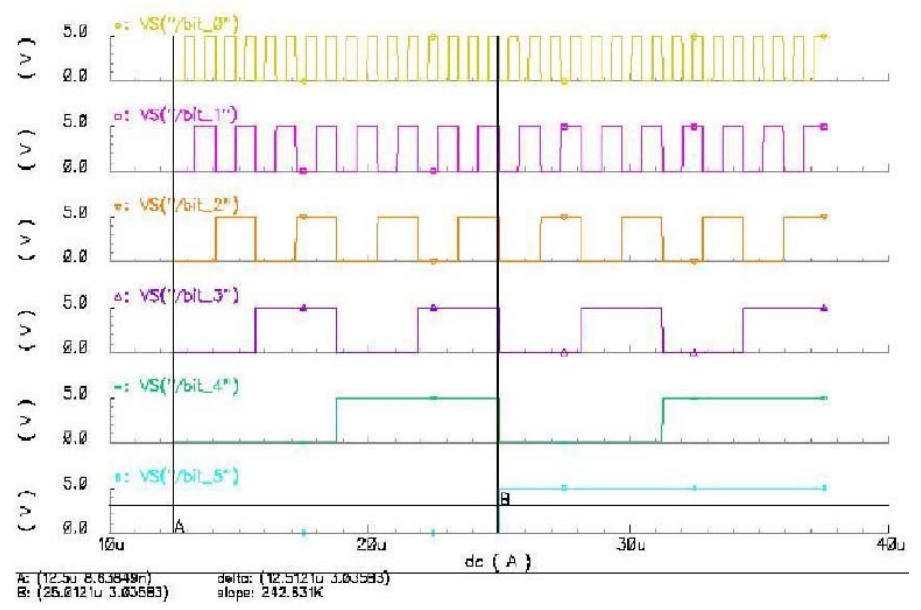

Figure 3-49. Simulation result for A/D converter by sweeping dc input current from $12.5 \mu \mathrm{A}$ to $37.5 \mu \mathrm{A}$

\subsection{Demo HID \& LFL Ballasts}

The LVIC gate drive is designed and fabricated through the AMS $0.8 \mu \mathrm{m} \mathrm{HV}(20 \mathrm{~V})$ process, and resides in 28-pin package with most of the pins used for testing. As shown in Figure 3-50(a) the chip dimension is about $2.2 \mathrm{~mm} \times 2.3 \mathrm{~mm}$. The on-chip CMOS switches are protected by the guard rings to limit the substrate current. The digital logic is also isolated by the guard ring to reduce the noises coupled from the analog circuitry.

The ballast controller IC is designed and implemented in a low-cost $0.8 \mu \mathrm{m}$ CMOS process, and resides in a 68-pin ceramic leadless package with most of pins used for testing. The chip 
dimension is about $4.2 \mathrm{~mm} \times 3.2 \mathrm{~mm}$, as shown in Figure $3-50(\mathrm{~b})$, with about $85 \%$ of the die area occupied by the digital circuitry.

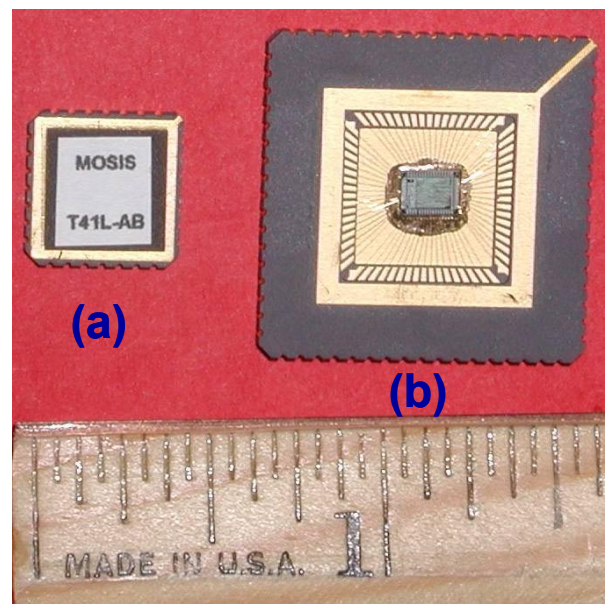

Figure 3-50. Prototype ICs: (a) gate driver IC in 28-pin package; (b) ballast controller IC in 68-pin package.

Two demo ballast prototypes were developed to test and validate the performance of the developed dual LVIC gate drive and ballast controller IC. As discussed previously, one of the objectives is to develop a universal control applicable for a wide range of lamp technologies. For demonstration purpose, HID and LFL ballasts were selected since the characteristics of HID and LFL lamps are significantly different from each other. As shown in Figure 3-51 and Figure 3-52 the only changes made to the controller IC were the lamp current and voltage references, which demonstrates that developed IC can be applied to different systems with minimum design changes. Please refer to the appendix for the detailed pin-out of the IC chip set and schematics of the demo ballast. 


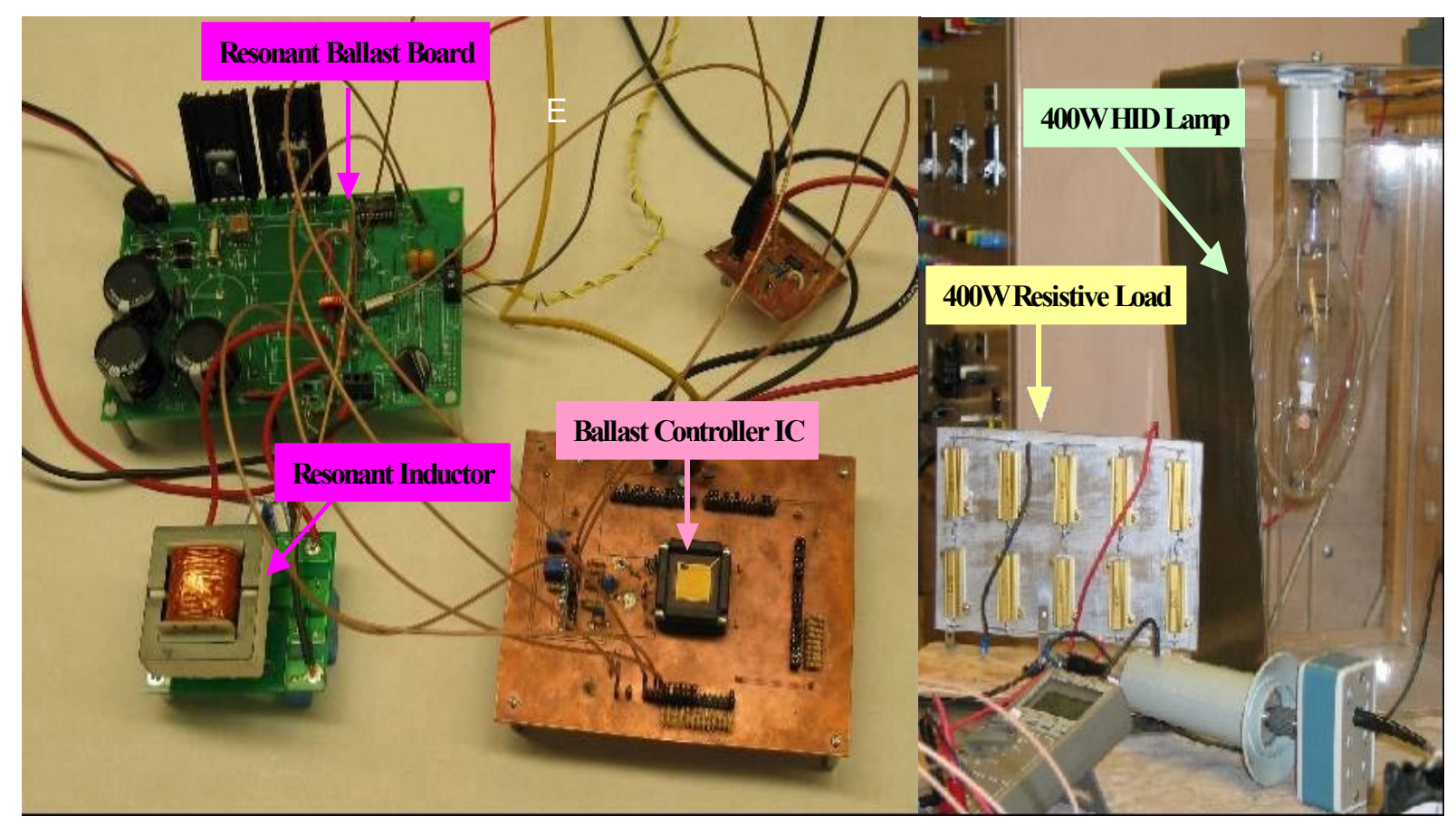

Figure 3-51. 400W HID demo ballast prototype

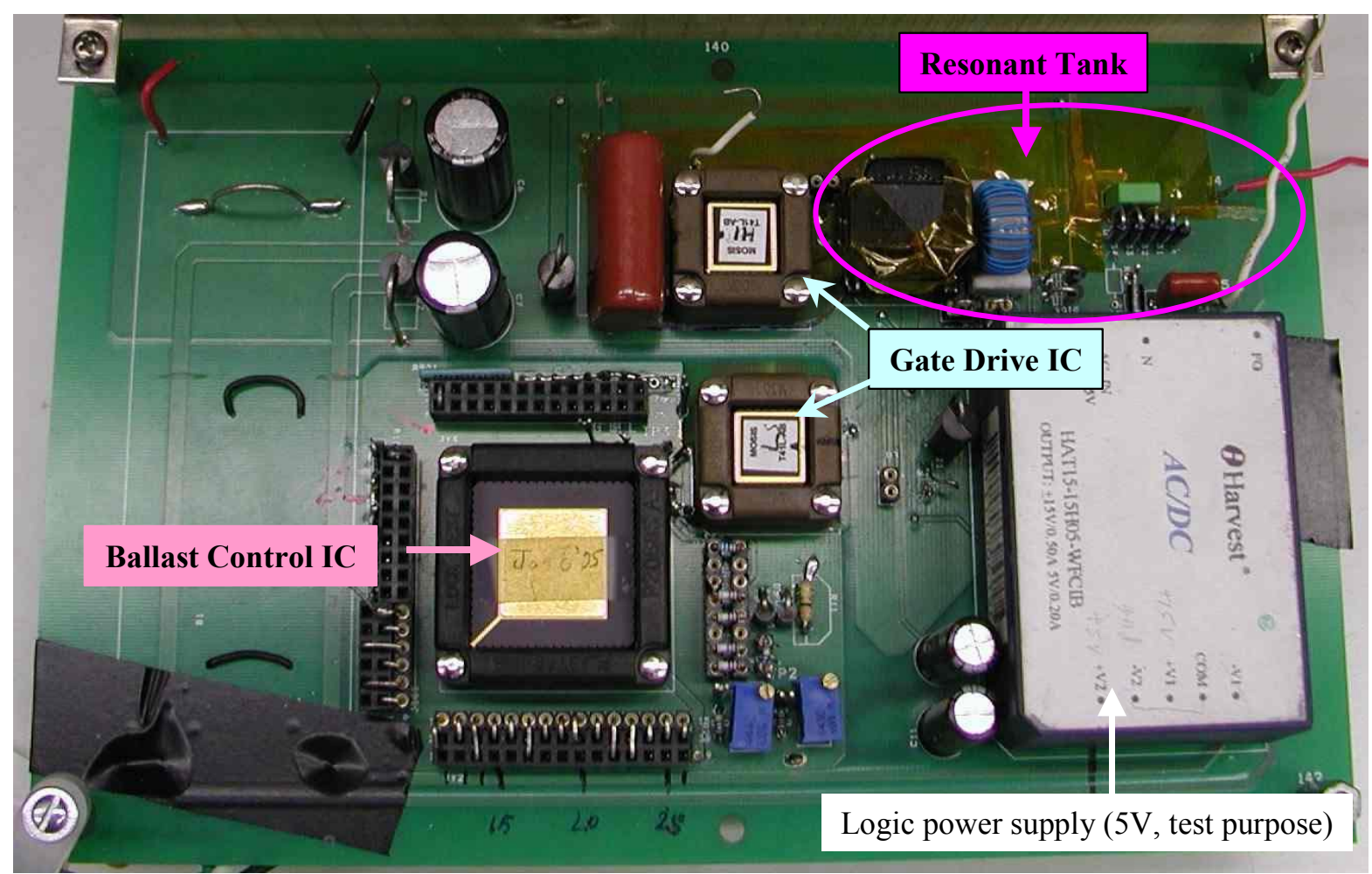

Figure 3-52. 32W LFL demo ballast prototype 


\section{EXPERIMENTAL METHODS}

During the course of the research simulation tools and FPGA prototype tools were used extensively for process and temperature corner examination and rapid prototyping with realistic hardware realization before IC layout design. The design is verified through simulation of each functional block as well as the whole system. The digital functions are examined based on the Insight/Memec development board for the Xilinx Virtex II FPGA and analog designs are simulated in Cadence analog simulator.

During the IC prototype and demo ballast debug period, following equipments were used:

- Agilent 6813A AC Power Source for ballast power (the DC offset of the power source was used to provide DC power with the AC magnitude set to zero);

- BK Precision 1670 DC Power Supply for 12 V and 5 V control power;

- Tektronix TDS 3012B Digital Oscilloscope for 2-channel analog waveforms;

- Tektronix TDS 7054 Deep Memory Digital Oscilloscope for 4-channel analog waveforms and transient phenomena investigation;

- Hewlett Packard 1661 CS Logic Analyzer for digital signals;

- Harrison Laboratories 855C Power Supply for A/D testing;

- Agilent 33120 Function Generator to provide external A/D clock for A/D testing; 


\section{RESULTS AND DISCUSSION}

The IC prototype tests include 1) dual LVIC gate deriver test, b) ballast controller IC test, and c) demo ballast test with gate driver IC and ballast controller IC.

\subsection{LVIC Gate Driver Test}

The testing of the gate driver IC prototype is mainly involved into the performance evaluation on three major categories: 1) charge-pump operation for low and high side power supply; 2) analog performance for signaling and handshaking pulse generation and detection, and high or low side gate driving; 3) control logic function for the generation of capacitor and gate driving signals, timing constant, fault protection, and dual-IC mode / floating mode selection. The auxiliary circuits are necessary for the IC operation and should be tested first.

\subsubsection{Auxiliary Circuit Test (Bias and Clock Generation Blocks)}

1) Bias block:

Current bias is used for current reference in detectors and bias for clock generation. It is programmable through $f_{-} s e l$ with the expected values of $\{21.25 \mathrm{uA}, 23.75 \mathrm{uA}, 26.25 \mathrm{uA}$, $28.75 \mathrm{uA}\} \pm 22 \%$.

a. Verify current bias at different $f \_$sel settings:

i. Tie bias (pin 1) to gnd! through $210 \mathrm{k} \Omega$ resistor, measure voltage across pin 1.

Table 5-1. Test results of current bias at different f_sel settings:

\begin{tabular}{|c|c|c|c|c|c|c|c|c|c|c|c|}
\hline \multirow{2}{*}{$\begin{array}{l}\mathrm{f} \_ \text {sel } \\
\text { setting }\end{array}$} & \multicolumn{3}{|c|}{ Voltage@pin 1} & \multicolumn{3}{|c|}{ Calculated $\mathrm{I}(\mu \mathrm{A})$} & \multirow{2}{*}{$\begin{array}{l}\text { Expected bias I } \\
\qquad(\mu \mathrm{A})\end{array}$} & \multicolumn{4}{|c|}{ Error $(\%)$} \\
\hline & $\# 1$ & $\# 2$ & $\# 3$ & $\# 1$ & $\# 2$ & $\# 3$ & & $\# 1$ & $\# 2$ & $\# 3$ & Ave \\
\hline 00 & 9.18 & 9.06 & 8.72 & 43.7 & 43.1 & 41.5 & 28.75 & 52 & 50 & 44 & 49 \\
\hline 01 & 8.57 & 8.30 & 7.99 & 40.8 & 39.5 & 38.0 & 26.25 & 55 & 51 & 45 & 50 \\
\hline 10 & 7.93 & 7.64 & 7.24 & 37.8 & 36.4 & 34.5 & & 59 & 53 & 45 & 52 \\
\hline 11 & 7.28 & 7.04 & 6.75 & 34.7 & 33.5 & 32.1 & 21.25 & 63 & 58 & 51 & 57 \\
\hline
\end{tabular}

Discussion: This test verifies the bias block's ability to generate different currents depending on the $f_{-}$sel setting. However, the bias currents are about 53\% (average) greater than the expected values. The spread among chips was small, within a few percent of each other. The current shiftup will increase the threshold of the current detection circuit.

b. Verify bias current for power supply vddg_ $12>7 \mathrm{~V}\left(@ f_{\_}\right.$sel $\left.=11\right)$

i. Tie bias (pin 1) to gnd! through $210 \mathrm{k} \Omega$ resistor, measure voltage across pin 1;

ii. Sweep $12 \mathrm{~V}$ supply voltage down to zero; 
Table 5-2. Test results of current bias at different supply voltages:

\begin{tabular}{|c|c|c|c|c|c|c|}
\hline \multirow{2}{*}{ Supply voltage (V) } & \multicolumn{3}{|c|}{ Voltage @ pin 1 } & \multicolumn{3}{c|}{ Calculated I $(\mu \mathrm{A})$} \\
\cline { 2 - 7 } & $\# 1$ & $\# 2$ & $\# 3$ & $\# 1$ & $\# 2$ & $\# 3$ \\
\hline 12 & 7.46 & 7.04 & 6.87 & 35.5 & 33.5 & 32.7 \\
\hline 10 & 7.29 & 7.01 & 6.85 & 34.7 & 33.4 & 32.6 \\
\hline 8 & 6.34 & 6.36 & 6.32 & 30.2 & 30.3 & 30.1 \\
\hline 7 & 5.65 & 5.69 & 5.69 & 26.9 & 27.1 & 27.1 \\
\hline 6 & 4.91 & 4.96 & 4.91 & 23.4 & 23.6 & 23.4 \\
\hline
\end{tabular}

Discussion: This test verifies that the bias block can supply the correct current levels, even at voltages lower than $12 \mathrm{~V}$. The results show that all chips drop the current to $30 \mathrm{uA}$ at $8 \mathrm{~V}$ and to $27 \mathrm{uA}$ at $7 \mathrm{~V}$, regardless of the current supplied at a full $12 \mathrm{~V}$. The chips exhibit current drops of $15 \%, 20 \%$ and $18 \%$, respectively, when moving from $12 \mathrm{~V}$ to $7 \mathrm{~V}$.

c. Verify on-chip 5V linear voltage regulator to be $5 \mathrm{~V}$ and capable to produce $2 \mathrm{~mA}$ current: i. $\quad$ Tie a variable resistor to vdd! (pin 15);

ii. Measure the current drawn from vdd! until vdd! changes by $10 \%$.

Table 5-3. Test results of $5 \mathrm{~V}$ linear voltage regulator:

\begin{tabular}{|c|c|c|c|c|c|}
\hline \multicolumn{2}{|c|}{ Voltage @ vdd! (pin 15) (V) } & \multicolumn{3}{c|}{ Current from vdd! (pin 15) (mA) } \\
\hline$\# 1$ & $\# 2$ & $\# 3$ & $\# 1$ & $\# 2$ & $\# 3$ \\
\hline 6.33 & 5.95 & 5.96 & .5 & .5 & .5 \\
\hline 6.33 & 5.95 & 5.96 & 1 & 1 & 1 \\
\hline 6.33 & 5.95 & 5.95 & 1.5 & 1.5 & 1.5 \\
\hline 6.33 & 5.95 & 5.95 & 2 & 2 & 2 \\
\hline 6.04 & 5.84 & 5.84 & 8 & 8 & 8 \\
\hline 5.70 & 5.34 & 5.34 & 10.5 & 12.2 & 12.2 \\
\hline
\end{tabular}

Discussion: This test shows that the $5 \mathrm{~V}$ supply that the chip produces is about $20 \%$ greater than the expected $5 \mathrm{~V}$ and is able to produce $\sim 12 \mathrm{~mA}$ current, well above the requirement up $(\sim 510 \%$ more) before dropping the voltage by more than $10 \%$.

2) Clock generation block:

i. Tie pin 11 through a $10 \mathrm{k} \Omega$ pull-up resistor to $5 \mathrm{~V}$, measure frequency;

ii. Record frequencies for all $f$ sel settings.

Table 5-4. Test results of clock generation:

\begin{tabular}{|c|c|c|c|}
\hline \multirow{2}{*}{ f_sel setting } & \multicolumn{3}{|c|}{ Frequency $(\mathrm{kHz})$} \\
\cline { 2 - 4 } & $\# 1$ & $\# 2$ & $\# 3$ \\
\hline 00 & 11.93 & 12.26 & 11.72 \\
\hline 01 & 10.92 & 11.18 & 10.76 \\
\hline
\end{tabular}




\begin{tabular}{|l|c|c|c|}
\hline 10 & 10.09 & 10.33 & 9.81 \\
\hline 11 & 9.32 & 9.56 & 9.20 \\
\hline
\end{tabular}

Discussion: This test verifies that the on-chip clock generator produces the correct frequencies at each of the $f$ sel settings. The test showed that at the bias setting closest to $25 \mathrm{uA}$, all chips were well within the $20 \%$ expected distribution. The other settings also show the expected trend of decreasing clock frequencies as $f \_$sel was increased to 11 .

\subsubsection{Charge Pump-Based Low and High Side Power Supply Test}

Half-bridge LFL ballast with resistive load was used to test the HS and LS power supply under $200 \mathrm{~V}$ bus voltage. As shown in Figure 5-1 sufficient energy is passed through 320pFcoupling capacitor in each cycle to power up LS as well as HS ICs.

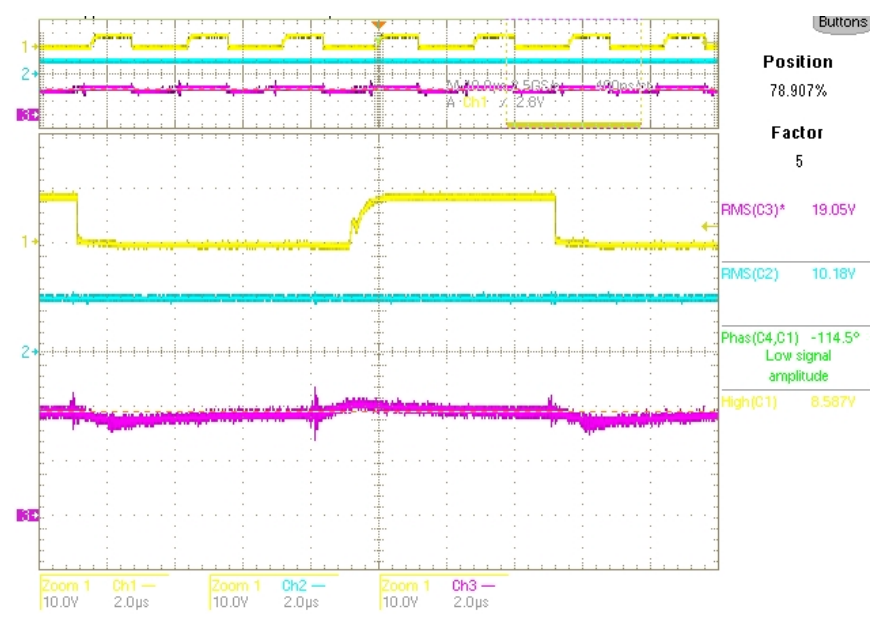

Figure 5-1. Test results demonstrating charge-pump operation for LS \& HS supply voltages

\subsubsection{Analog Circuitry Performance}

Analog performance testing consists of two parts: verifying signaling and handshaking pulse generation/detection and verifying gate driving capability.

1) Test of signaling and handshaking pulse generation:

a. Signaling pulse generation: signaling pulse is generated through low side cap switch (nchannel or p-channel MOSFETs), which is controlled by external signal, gd_ctrl.

i. $\quad$ Set hl_sel (pin 18) to low $0 \mathrm{~V}$ as low side mode, all others to zero;

ii. Set gd_ctrl (pin 14) to high 5V or low, verify the initial state of v_cap_in (pin 8) to be low and cap (pin 5) to be high (= vddc_12) after bringing up power; 
iii. Set gd_ctrl to high after initial pulse, verify v_cap_in is low and cap is high, repeat for gd_ctrl to low.

Table 5-5. Test results of cap switch logic:

\begin{tabular}{|c|c|c|c|c|}
\hline \multirow{2}{*}{ gd_ctrl (pin 14) } & \multicolumn{2}{|c|}{ initial setting } & \multicolumn{2}{c|}{ after initial pulse } \\
\cline { 2 - 5 } & $1(5 \mathrm{~V})$ & $0(0 \mathrm{~V})$ & $1(5 \mathrm{~V})$ & $0(0 \mathrm{~V})$ \\
\hline V_cap_in (pin 8) & $0(0 \mathrm{~V})$ & $0(0 \mathrm{~V})$ & $0(0 \mathrm{~V})$ & $1(5 \mathrm{~V})$ \\
\hline cap (pin 5) & $1(12 \mathrm{~V})$ & $1(12 \mathrm{~V})$ & $1(12 \mathrm{~V})$ & $0(0 \mathrm{~V})$ \\
\hline
\end{tabular}

Discussion: This test verifies the correct operation of the cap switches shown in Figure 3-18.

b. Handshaking pulse generation: handshaking pulse is generated through high side cap switch (n-channel or p-channel MOSFET), which is controlled by high side statemachine. The results are shown in section 5.1.4.

2) Test of signaling and handshaking pulse detection:

As shown in Figure 3-3 the HSIC needs to detect signaling currents and the LSIC needs to detect handshaking currents. When in HSIC mode detector senses negative current with an expected threshold of $25 \mu \mathrm{A}$, or $-15 \mathrm{~mA}$ cap switch input current, and in LSIC mode detector senses positive current with a threshold of $125 \mu \mathrm{A}$, or, $+45 \mathrm{~mA}$ cap switch input current. In the HSIC mode, the cap switch is driven by the state-machine, as shown in Figure 3-14 (b). The test of signaling detection can be verified in section 5.1.4.

a. N-channel current detection (static current input):

i. Set gb_ctrl (pin 14) to low 0V and hl_sel (pin 18) to low;

ii. Tie i_ndet (pin 2) to gnd! through a $20 \mathrm{k} \Omega$ resistor;

iii. Tie $\mathrm{v} \_$ndet (pin 9) to $5 \mathrm{~V}$ through a $5 \mathrm{k} \Omega$ resistor;

iv. Inject $0 \sim 100 \mathrm{~mA}$ current into cap (pin 5) from a voltage source through a current limit resistor, monitor pin $2 \& 9$. Expected current flowing out of pin 2 equals $\mathrm{i}_{\text {in }} / 600+50 \mu \mathrm{A}$.

Table 5-6. Test results of threshold current of n-channel current detector:

\begin{tabular}{|c|c|c|c|c|c|c|c|c|c|c|c|c|c|}
\hline \multirow{2}{*}{$\begin{array}{c}\mathrm{i}_{\text {in }} \\
(\mathrm{mA})\end{array}$} & \multicolumn{3}{|c|}{$\begin{array}{l}\text { i ndet (V) } \\
(\mathrm{V} \text { in pin } 2)\end{array}$} & \multicolumn{3}{|c|}{$\begin{array}{l}\text { i_ndet }(\mu \mathrm{A}) \\
\text { (cal. I from } \mathrm{V})\end{array}$} & \multirow[t]{2}{*}{$\begin{array}{l}\text { Exp } \\
\text { i_ndet } \\
(\mu \mathrm{A})\end{array}$} & \multicolumn{3}{|c|}{ Error \% } & \multicolumn{3}{|c|}{ v_ndet (pin 9) } \\
\hline & $\# 1$ & $\# 2$ & $\# 3$ & $\# 1$ & $\# 2$ & $\# 3$ & & $\# 1$ & $\# 2$ & $\# 3$ & $\# 1$ & $\# 2$ & $\# 3$ \\
\hline 100 & 4.76 & 4.85 & 4.77 & 238 & 242.5 & 238.5 & 217 & 10 & 12 & 10 & 1 & 1 & 1 \\
\hline 80 & 4.20 & 4.12 & 4.04 & 210 & 206 & 202 & 183 & 15 & 12 & 10 & 1 & 1 & 1 \\
\hline 60 & 3.46 & 3.45 & 3.34 & 173 & 172.5 & 167 & 150 & 15 & 15 & 11 & 0 & 1 & 0 \\
\hline 40 & 2.76 & 2.69 & 2.59 & 138 & 134.5 & 129.5 & 117 & 18 & 15 & 11 & 0 & 0 & 0 \\
\hline 20 & 2.02 & 1.97 & 1.87 & 101 & 98.5 & 93.5 & 83 & 21 & 18 & 12 & 0 & 0 & 0 \\
\hline \multicolumn{4}{|c|}{ Measured threshold (mA) } & 67 & 59.6 & 62.8 & & & & & & & \\
\hline
\end{tabular}


Discussion: All three chips showed functioning n-channel comparators and detectors with a small amount of spread. The comparator switching thresholds were offset by about $40 \%$ from the expected $45 \mathrm{~mA}$ threshold due to $\sim 50 \%$ shift-up current bias in the current bias test. The i_ndet data is very close to the expected $\mathrm{i}_{\mathrm{in}} / 600+50 \mu \mathrm{A}$ but actually has a scale factor closer to 500 , as shown in Figure 5-2.

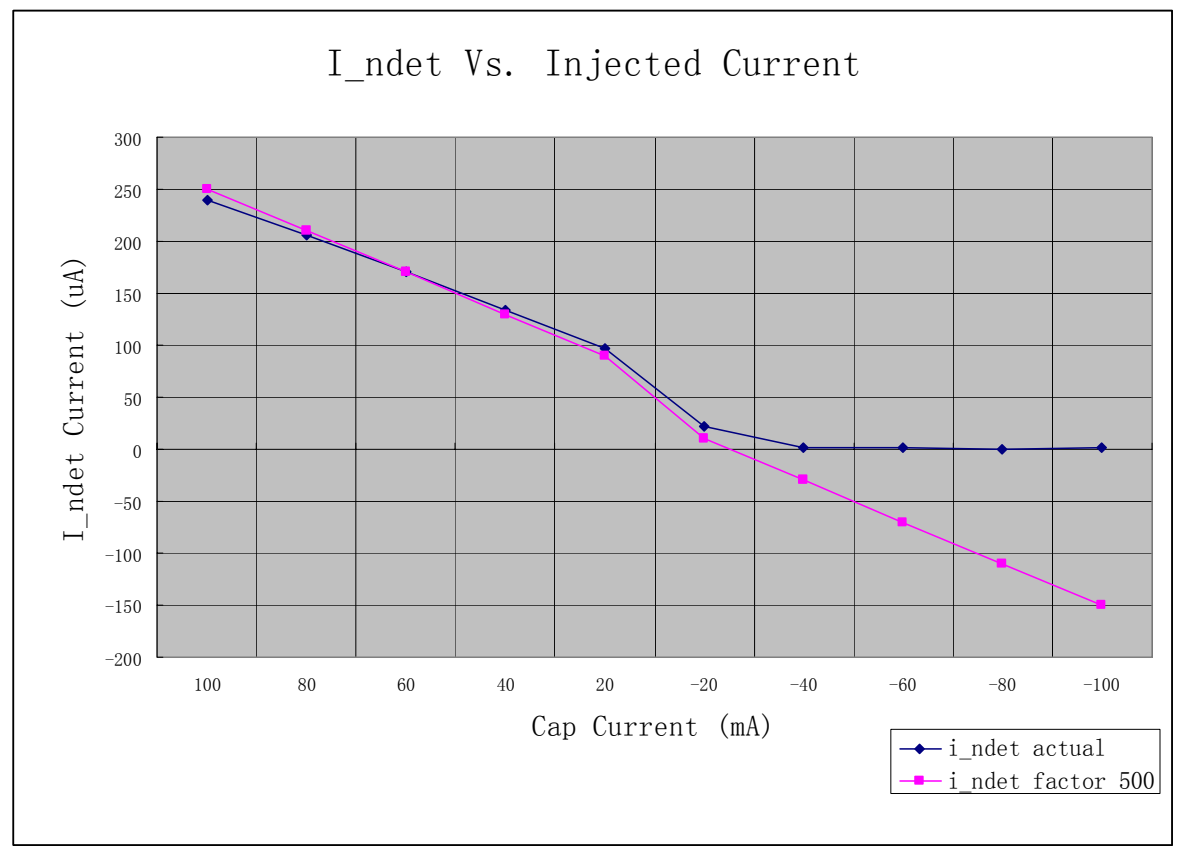

Figure 5-2. Current detector output current, a) measured, b) calculated with a factor of 500

b. N-channel current detection (switching mode):

This test is similar to the previous one, except that the injected current is a high frequency $(\sim 100 \mathrm{kHz})$ current waveform to test the ability of scaling and tracking currents in the cap switch. 


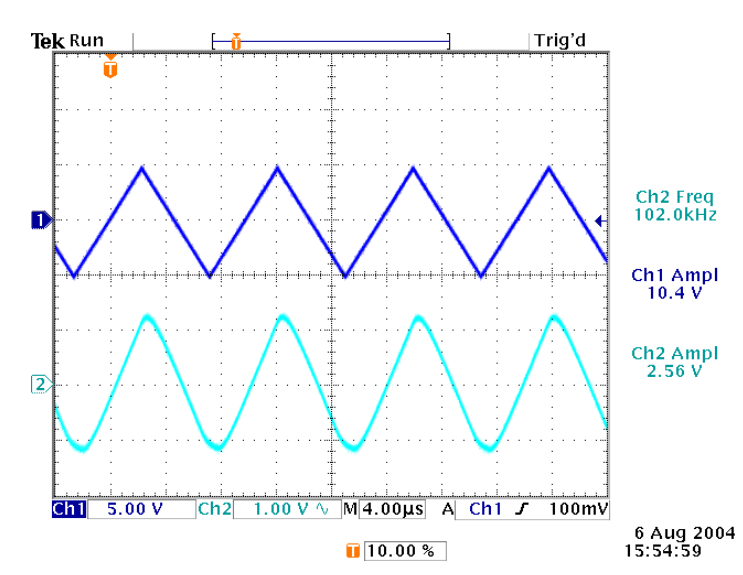

(a)

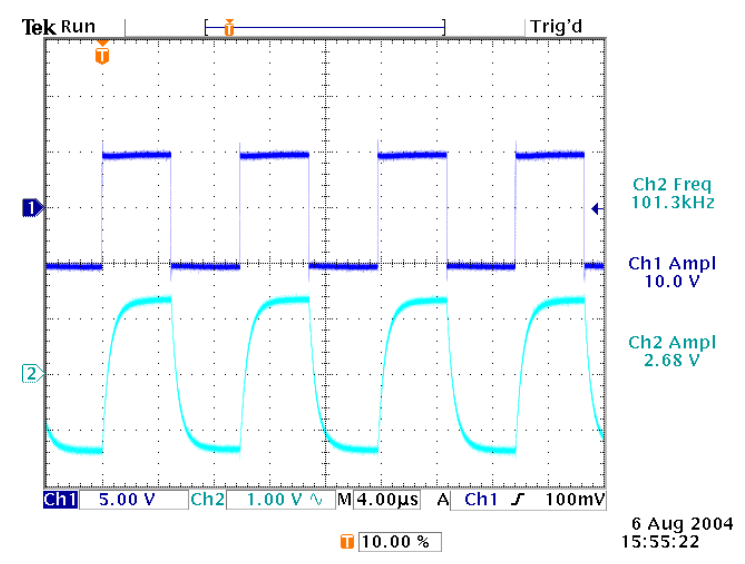

(b)

Figure 5-3. Experimental results demonstrating current scaling and detection operations for $\pm 100 \mathrm{~mA}$ injection for a) triangle waveform, and b) square waveform; ch1: injection current to cap pin $50 \mathrm{~mA} / \mathrm{dov}$, ch2: current detector output $50 \mu \mathrm{A} / \mathrm{div}$

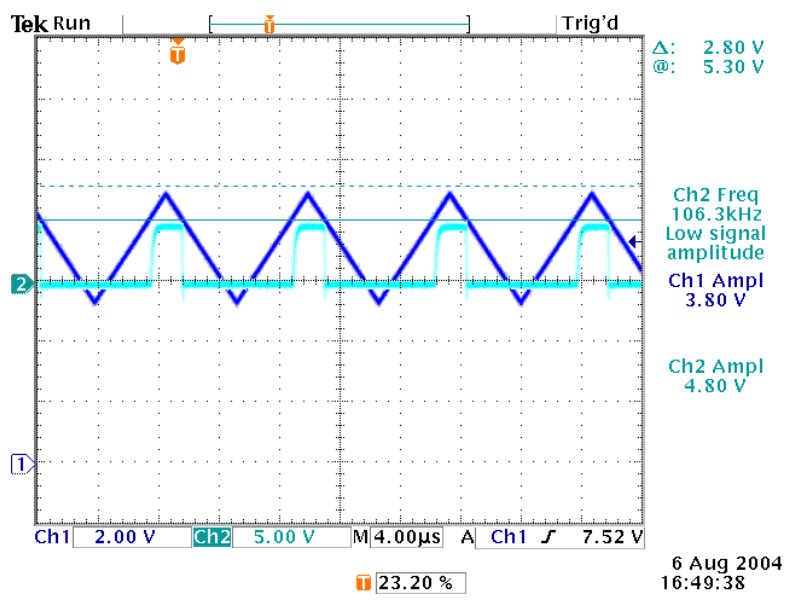

Figure 5-4. Experimental results demonstrating current comparator operation with a $+68 \mathrm{~mA}$ threshold, ch1: injection current $20 \mathrm{~mA} /$ div, ch2: current comparator output

Discussion: Figure 5-3 show proper operation for scaling and tracking large positive and negative currents. Figure 5-4 shows correct operation of the current comparator with a shift-up threshold of $68 \mathrm{~mA}$.

c. P-channel current detection:

The test is similar to the n-channel current detection. One chip was tested.

i. Set gb_ctrl (pin 14) to high 5V after a initial pulse, and hl_sel (pin 18) to low;

ii. Tie i_pdet (pin 3) to vddg_12 through a $20 \mathrm{k} \Omega$ resistor;

iii. Tie v_pdet (pin 10 ) to $5 \mathrm{~V}$ through a $5 \mathrm{k} \Omega$ resistor;

iv. Pull $0 \sim-100 \mathrm{~mA}$ current from cap (pin 5) through a current limit resistor, monitor pin $3 \& 10$. Expected current flowing out of pin 2 equals $\mathrm{i}_{\mathrm{in}} / 600+50 \mu \mathrm{A}$. 
Table 5-7. Test results of threshold current of p-channel current detector:

\begin{tabular}{|c|c|c|c|c|c|}
\hline $\begin{array}{c}\mathrm{i}_{\text {in }} \\
(\mathrm{mA})\end{array}$ & $\begin{array}{c}\text { i_pdet }(\mathrm{V}) \\
(\mathrm{V} \text { in pin 3) }\end{array}$ & $\begin{array}{c}\text { i_pdet }(\mu \mathrm{A}) \\
(\text { cal. I from V) }\end{array}$ & $\begin{array}{c}\text { Exp } \\
\text { i_pdet } \\
(\mu \mathrm{A})\end{array}$ & Error \% & v_pdet (pin 10) \\
\hline-20 & 9.96 & 102 & 83 & 22 & 0 \\
\hline-33.84 & 9.6 & 120 & 106 & 13 & 0 \\
\hline-20 & 9.97 & 101.5 & 83 & 22 & 0 \\
\hline-40 & 9.45 & 127.5 & 117 & 9 & 0 \\
\hline-60 & 8.94 & 153 & 150 & 2 & 0 \\
\hline-63.8 & 8.84 & 158 & 156 & 1 & Tran. (2.58V) \\
\hline-73 & 8.61 & 169.5 & 172 & -1 & 1 \\
\hline-88 & 8.23 & 188.5 & 197 & -4 & 1 \\
\hline \multicolumn{5}{|c|}{ Measured threshold (mA) } & \multicolumn{5}{|c|}{$63.8 \mathrm{~mA}$} & \\
\hline
\end{tabular}

Discussion: This test showed functioning p-channel current detector with a $42 \%$ higher threshold than expected $45 \mathrm{~mA}$.

3) Test of gate driver switches:

The gate driver switches need to provide sufficient driving capability for fast switching of the power gates. They were implemented with mid-oxide DMOS technology. Due to similar requirements for both sets of gate driver and cap driver switches, the same nmos/pmos pair was used to implement gate drive and cap drive. Due to easy control of switch states, the onresistance and body diode conduction effect were performed on cap switches.

a. N-channel test of on-resistance and body diode

i. Set gd_ctrl (pin 14) to low, hl_sel (pin 18) to low;

ii. Inject $\pm 100 \mathrm{~mA}$ current from voltage source into cap (pin 5) through a resistor, measure injected current and cap voltage.

Table 5-8. Test results of on-resistance of n-channel cap switch:

\begin{tabular}{|c|c|c|c|c|c|c|}
\hline \multirow{2}{*}{$\begin{array}{c}\text { Injected } \\
\text { Current }(\mathrm{mA})\end{array}$} & \multicolumn{3}{|c|}{ Voltage @ pin 5 } & \multicolumn{3}{c|}{ Calculated On-Resistance $(\Omega)$} \\
\cline { 2 - 7 } & $\# 1$ & $\# 2$ & $\# 3$ & $\# 1$ & $\# 2$ & $\# 3$ \\
\hline 100 & 2.44 & 2.38 & 2.24 & 24.4 & 23.8 & 22.4 \\
\hline 80 & 1.81 & 1.80 & 1.73 & 22.6 & 22.5 & 21.6 \\
\hline 60 & 1.28 & 1.29 & 1.25 & 21.3 & 21.5 & 20.8 \\
\hline 40 & .816 & .82 & 0.79 & 20.4 & 20.5 & 19.8 \\
\hline 20 & .395 & .39 & 0.39 & 19.8 & 19.5 & 19.5 \\
\hline-20 & -.384 & -.36 & -0.37 & 19.2 & 18.0 & 18.5 \\
\hline-40 & -.698 & -.69 & -0.69 & 17.5 & 17.3 & 17.2 \\
\hline-60 & -.783 & -.78 & -0.78 & 13.1 & 13.0 & 13.0 \\
\hline-80 & -.820 & -.82 & -0.81 & 10.3 & 10.3 & 10.1 \\
\hline-100 & -.839 & -.85 & -0.84 & 8.4 & 8.5 & 8.4 \\
\hline
\end{tabular}


Discussion: This test measures the on resistance of the n-channel FET by setting gd_ctrl low and injecting current into the cap pin. The body diode conduction voltage is also found by monitoring the cap voltage $(\sim 0.7 \mathrm{~V})$. The on-resistance of all 3 chips is within $20 \%$ of the expected $18 \mathrm{ohms}$ for currents between -50 and $80 \mathrm{~mA}$, as shown in Figure 5-5.

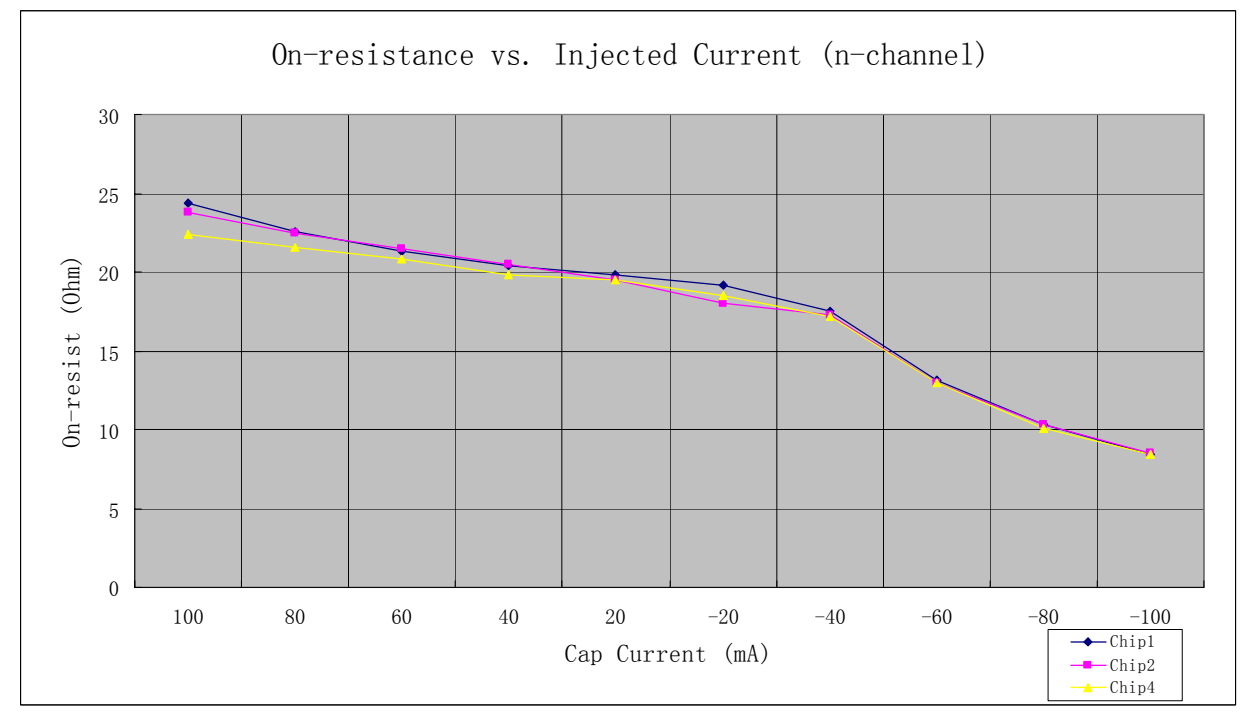

Figure 5-5. On-resistance of n-channel MOSFET

b. P-channel test of on-resistance and body diode

i. $\quad$ Set gd_ctrl (pin 14) to high (5V), hl_sel (pin 18) to low;

ii. Inject $\pm 100 \mathrm{~mA}$ current from voltage source into cap (pin 5) through a resistor, measure injected current and cap voltage.

Table 5-9. Test results of on-resistance of p-channel cap switch:

\begin{tabular}{|c|c|c|c|c|c|c|}
\hline \multirow{2}{*}{$\begin{array}{c}\text { Injected } \\
\text { Current }(\mathrm{mA})\end{array}$} & \multicolumn{3}{|c|}{ Voltage @ pin 5 } & \multicolumn{3}{c|}{ Calculated On-Resistance $(\Omega)$} \\
\cline { 2 - 7 } & $\# 1$ & $\# 2$ & $\# 3$ & $\# 1$ & $\# 2$ & $\# 3$ \\
\hline-100 & 14.7 & 14.87 & 14.81 & 27.0 & 28.70 & 28.1 \\
\hline-80 & 13.5 & 13.55 & 13.55 & 18.8 & 19.38 & 19.4 \\
\hline-60 & 13.2 & 13.25 & 13.28 & 20.0 & 20.83 & 21.3 \\
\hline-40 & 13.1 & 13.18 & 13.20 & 27.5 & 29.50 & 30.0 \\
\hline-20 & 12.8 & 12.80 & 12.79 & 40.0 & 40.00 & 39.5 \\
\hline 20 & 11.3 & 11.33 & 11.33 & 35.0 & 33.50 & 33.5 \\
\hline 40 & 10.7 & 10.72 & 10.69 & 32.5 & 32.00 & 32.8 \\
\hline 60 & 9.97 & 10.05 & 9.99 & 33.8 & 32.50 & 33.5 \\
\hline 84 & 8.90 & 9.01 & 8.99 & 36.9 & 35.60 & 35.8 \\
\hline 100 & 8.04 & 8.08 & 8.41 & 39.6 & 39.20 & 35.9 \\
\hline
\end{tabular}


Discussion: This test is the same as above, except the p-channel FET is now tested. The on resistance is within $20 \%$ of the expected $25 \mathrm{Ohms}$ for Cap currents between -100 and $-40 \mathrm{~mA}$, as shown in F. Body diode conduction voltage is around $13 \mathrm{~V}$.

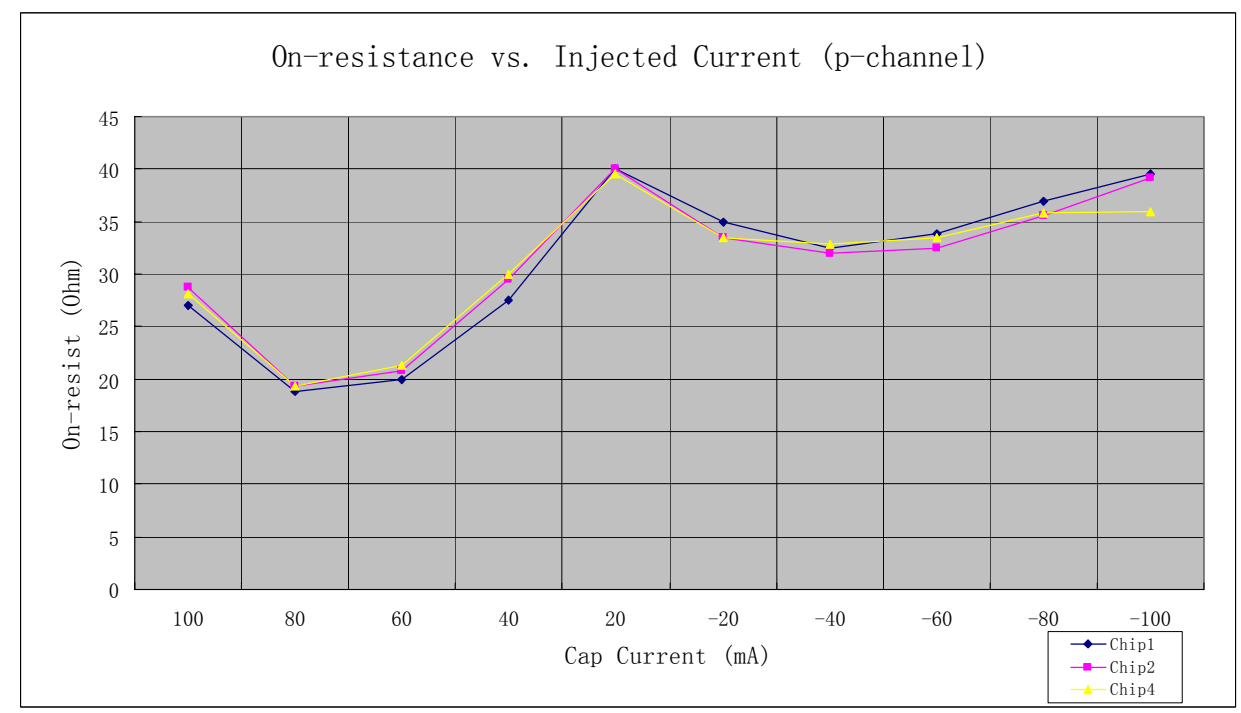

Figure 5-6. On-resistance of p-channel MOSFET

\subsubsection{Digital Logic Test (LS and HS Communication):}

The control logic performs the state machine (HS and LS) operation based on the control input (gd_ctrl) and outputs of current detectors (signaling and handshaking detectors) to generate proper signal sequencing to the cap and gate switches to drive the coupling capacitor and the power switch gates. Proper operation of the digital control logic could be verified by examining the waveforms of LS and HS cap and gate switches. Test procedure is:

i. Configure half-bridge with LSIC, HSIC and $320 \mathrm{pF}$ coupling capactor;

ii. Short the half-bridge midpoint voltage to ground temporarily;

iii. Apply LS and HS power supplies directly to the two IC power supplies;

iv. Apply the control signal (gd_ctrl) to LSIC pin 14, record cap and gate wavefroms.

Test results: Figure 5-7 shows measured LS and HS gate and cap output waveforms under difference combination of LS and HS supply voltage. It can be seen that under nominal $12 \mathrm{~V}$ supply voltage there was no HS gate signal due to wrong cap switching. When increasing HS voltage to be $14 \mathrm{~V}$, correct HS gate and cap switching was obtained. However, more LS gate signals were missing with increased LS supply voltage. As shown in Figure 5-7 the occurrence of expected LS gate signal increases with the decrease of LS supply voltage. To get correct signaling and handshaking detection, LS and HS supply voltages are swept through entire design 
range. With $320 \mathrm{pF}$ coupling capacitance, the operation area was obtained, as shown in Figure 5-8. The lower boundary of HS supply voltage was found to be $14 \mathrm{~V}$, below which HSIC cannot detect signaling current. The upper boundary is limited by the CMOS process, which is $20 \mathrm{~V}$. The upper boundary of LS supply voltage was found to be $11.8 \mathrm{~V}$, above which LSIC tends to miss handshaking detection. The lower boundary is set by on-chip 5V linear voltage regulator, below which the digital circuitry cannot work.
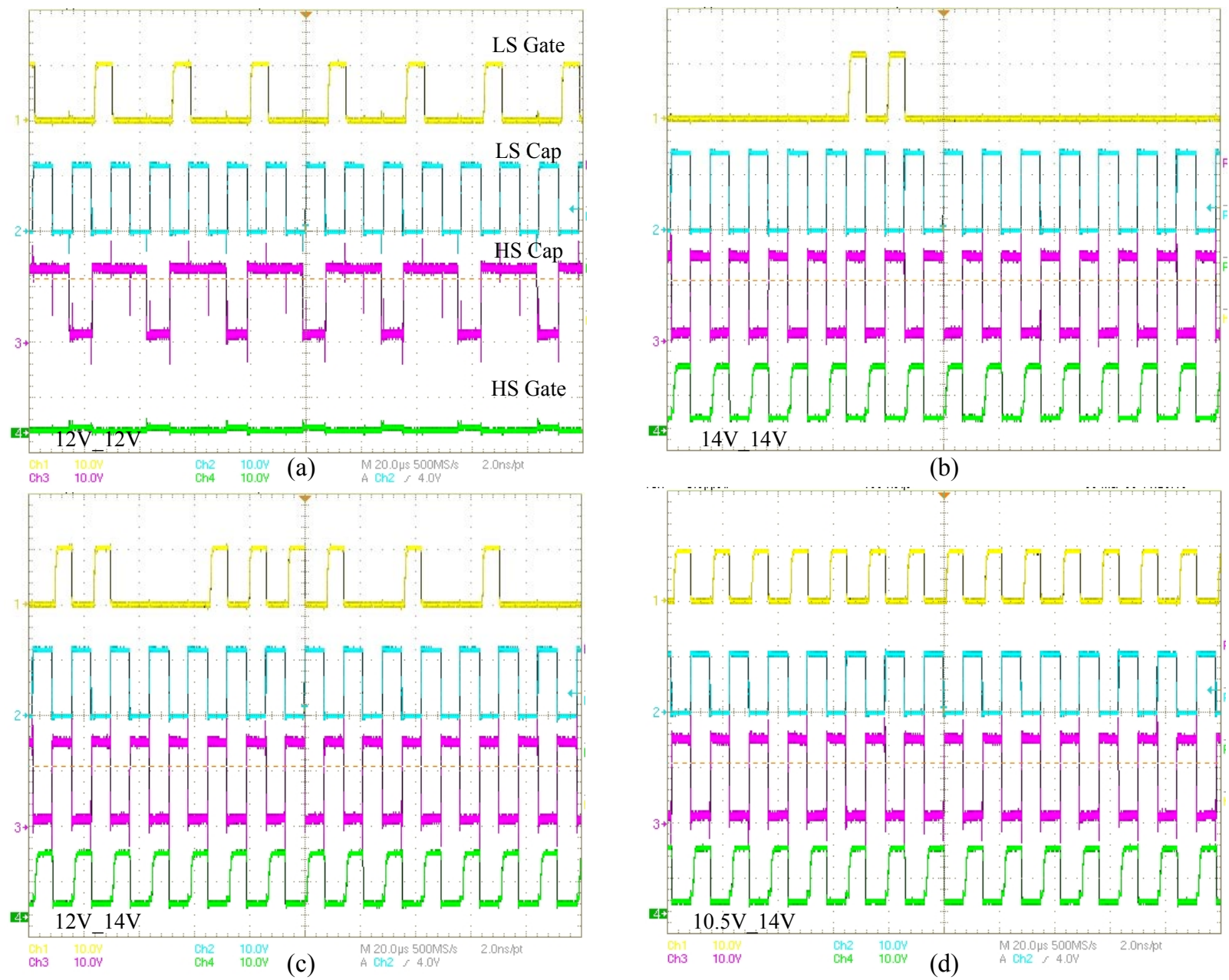

Figure 5-7. Experimental results showing correct logic control operation depending on correct detection of signaling and handshaking currents; a) HSIC failed to detect rising transition; b) Unreliable handshaking detection of LSIC; c) Occurrence of correct handshaking detection of LSIC increases with decrease of supply voltage; d) Correct operation with $10.5 \mathrm{~V}$ LS supply voltage and $14 \mathrm{~V}$ HS supply voltage; ch1: LS gate drive; ch2: LS cap drive; ch3: HS cap drive; ch4: HS gate drive; All with 10V/div. 


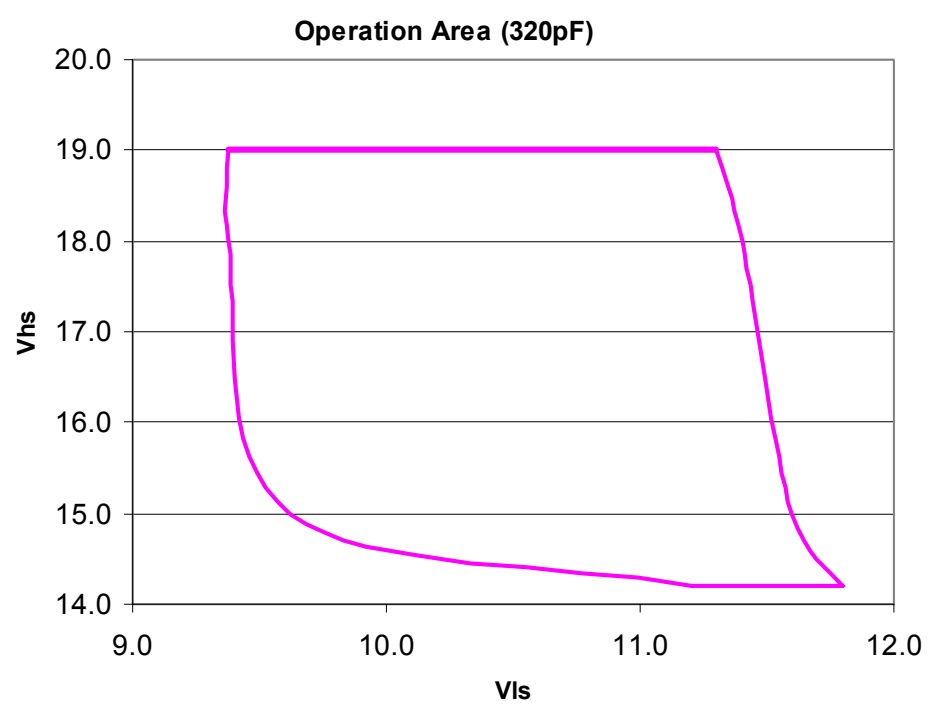

Figure 5-8. Operation area of LS and HS supply voltage, inside purple area LS and HS ICs can communicate each other

Discussion: The ICs were designed for nominal $12 \mathrm{~V}$ supplies. However, the bias performance test (bias block test (a) and (c)) showed that on-chip current and voltage references were about $50 \%$ and $20 \%$ higher than respective nominal values due to an out-of-spec shift in process parameters. This out-of-spec shift was also verified by analog performance test a) to c). The consequences of this shift affect the detection of signaling current (HSIC) and handshaking current (LSIC), as shown in Figure 5-7(a). It is reasonable to think that the IC supply voltage should increase at least $20 \%$, or $14.4 \mathrm{~V}$, accordingly. However, test results shown in Figure 5-7 and Figure 5-8 only agree with this assumption for HSIC. For LSIC, the supply voltage should be lower than $12 \mathrm{~V}$ in order to get correct operation. What happens when LS supply voltage excesses the upper boundary shown in Figure 5-8? To answer this question, the waveforms of control signal gd_ctrl, LS gate drive, LS cap drive, and HS cap drive signals were captured, as shown in Figure 5-9. It can be seen that under 14V LS supply voltage, some LS gate drive signals missed. The detailed waveforms are expanded at three different locations showing a correct falling transition (Figure 5-9a), correct rising transition (Figure 5-9b), and wrong falling transition (intended) (Figure 5-9c). During a correct falling transition, LS cap switch switches from Vdd to Vss with the falling edge of control signal, gd_ctrl. The switching of LS cap switch causes signaling current to HSIC (a little dip shown in HS cap waveform). Following detection of the signaling current, HS cap switches from Vss to Vdd, causing handshaking current pulse to LSIC (a little bump shown in LS cap waveform). HSIC then turns gate off, initialing a falling transition. After a deadtime, the LSIC turns the gate on. For a correct falling transition a similar process occurs. During a wrong falling transition, the detection of signaling current is correct, but the HSIC sends a handshaking current pulse to LSIC prematurely with a separation time around $40 \mathrm{~ns}$, well below normal 100ns. Since the width of the signaling and handshaking current pulse is about 50 100ns (as shown in Figure 3-8), two pulses would be superimposed, resulting 
in a failure detection of handshaking current in LSIC. Therefore, the LS gate keeps low, as commanded by the state machine in Figure 3-14(a). However, the question remains as to why LS supply voltage affects the HS signaling detection. One possible explanation is that the LS voltage affects the magnitude and shape of the signaling current pulse. However, by setting a lower threshold current in HS (15mA in HS v.s. $45 \mathrm{~mA}$ in LS) would ensure the first falling edge of vdet_n in LS occurs before the first rising edge of vdet_n in HS so that HS would send out handshaking current after first current pulse disappeared.
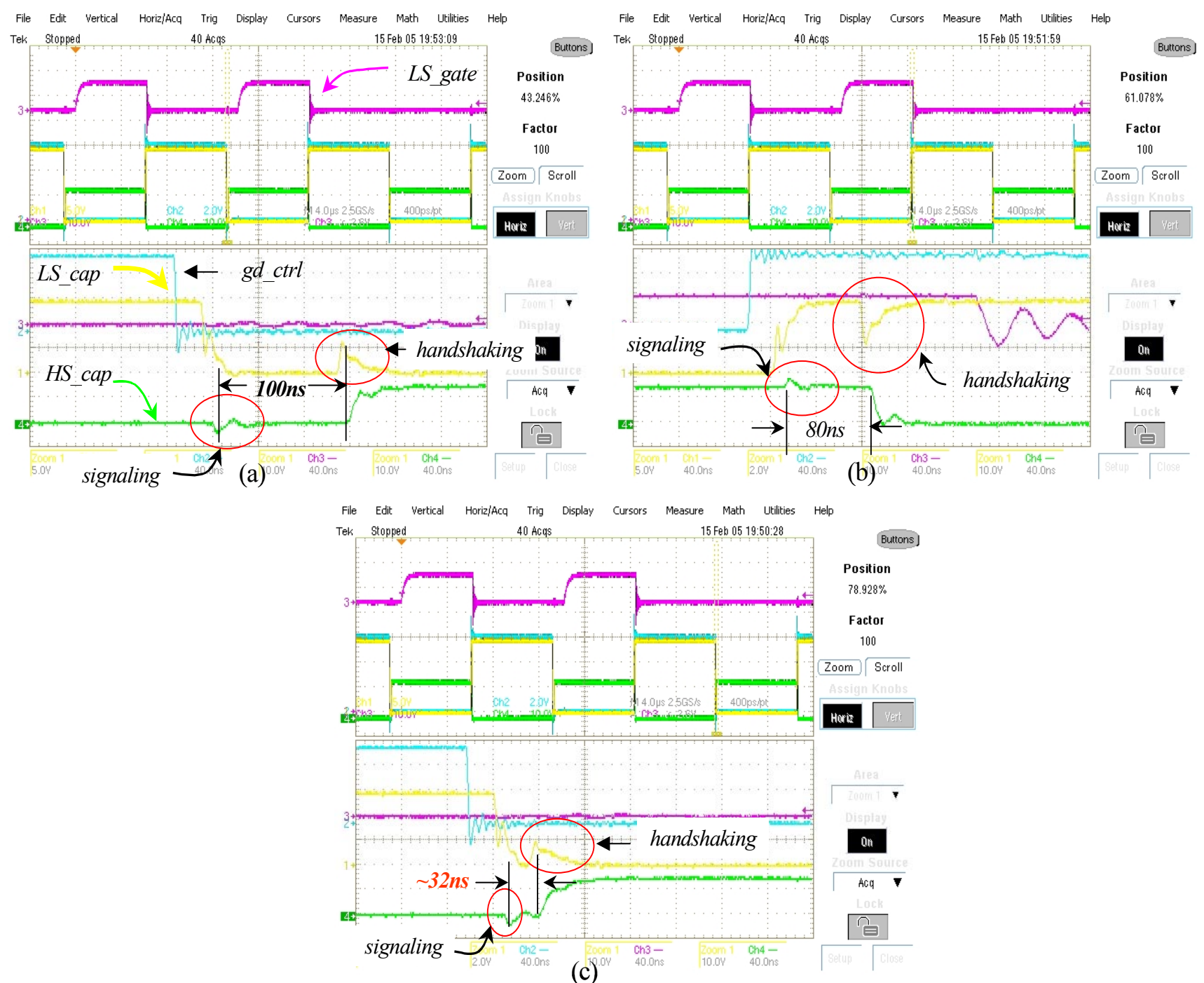

Figure 5-9. Detailed waveforms showing communication between LS and HS ICs with 14 V LS and HS supply voltage: a) a proper falling transition, b) a proper rising transition, c) missed LS gate drive in falling transition 


\subsubsection{High Voltage Test}

After setting proper LS and HS supply voltage, a high voltage test was performed to demonstrate signaling and handshaking behavior between the two ICs by drive a $200 \mathrm{~V}$ half-bridge circuit, as shown in Figure 5-10. Figure 5-11 shows the measured voltage waveforms on the two sides of the coupling capacitor for the rising transition under ZVS conditions (reference Figure 3-2(b) and Figure 3-3). The pulses in these voltages are proportional to the current pulses in the coupling capacitor as the current flows through the on-resistance of the on-chip devices. First, the LSIC switch S1 goes high, which positions the LSIC charge pump and signals to the HSIC. Following detection of the current pulse, the HSIC switch S3 goes low, positing the HSIC charge pump and providing a handshaking pulse to the LSIC. The LSIC gate switch S2 then goes low, initiating a rise in the mid-point voltage Vm (due to ZVS). After a deadtime, the HSIC gate switch S4 goes high (not shown on this time scale). As shown, two ICs operated with continuous successful communication during high voltage applications.

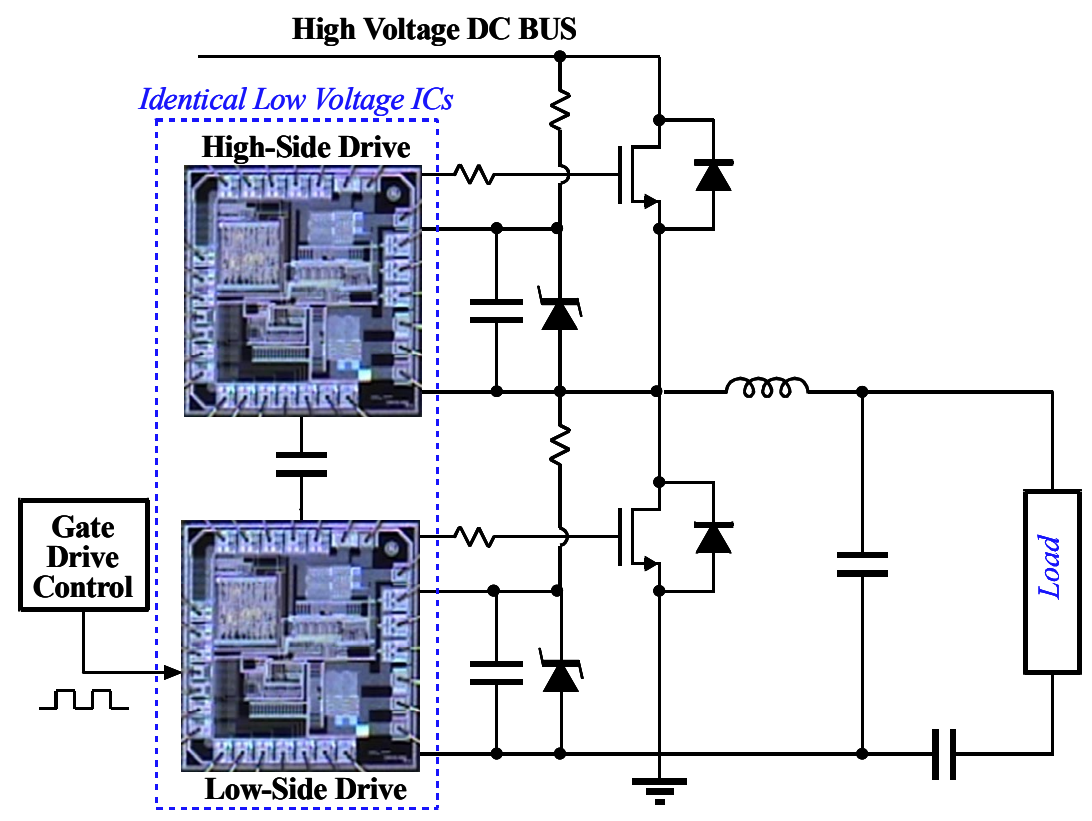

Figure 5-10. High Voltage Test Setup for demonstration of dual LVIC operation 


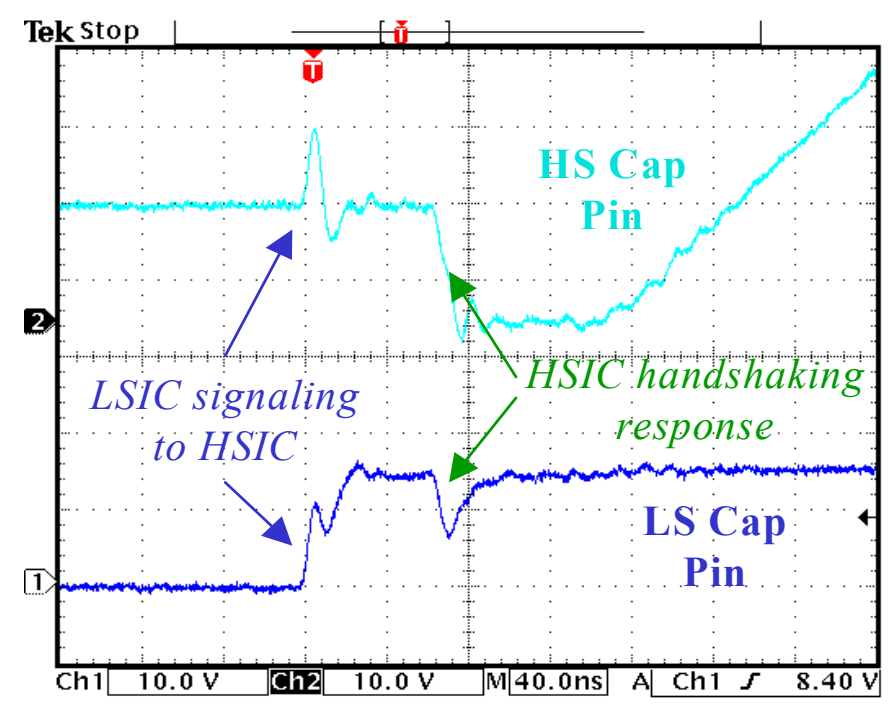

Figure 5-11. Experimental results showing the two sides of the coupling capacitor pin (LS and HS) during signaling associated with the rising transition

\subsection{Ballast Controller IC Test:}

The tests were performed to verify proper operation of the analog interface and digital control functions. The analog interface testing includes inductor current zero crossing detection and lamp current/voltage rectification and $\mathrm{A} / \mathrm{D}$ conversion. The digital control functions include closed-loop regulation and start-up behavior.

\subsubsection{Auxiliary Circuit Test (Bias and Clock Generation Blocks)}

1) On-chip current bias test

a) Measure the resistance Ri from TP2 to gnd.

b) Apply $5 \mathrm{~V}$ and measure the voltage Vi of TP2;

c) Adjust pot R25 (refer to schematic) to make Vi/Ri close $25 \mathrm{uA}$.

Table 5-10. Test results of current bias:

\begin{tabular}{|c|c|c|c|}
\hline & $\# 1$ & $\# 2$ & $\# 3$ \\
\hline $\mathrm{R}_{\mathrm{i}}(\mathrm{k} \Omega)$ & 137 & 138 & 136 \\
\hline $\mathrm{V}_{\mathrm{i}}(\mathrm{V})$ & 3.42 & 3.45 & 3.41 \\
\hline
\end{tabular}

Discussion: This test verifies the bias block's ability to generate desired current bias. The spread among chips was small, within a few percent of each other.

2) On-chip $100 \mathrm{MHz}$ test

a) Set test $=0$ (use the jumper to connect test to gnd); 
b) Set st_per_sel = 2'b11;

c) Apply 5V;

d) Measure the frequency of the gate drive output (gate_l or gate_h);

e) Adjust pot R24 to make the gate drive frequency is close to $250 \mathrm{kHz}$;

f) Change the setting of st_per_sel to 00,01, and 10 and measure the gate drive frequency;

g) Record the deadtime between gate_l and gate_h.

Table 5-11. Test result of on-chip 100MHz block

\begin{tabular}{|c|c|c|c|c|}
\hline st_per_sel & 00 & 01 & 10 & 11 \\
\hline frequency & $72 \mathrm{kHz}$ & $100 \mathrm{kHz}$ & $167 \mathrm{kHz}$ & $250 \mathrm{kHz}$ \\
\hline \multicolumn{5}{|c|}{} \\
\hline dt_sel & 00 & 01 & 10 & 11 \\
\hline deadtime & $600 \mathrm{~ns}$ & $450 \mathrm{~ns}$ & $300 \mathrm{~ns}$ & $1.2 \mu \mathrm{s}$ \\
\hline
\end{tabular}

Discussion: This test verifies the clock block's ability to generate different DCO frequencies at each of the st_per_sel settings and different dead times at each of the dt_sel settings.

3) Internal $\mathrm{A} / \mathrm{D}$ clock test

a) Set clk_adc_sel =0 (use internal clock);

b) Input a sinusoidal voltage in i_ind_p and i_ind_m;

c) Monitor the output of clk_adc_out (short the jumper).

Discussion: The frequency of the clock output is 32 times (adc_sel $=0$ ) or 16 times (adc_sel $=1$ ) of that of the input signal with negative duty cycle is $25 \%$, as shown in Figure 5-12.

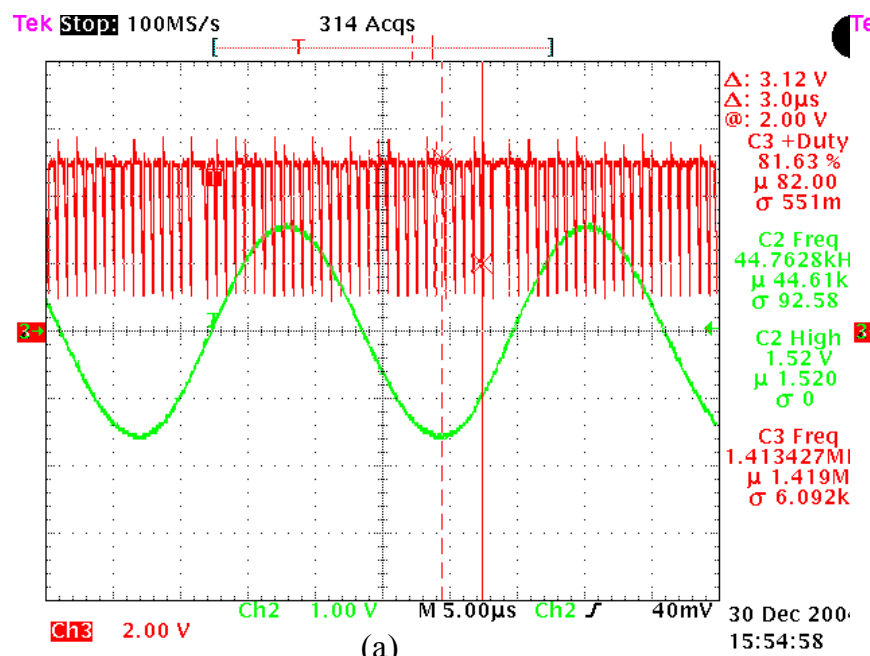

(a)

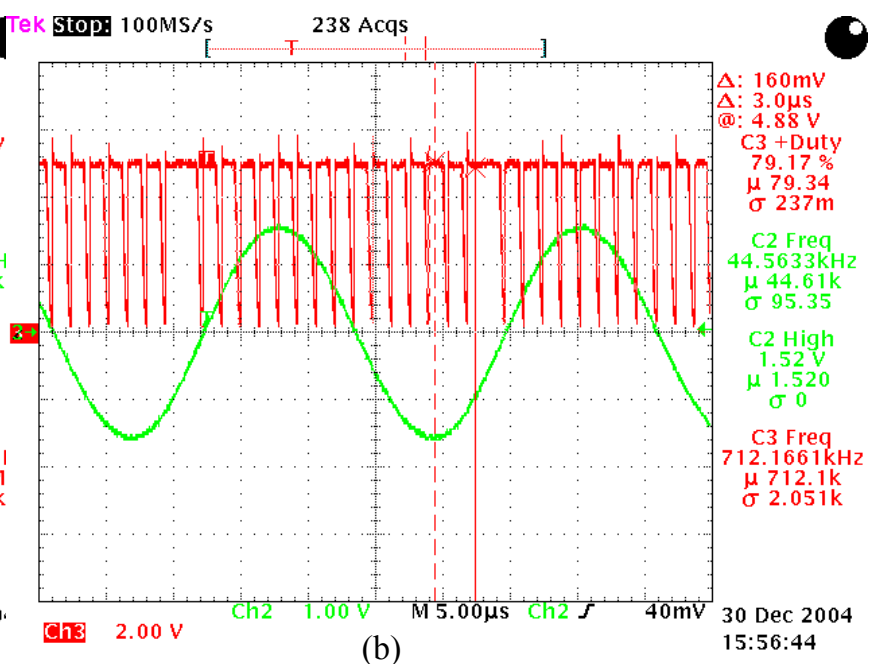

(b)

Figure 5-12. Internal $A / D$ test with $44 \mathrm{kHz}$ input showing a correct clock frequency generated based on adc_sel settings; (a) $32 \mathrm{x}$ fs with adc_sel $=0$; (b) $16 \mathrm{x}$ fs with adc_sel $=1$ 


\subsubsection{Analog Interface}

1) $\mathrm{AC}$ buffer and inductor current zero crossing detection:

The ac buffer converts the ac voltage to an ac current that can be processed by the following current mode comparator or current-mode A/D converter. The current-mode design can get around the requirement to generate the negative power supply for the voltage-mode design in a standalone system. However, some dc-offset current introduced by ac buffer were noticed during the test. This problem can be overcome by a tighter process or injecting current into the ac buffer to compensate for the offset. Please refer to appendix for the detailed cancellation process. As shown in Figure 5-13 a $2.016 \mathrm{M} \Omega$ bias resistor $(4 \mathrm{~V} / 2 \mu \mathrm{A})$ was used to inject current into negative input. It can be seen that duty ratio keeps 0.5 over a wide range of input magnitude and frequency.
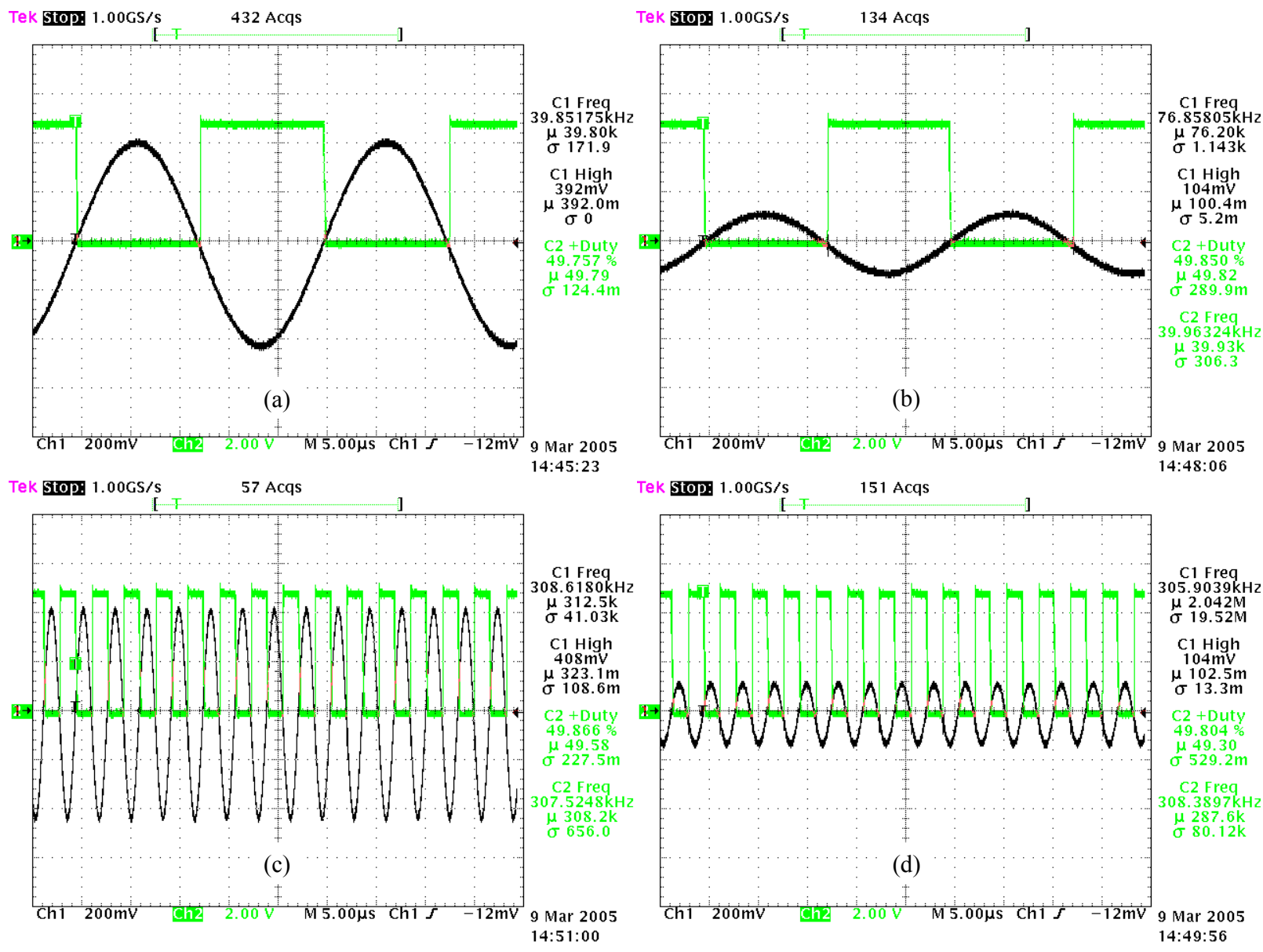

Figure 5-13. Zero crossing detection results demonstrating effect of dc-injection cancellation: duty ratio keeps 0.5 over wide range of input magnitude and frequency: (a) $V_{\text {in }}=0.4 \mathrm{~V}$, $40 \mathrm{kHz}$; (b) $V_{\text {in }}=0.1 \mathrm{~V}, 40 \mathrm{kHz}$; (c) $V_{\text {in }}=0.4 \mathrm{~V}, 300 \mathrm{kHz}$; (d) $V_{\text {in }}=0.1 \mathrm{~V}, 300 \mathrm{kHz}$ 
The magnitude of dc-bias current in current/voltage channel could be estimated from the current difference between two peaks of rectifier waveform, as illustrated in Figure 5-14. For the case shown in Figure 5-14, a 2.228M $\Omega$ resistor was used to inject current into positive input and waveform becomes symmetrical, as shown in Figure 5-14(b).

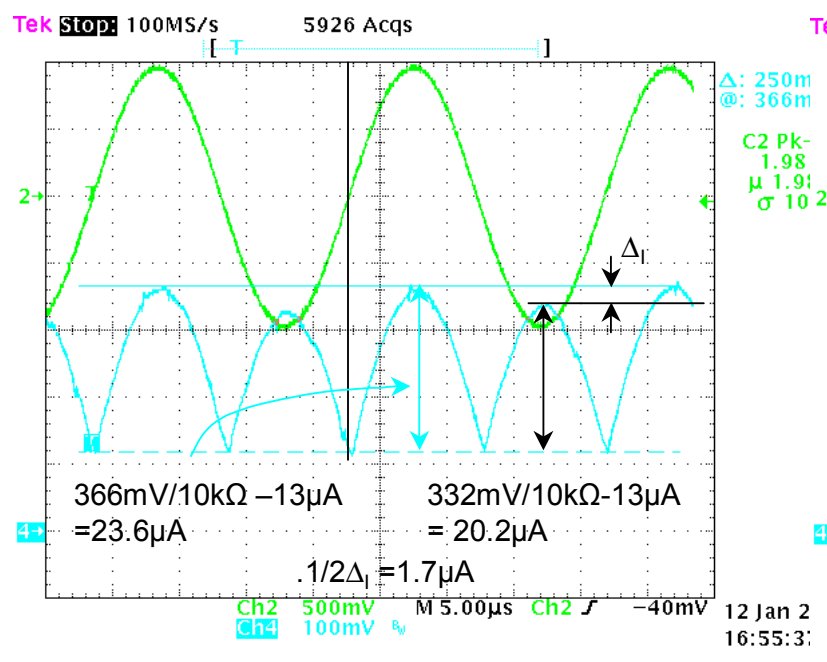

(a)

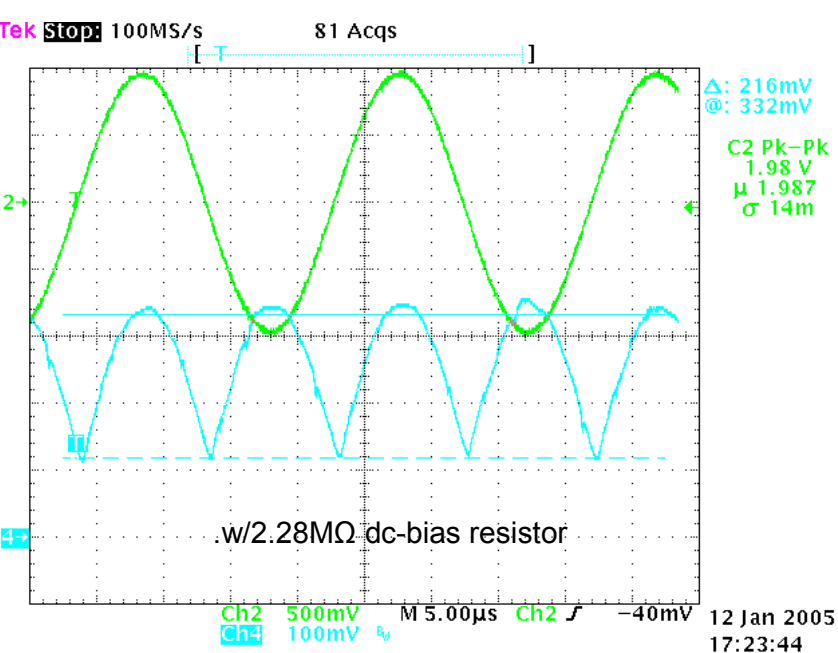

(b)

Figure 5-14. Output current waveforms from I-mode rectifier showing a) dc-offset caused asymmetric peaks, b) symmetric peaks with $\sim 1.7 \mu \mathrm{A}$ injected into positive input

\section{2) Current-mode A/D Conversion:}

The lamp current channel was used to test the rectification and A/D conversion circuitry. The external matching and dc-bias resistors were: $\mathrm{R}_{\mathrm{m}}=39.2 \mathrm{k} \Omega, \mathrm{R}_{\mathrm{b}}=161.2 \mathrm{k} \Omega$ and $\mathrm{R}_{\mathrm{dc} \text { bias }}=3.36 \mathrm{M} \Omega$ (to cancel out $1.19 \mu \mathrm{A}$ dc offset current). Use the logic analyzer to watch the digital outputs of i_lamp_[0:5] by setting the logic analyzer as: a) use clk_adc_out as the logic analyzer clock; b) in the format menu, set the clock as "user" and trigger level as $3.5 \mathrm{~V}$; c) in the configuration menu, set type as "state"; d) watch the digital outputs in "chart machine". The outputs should be full-wave rectifier sinusoidal wave with constant peak value, as shown in Figure 5-15, which illustrates the dc-offset cancellation effect with and without the dc-bias resistor connected.

As shown in Figure 5-16 an interesting phenomenon was observed when the linearity of the A/D conversion circuitry was tested. The digital output remains constant as the input voltage reaches some value $(0.58 \mathrm{~V}$ peak in shown case). This observation prompted more detailed testing of the pipelined current mode A/D converter with respect to accuracy and stability tests. Accuracy is measured in terms of the average digital output for a given analog input since the digital output often oscillates \pm 1 LSB. Stability is measured in terms of the number of digital values, which spike outside a specified range about the average digital output. Results were obtained using the Harrison Lab DC power supply to provide the analog voltage to $v_{i n}$ of the lamp current AC buffer the HP logic analyzer to record the digital output. The input voltage was progressively adjusted to achieve the full digital output range in increments of one LSB. 
In order to verify that any conversion glitches were not caused by sample rate limitations, it was desired to test the A/D converter at a slow sample rates as well as at the normal sampling rates of $16 x$ and $32 x$ the switching frequency. With the chip operating in DCO mode with a $6 \mu$ s period, the normal sampling rates would be $2.7 \mathrm{MHz}$ and $5.3 \mathrm{MHz}$, respectively. Slower clocks could be achieved by setting the $c l k$ adc_sel switch to external ADC clock and using the external clock input clk_adc_in. A sample rate of $1 \mathrm{x}$ the switching frequency, or $167 \mathrm{kHz}$, was achieved by using gate_s gate drive output to supply the external clock. The function generator was used to supply an even slower clock of $10 \mathrm{kHz}$. It should be noted that the duty cycle of the internal A/D clock is $25 \%$, not $50 \%$. Therefore the sample and hold circuitry is active for the first $25 \%$ of the sample period, and the pipelined A/D converter is active for the remaining $75 \%$ of the sample period and the $\mathrm{A} / \mathrm{D}$ conversion time does not scale linearly when going from an internal to external (50\% duty cycle) clock. The clock frequency, sample and hold time, and A/D conversion time for each AD clock used are listed in Table 5-1.

Table 5-12. Frequency and conversion time test of $A / D$ converter

\begin{tabular}{|c|c|c|c|}
\hline Clock Frequency & Internal/External & Sample/Hold Time & A/D Conversion Time \\
\hline $10 \mathrm{kHz}$ & External & $50 \mu \mathrm{s}$ & $50 \mu \mathrm{s}$ \\
\hline $167 \mathrm{kHz}$ & External & $3 \mu \mathrm{s}$ & $3 \mu \mathrm{s}$ \\
\hline $2.7 \mathrm{MHz}$ & Internal & $90 \mathrm{~ns}$ & $280 \mathrm{~ns}$ \\
\hline
\end{tabular}




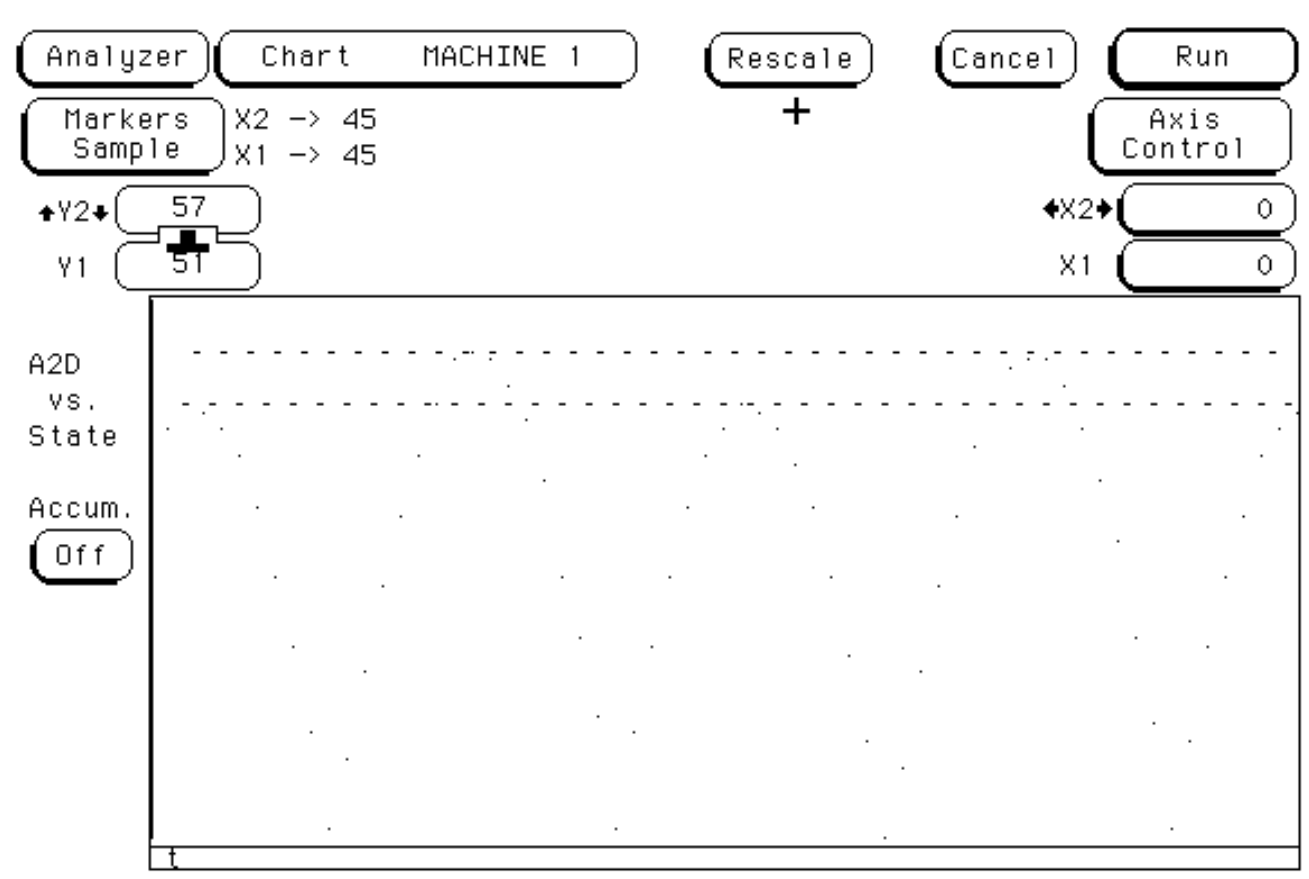

(a)

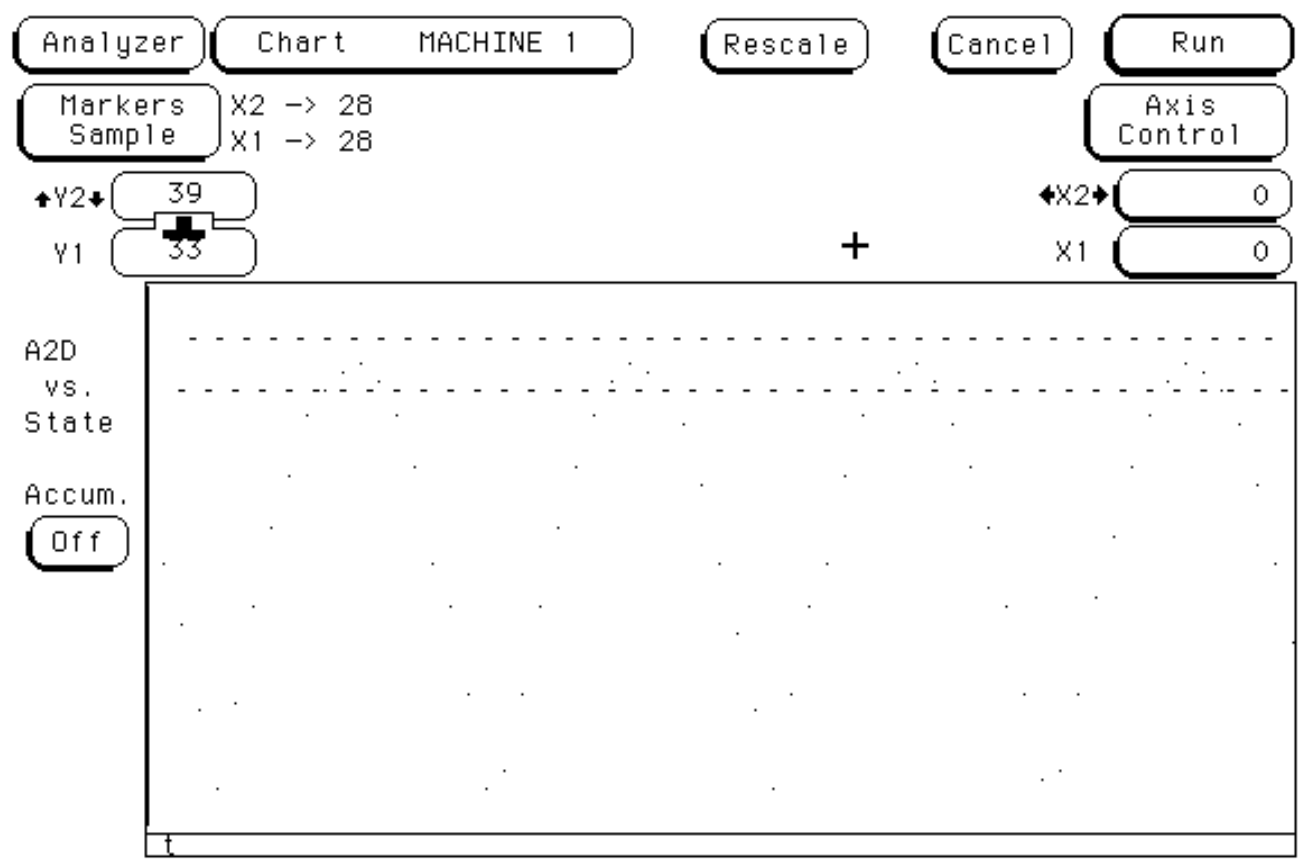

(b)

Figure 5-15. Plots from logic analyzer illustrating the effect of dc-offset cancellation, a) without dcbias resistor connected, b) with resistor connected (the waveforms were inverted) 


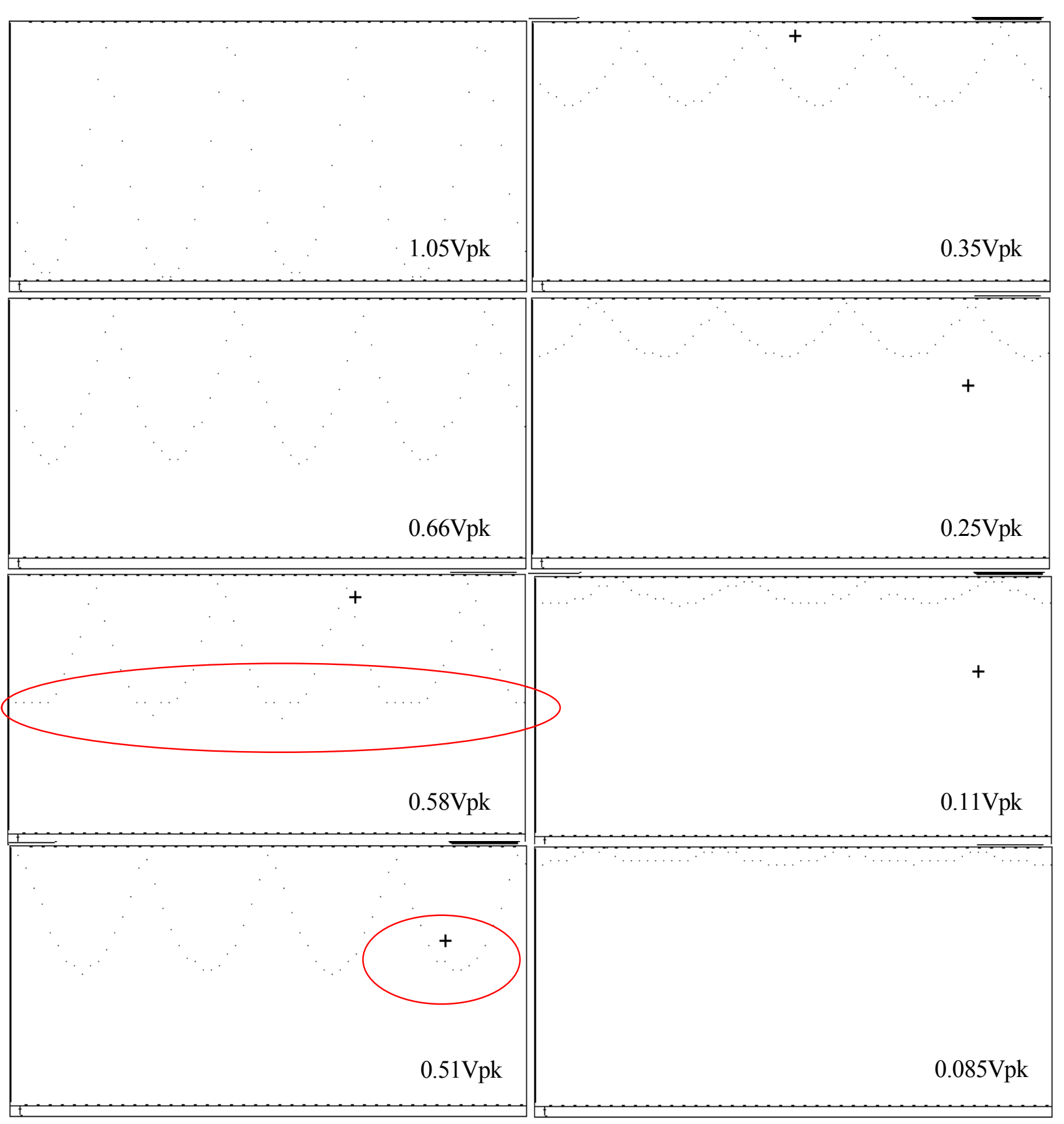

Figure 5-16. Plots from logic analyzer showing the linearity between the input peak voltage and output peak current of $A / D$ circuitry, a "flat spot" was noticed around $55 \%$ of full scale input peak voltage $(0.58 \mathrm{Vpk} / 1.05 \mathrm{Vpk})$

Accuracy test:

Figure 5-17 to Figure 5-19 show plots of average digital output v.s. analog input at the A/D clocks of $10 \mathrm{kHz}, 167 \mathrm{kHz}$, and $2.7 \mathrm{MHz}$ respectively. In these plots, the pink squares and left- 
hand side y-axis is the raw digital value in decimal. The black diamonds and right-hand side yaxis is the digital value scaled by full scale of $10 \mathrm{~A}$ for the 6 -bit converter $(10 \mathrm{~A}=3 \mathrm{FH}$ or $63 \mathrm{~d})$. The linear trend line is fit to the scaled digital output. Data for these plots was collected after current injection had been added to remove (as much as possible) the DC offset introduced by the AC buffer.

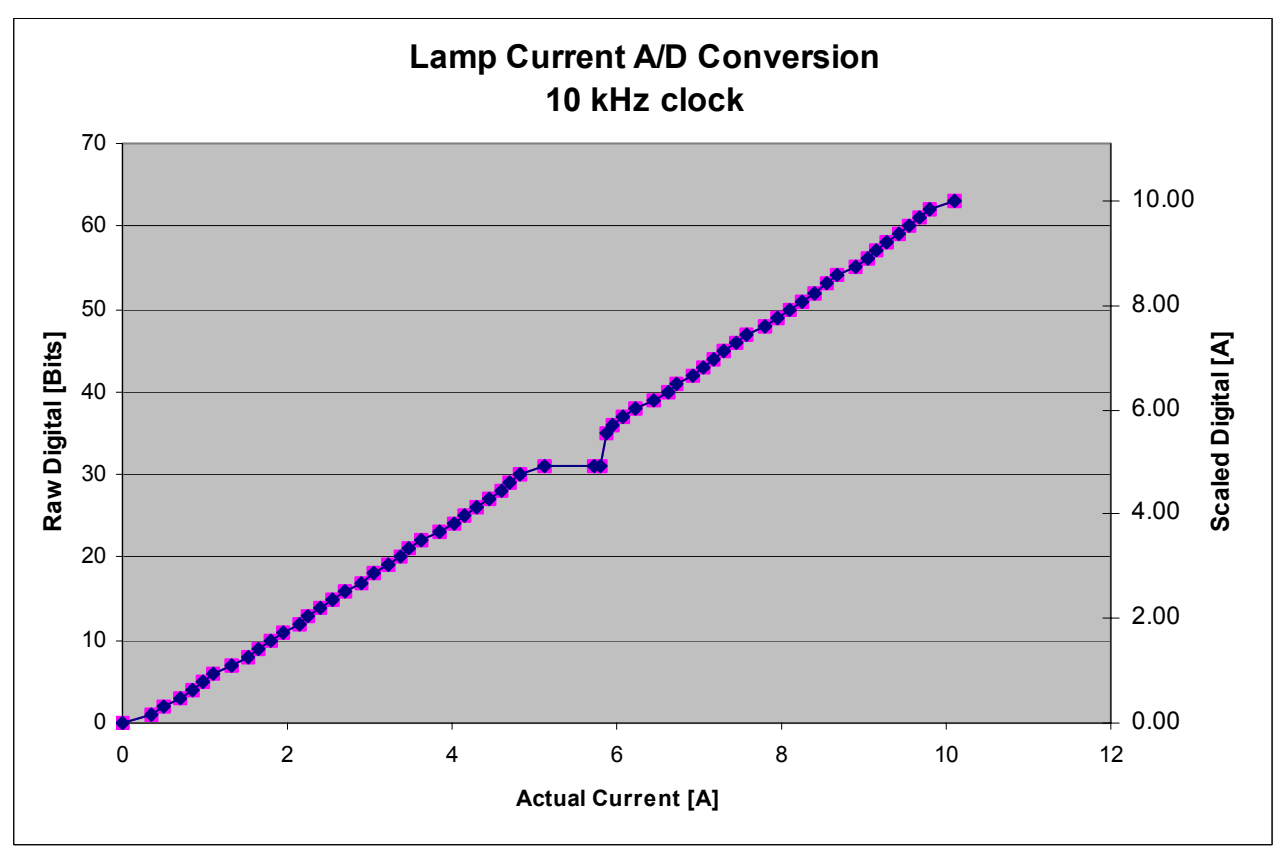

Figure 5-17. Average digital output v.s. actual current (emulated by DC power supply) with an external $\mathrm{A} / \mathrm{D}$ clock at $10 \mathrm{kHz}$ 


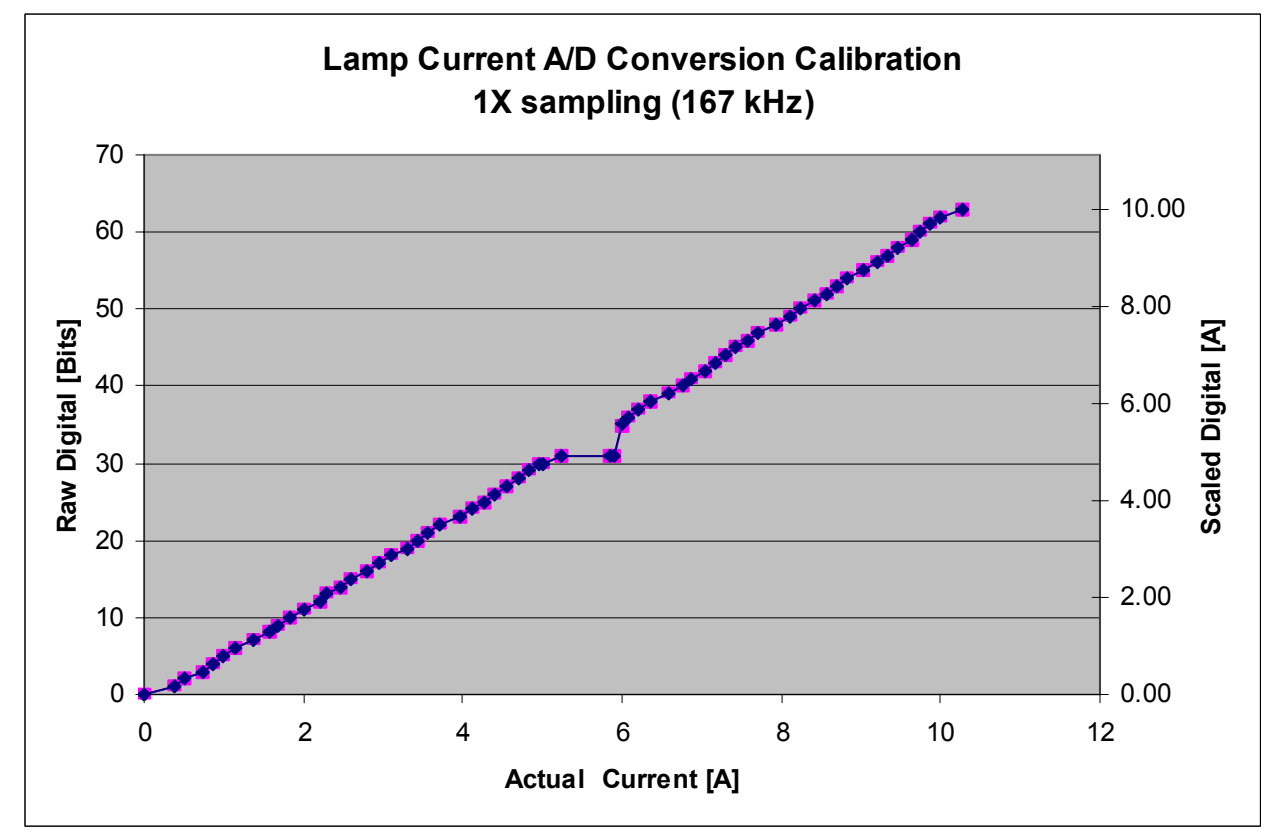

Figure 5-18. Average digital output vs. actual current with an external A/D clock at $167 \mathrm{kHz}$

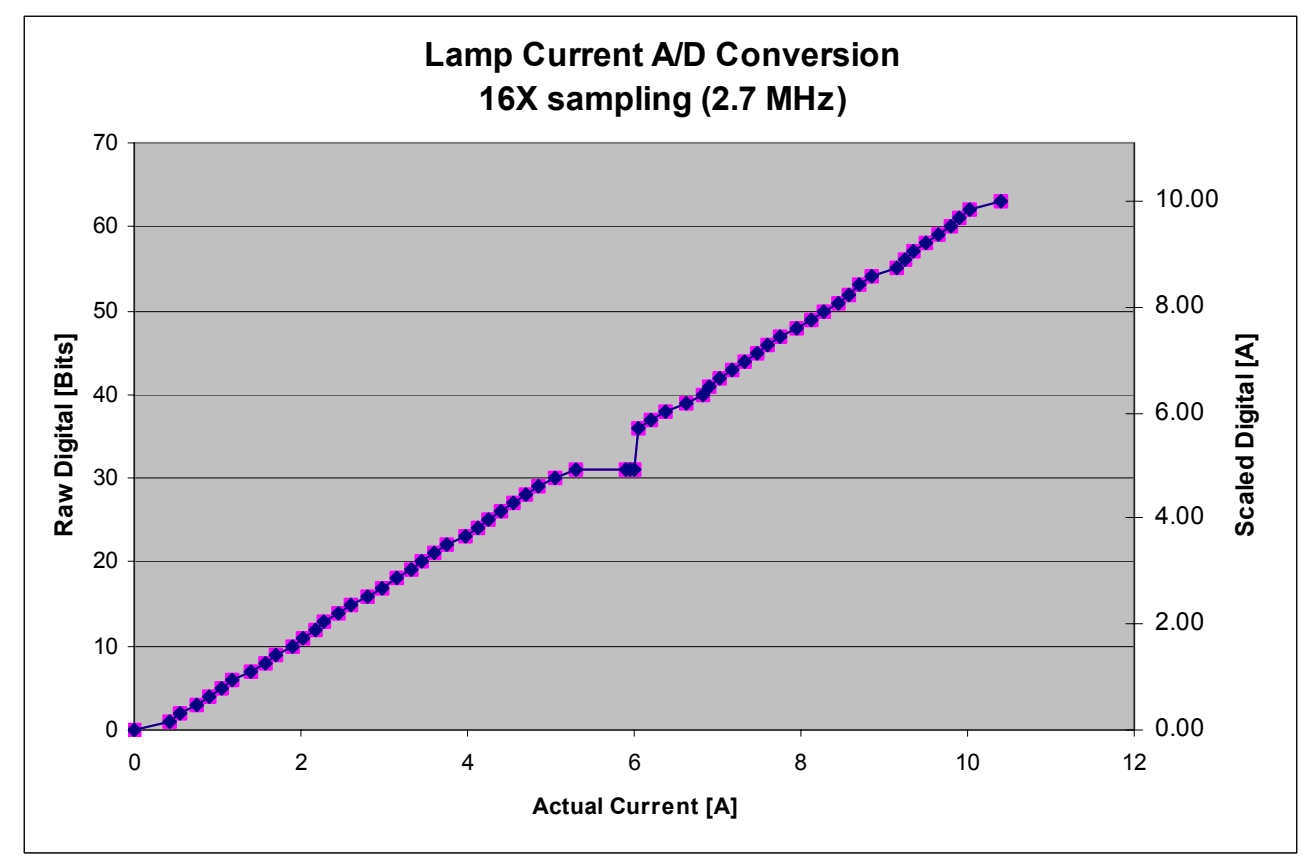

Figure 5-19. Average digital output vs. actual current with internal A/D clock at $2.7 \mathrm{MHz}$ and $300 \mathrm{~W}$ output power

It should be noted that the transitions from one digital value to the next are not abrupt. Rather, from any given digital output, as the analog voltage is increased, the digital output will begin to 
jump with increasing frequency to the new value. In some cases, this process will increase until the output is solid at the new value, with very few oscillations up or down. The analog input corresponding to this situation is the one plotted for that digital output. However, in other cases, it is not possible to achieve a solid digital output. Instead, even as the digital output is still jumping from the new output back to the old output, it begins to jump to the next digital output. In these cases, the analog input, which achieves minimal oscillations up and down, is the one plotted for that digital output. Also in this case, the plotted digital output is actually only the average digital output since the output is oscillating \pm 1 LSB.

Figure 5-17 to Figure 5-19 also show that, for all AD sample rates tested, the averaged output is fairly accurate except for the obvious digital glitch at the raw digital value of $31 \mathrm{~d}=1 \mathrm{Fh}(\mathrm{d}$ : decimal, h: hex). When the analog input voltage reaches the value sufficient to produce a $1 \mathrm{Fh}$ digital output, the digital output will remain at $1 \mathrm{Fh}$ even as the input voltage is increased beyond the value at which the digital output should have transitioned to $20 \mathrm{~h}$. In fact, the digital output will remain solid at $1 \mathrm{Fh}$ until the analog input reaches the value sufficient to produce a $22 \mathrm{~h}$ or $23 \mathrm{~h}$ digital output, at which point the digital output will jump from $1 \mathrm{Fh}$ to $22 \mathrm{~h}$ or $23 \mathrm{~h}$ (actually, as with all digital transitions, it will first begin to spike to the new digital value). This same step in values is observed if the analog input is slowly decreased from the equivalent $23 \mathrm{~h}$ value.

Since the same digital glitch is observed even at the slowest AD clock of $10 \mathrm{kHz}$, this is not a sample rate limitation. Furthermore, two additional chips were tested as well as the lamp voltage A/D conversion on the same chip. All chips/channels tested displayed the same problem to varying degrees. One chip lamp current channel skipped all X0h values, e.g. it jumped from $0 \mathrm{Fh}$ to $11 \mathrm{~h}$, from $1 \mathrm{Fh}$ to $22 \mathrm{~h}$, and from $2 \mathrm{Fh}$ to $31 \mathrm{~h}$. But even in this case, the jump at $1 \mathrm{Fh}$ was the largest. It appears there is a problem rolling over from $1 \mathrm{Fh}=011111 \mathrm{~b}$ to $20 \mathrm{~h}=100000 \mathrm{~b}$, or more generally perhaps, a problem in rolling over the four LSBs from 1111 to 0000 . This problem may be related to the stability problems described next.

\section{Stability test:}

Figure 5-20 to Figure 5-22 show the number of digital spikes outside a \pm 2 bit window for each average digital output at the A/D clocks of $10 \mathrm{kHz}, 167 \mathrm{kHz}$, and $2.7 \mathrm{MHz}$ respectively. In these plots, the average digital output is displayed along the $\mathrm{x}$-axis (in decimal) and the number of spikes outside the specified window is displayed along the $y$-axis as a percentage of the 4000 data points collected at each average digital output. For the $10 \mathrm{kHz}$ and $167 \mathrm{kHz}$ plots, all the non-zero data points are labeled with the average digital value in HEX. Since the $2.7 \mathrm{MHz}$ plot has many data points in the low percentages, only those data points exceeding $0.1 \%$ are labeled. 


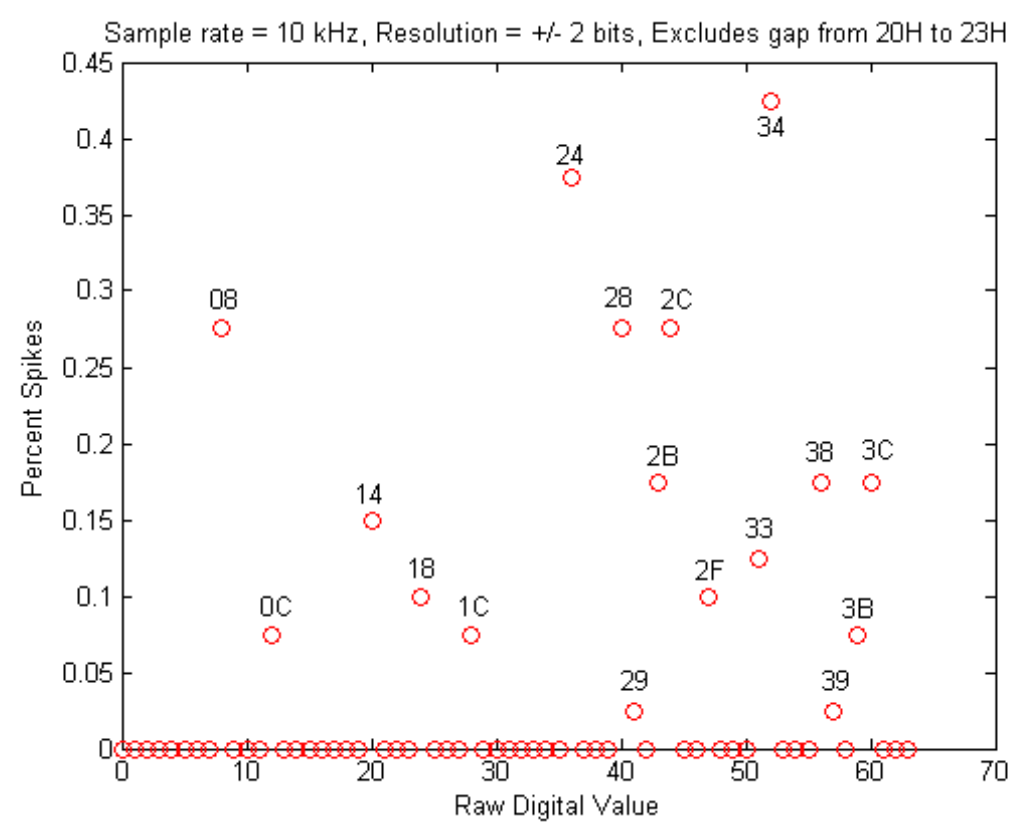

Figure 5-20. Number of digital spikes outside \pm 2-bit window w/ an external $\mathrm{A} / \mathrm{D}$ clock at $10 \mathrm{kHz}$

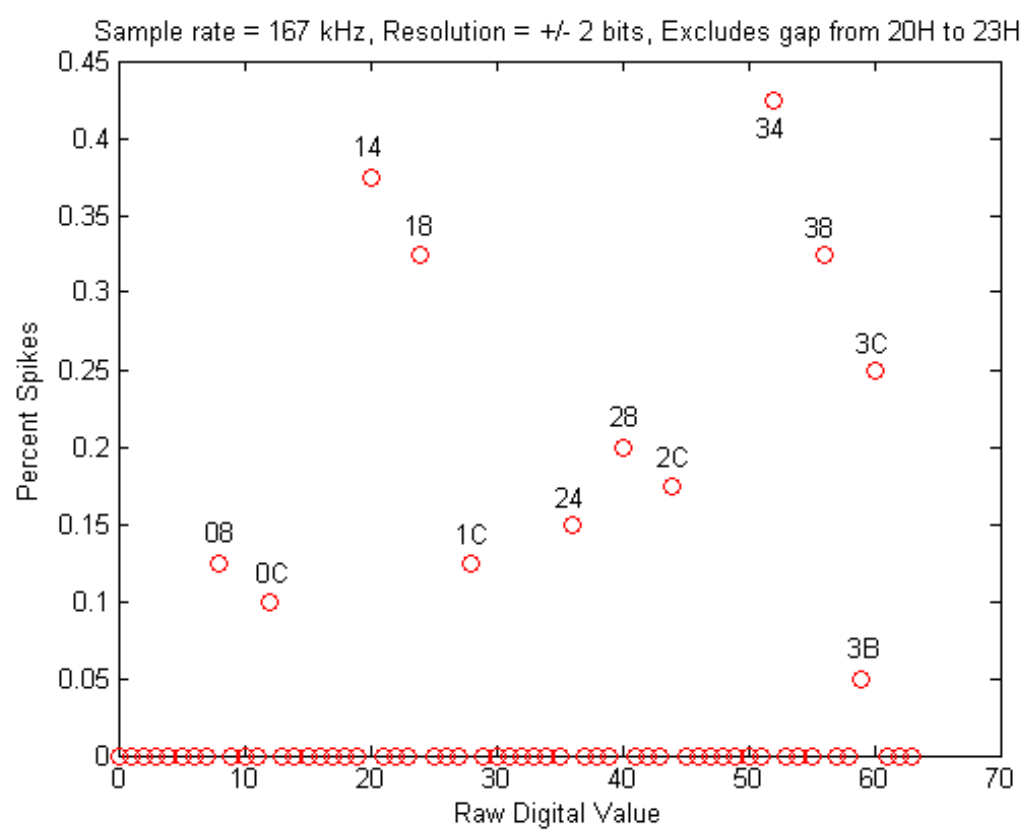

Figure 5-21. Number of digital spikes outside \pm 2-bit window w/ an external $\mathrm{A} / \mathrm{D}$ clock at $167 \mathrm{kHz}$ 


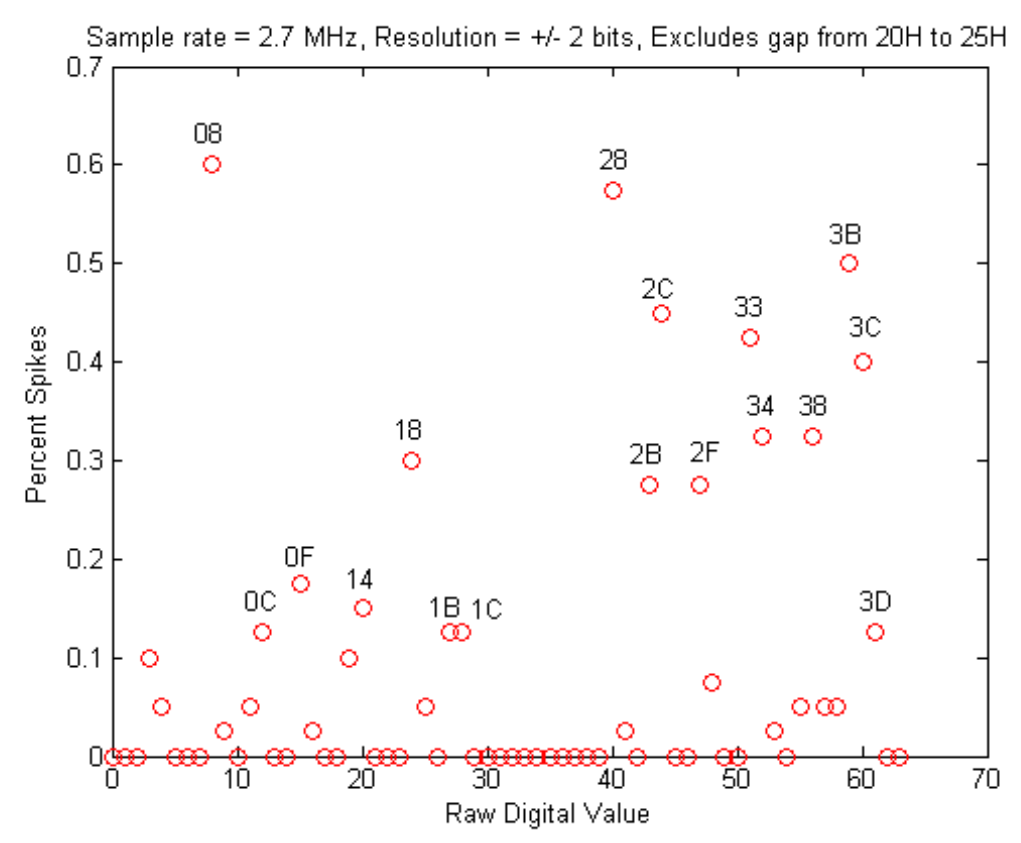

Figure 5-22. Number of digital spikes outside \pm 2-bit window with an internal $\mathrm{A} / \mathrm{D}$ clock at $2.7 \mathrm{MHz}$, $300 \mathrm{~W}$ output power

Note that in order to prevent skewing the plots, the number of spikes during the gap from $20 \mathrm{H}$ to $23 \mathrm{H}$ or $24 \mathrm{H}$ (the upper value depended on sample rate: $23 \mathrm{H}$ for $10 \mathrm{kHz}$ and $167 \mathrm{kHz}$ and $24 \mathrm{H}$ for $2.7 \mathrm{MHz}$ ) was set to zero.

It can be seen from Figure 5-20 and Figure 5-21, and to a lesser extent Figure 5-22, that there are certain digital outputs which tend to spike more often than others. In $\mathrm{HEX}$, these are $\mathrm{X} 4 \mathrm{H}, \mathrm{X} 8 \mathrm{H}$, and $\mathrm{XCH}$ where the $\mathrm{X}$ can be 0,1 , or 2 . There are also certain digital outputs which tend not to spike at all: $\mathrm{X} 3 \mathrm{H}, \mathrm{X} 7 \mathrm{H}$, and $\mathrm{XFH}$. The fact that there are spikes even at $10 \mathrm{kHz}$, and little change from $10 \mathrm{kHz}$ to $167 \mathrm{kHz}$ indicates that the root cause is likely not a timing problem. In fact, observations at a sample rate of $1 \mathrm{kHz}$ showed similar spiking. However, the spiking does get worse as the sample rate is increased from $167 \mathrm{kHz}$ to $2.7 \mathrm{MHz}$.

What is not shown in these plots is the nature of the spikes themselves. Observation of the digital data led to the conclusion that there are specific digital transitions, which cause spikes to specific digital values. This is summarized in Table 5-13.

Table 5-13. Summary of spikes occurring during transitions:

\begin{tabular}{|c|c|c|c|}
\hline \multicolumn{2}{|c|}{ HEX Representation } & \multicolumn{2}{c|}{ Binary Representation } \\
\hline Transition & Spikes & Transition & Spikes \\
\hline $\mathrm{X} 3 \mathrm{H} \leftrightarrow \mathrm{X} 4 \mathrm{H}$ & $\mathrm{X} 0 \mathrm{H}$ and $\mathrm{X} 7 \mathrm{H}$ & $0011 \mathrm{~B} \leftrightarrow 0100 \mathrm{~B}$ & $0000 \mathrm{~B}$ and 0111B \\
\hline $\mathrm{X} 7 \mathrm{H} \leftrightarrow \mathrm{X} 8 \mathrm{H}$ & $\mathrm{X} 0 \mathrm{H}$ and $\mathrm{XFH}$ & $0111 \mathrm{~B} \leftrightarrow 1000 \mathrm{~B}$ & $0000 \mathrm{~B}$ and 1111B \\
\hline $\mathrm{XBH} \leftrightarrow \mathrm{XCH}$ & $\mathrm{X} 8 \mathrm{H}$ and $\mathrm{XFH}$ & $1011 \mathrm{~B} \leftrightarrow 1100 \mathrm{~B}$ & $1000 \mathrm{~B}$ and 1111B \\
\hline $\mathrm{XFH} \leftrightarrow(\mathrm{X}+1) 0 \mathrm{H}$ & $\mathrm{X} 0 \mathrm{H}$ and $(\mathrm{X}+1) \mathrm{FH}$ & $01111 \mathrm{~B} \leftrightarrow 10000 \mathrm{~B}$ & $00000 \mathrm{~B}$ and $01111 \mathrm{~B}$ \\
\hline
\end{tabular}


The conclusion is that spikes tend to occur during rollovers: when consecutive LSBs are all on, and the AD converter tries to turn all these LSBs off and turn on the next MSB (or when one MSB is on and the converter tries to turn that MSB off and turn on all the preceding LSBs). What happens is that the MSB changes but none of the LSBs change (or the LSBs change but the MSB does not) until the next clock cycle. This leads to spikes of specific digital values.

What is also not shown in Figure 5-20- Figure 5-22 is the tendency for each output to oscillate \pm 1 LSB. Not surprisingly, the outputs that tend to spike also tend to oscillate $(\mathrm{X} 4 \mathrm{H}, \mathrm{X} 8 \mathrm{H}$, and $\mathrm{XCH}$ ). This makes sense because these outputs are continuously making the transitions that are prone to spiking. Similarly, the outputs that do not tend to spike are also quite stable (no \pm oscillations). These are the ones that have all consecutive lower bits on, i.e. $\mathrm{X} 3 \mathrm{H}, \mathrm{X} 7 \mathrm{H}$, and $\mathrm{XFH}$. In fact, $1 \mathrm{FH}$ is so stable it remains there when it should not (skipping $20 \mathrm{H}$ to $22+\mathrm{H}$ ). Table 5 summarizes these results.

Table 5-14. Stability of digital outputs

\begin{tabular}{|c|c|c|}
\hline & HEX Representation & Binary Representation \\
\hline Stable Outputs & $\mathrm{X} 3 \mathrm{H}$ & $0011 \mathrm{~B}$ \\
\hline & $\mathrm{X} 7 \mathrm{H}$ & $0111 \mathrm{~B}$ \\
\hline & $\mathrm{XFH}$ & $1111 \mathrm{~B}$ \\
\hline Oscillatory Outputs & $\mathrm{X} 4 \mathrm{H}$ & $0100 \mathrm{~B}$ \\
\hline & $\mathrm{X} 8 \mathrm{H}$ & $1000 \mathrm{~B}$ \\
\hline & $\mathrm{XCH}$ & $1100 \mathrm{~B}$ \\
\hline
\end{tabular}

In summary, it seems apparent that the spikes occur during certain transitions, and that if a particular digital output value is continually making these transitions, it will tend to spike.

\section{Consequences of limitations discovered in A/D converter "flat spot" (accuracy) and "spiking} (stability):

Basically three limitations were revealed in the analog interface test. One is that the ac buffer introduces an offset into the current output. This problem was overcome by injecting current into the ac buffer to compensate for the offset. The second limitation is that the A/D converter contains a "flat spot", as shown Figure 5-16 to Figure 5-19. Fortunately, this has no effect unless operating in this region, which, for lamp current, is above rated power. The final limitation is that some noise is present in the current-mode $\mathrm{A} / \mathrm{D}$ converter, resulting in occasional spikes in the digital output word. These spikes do not significantly affect lamp current regulation since they happen infrequently (on the order of 1 in 1000), however they do adversely effect over-voltage protection. Spikes introduced by the lamp voltage A/D converter caused the control chip to send one-clock-cycle shutdown pulses to the gate driver chip. Therefore, it was necessary to use an external comparator, as shown in Figure 5-23, to provide over-voltage protection. 


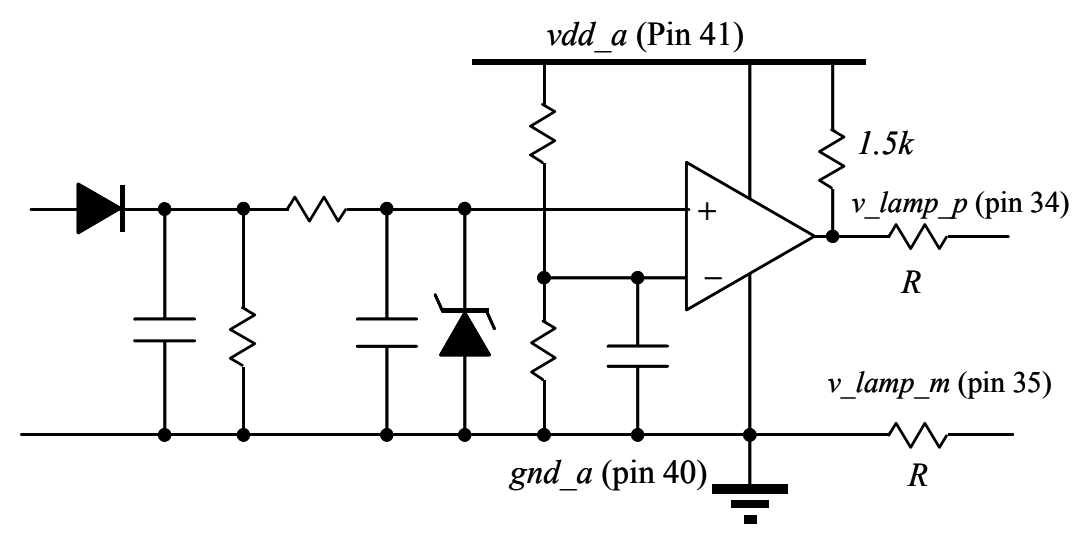

Figure 5-23. External comparator providing over voltage signal to voltage channel to bypass spike caused problem. The output of the comparator keeps low $(\sim 0.2 \mathrm{~V})$ at normal operation; it output $\sim 4.9 \mathrm{~V}$ at over voltage protection mode, which cause lamp voltage channel trigger on-chip over-voltage protection block.

Future versions of the IC will address any A/D converter layout issues which may be causing the non-linearity and noise problems of the converter. In addition, digital averaging or logic will be used to remove outliers from the latched values.

\subsubsection{Digital Control Function}

The objectives of digital control function testing were to verify proper closed loop regulation and start-up behavior. Good closed loop regulation requires proper operation of the phase controller, peak detector, and compensator. A successful start-up requires proper operation of the start-up controller, DCO controller, and mode controller, as well as the phase controller. Over voltage shutdown was also tested. Steady-state waveforms of closed-loop operation at varying power levels as well as DCO operation are presented, along with a table summarizing the results. Waveforms of several start-ups are also presented.

\section{Closed-loop regulation:}

The HID ballast shown in Figure 3-41 was used as a test bed by removing all the FPGA and analog interfaced related circuit. Table 5-15 summarizes results from closed-loop operation at $1.28,2.56,3.84$, and 4.48A current commands as well as DCO operation with resistive load. Bus voltage, $\mathrm{V}_{\mathrm{b}}$, was $300 \mathrm{~V}$, ADC sample rate was $16 \mathrm{x}$ switching frequency. In this table, Current Cmd is the user-specified digital value, Pin is the output power of the ballast power source, $\mathrm{T}$ is the period of the lamp current, Ilamp (scope) is the actual measured lamp current, Ilamp (digital) is the digital lamp current from the A/D converter, Iind (scope) is the measured inductor current, Phase (scope) is the measured phase angle between midpoint voltage and inductor current, Phase Cmd (digital) is the digital phase command, and Comparator Output Duty Cycle is the positive duty cycle. 
Table 5-15. Summary of closed-loop test results at $300 \mathrm{~V}$ dc bus voltage and $16 x f_{s}$ ADC clock:

\begin{tabular}{|c|c|c|c|c|c|c|c|c|}
\hline $\begin{array}{c}\text { Current } \\
\text { Cmd }\end{array}$ & $\begin{array}{c}\text { Pin } \\
{[\mathrm{W}]}\end{array}$ & $\begin{array}{c}\mathrm{T} \\
{[\mu \mathrm{s}]}\end{array}$ & $\begin{array}{c}\mathrm{I}_{\text {lamp }} \\
(\mathrm{scope}) \\
{[\mathrm{A}]}\end{array}$ & $\begin{array}{c}\text { Ilamp } \\
(\text { digital }) \\
{[\mathrm{A}]}\end{array}$ & $\begin{array}{c}\text { Iind } \\
{[\mathrm{A}]}\end{array}$ & $\begin{array}{c}\text { Phase } \\
(\text { scope }) \\
{[\mathrm{deg} .]}\end{array}$ & $\begin{array}{c}\text { Phase Cmd } \\
(\text { digital }) \\
{[\mathrm{deg} .]}\end{array}$ & $\begin{array}{c}\text { Comparator } \\
\text { Output } \\
\text { Duty Cycle } \\
{[\%]}\end{array}$ \\
\hline $\mathrm{DCO}$ & 26 & 6.0 & 1.08 & $06 \mathrm{H}=0.96$ & 2.62 & 81 & $\mathrm{FFH}=90$ & 51.9 \\
\hline $1.28 \mathrm{~A}$ & 56 & 6.6 & 1.64 & $09 \mathrm{H}=1.44$ & 3.90 & 76 & $\mathrm{~F} 6 \mathrm{H}=87$ & 51.2 \\
\hline $2.56 \mathrm{~A}$ & 190 & 7.1 & 2.90 & $10 \mathrm{H}=2.56$ & 7.00 & 71 & $\mathrm{DEH}=78$ & 50.6 \\
\hline $3.84 \mathrm{~A}$ & 440 & 7.4 & 4.30 & $17 \mathrm{H}=3.84$ & 10.3 & 60 & $\mathrm{C} 3 \mathrm{H}=68$ & 50.3 \\
\hline $4.48 \mathrm{~A}$ & 570 & 7.45 & 4.90 & $1 \mathrm{CH}=4.48$ & 11.7 & 58 & $\mathrm{BAH}=65$ & 50.3 \\
\hline
\end{tabular}

Table 5-15 shows that the error between phase command and actual phase is between 7 and $11^{\circ}$, with the actual phase being less than the commanded phase. The accuracy of the lamp current A/D conversion has previously been addressed and is not discussed in detail here, particularly since it is probable that the scope measurements and the digital recordings were not taken at exactly the same instant. It is noted however that the error as a percentage of the actual lamp current is $9 \%-12 \%$, with the actual current being greater than the digital value. Figure 5-25 shows four-waveform plots of steady-state operation at each of the operating points listed in Table 5-15. Figure 5-26 shows steady-state operation of the HID lamp at near rated power. The phase error between commanded phase and actual phase is approximately $3^{\circ}$, around $5 \%$ of phase command.

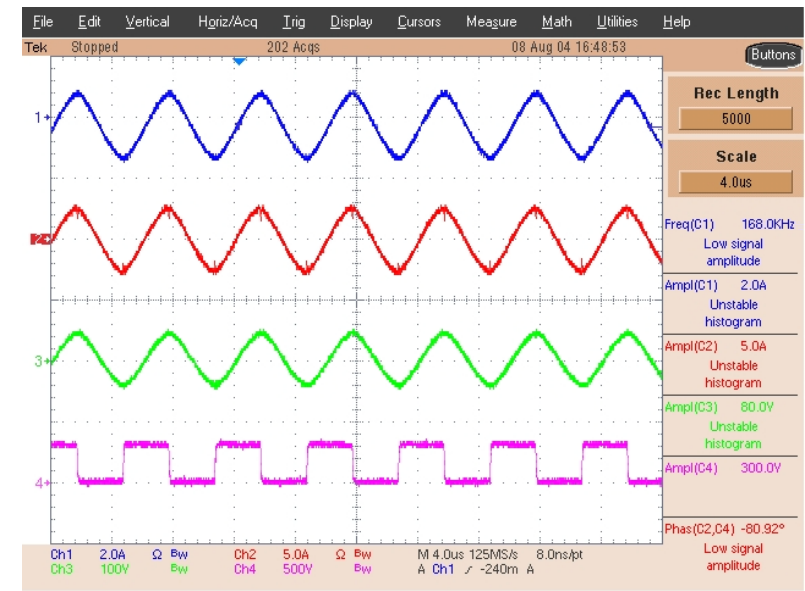

Figure 5-24. Steady state waveforms at DCO mode, with 300V dc bus voltage and 16x AD sample rate. Ch1: lamp current; $C h$ 2: inductor current; $C h$ 3: lamp voltage; Ch 4: midpoint voltage 


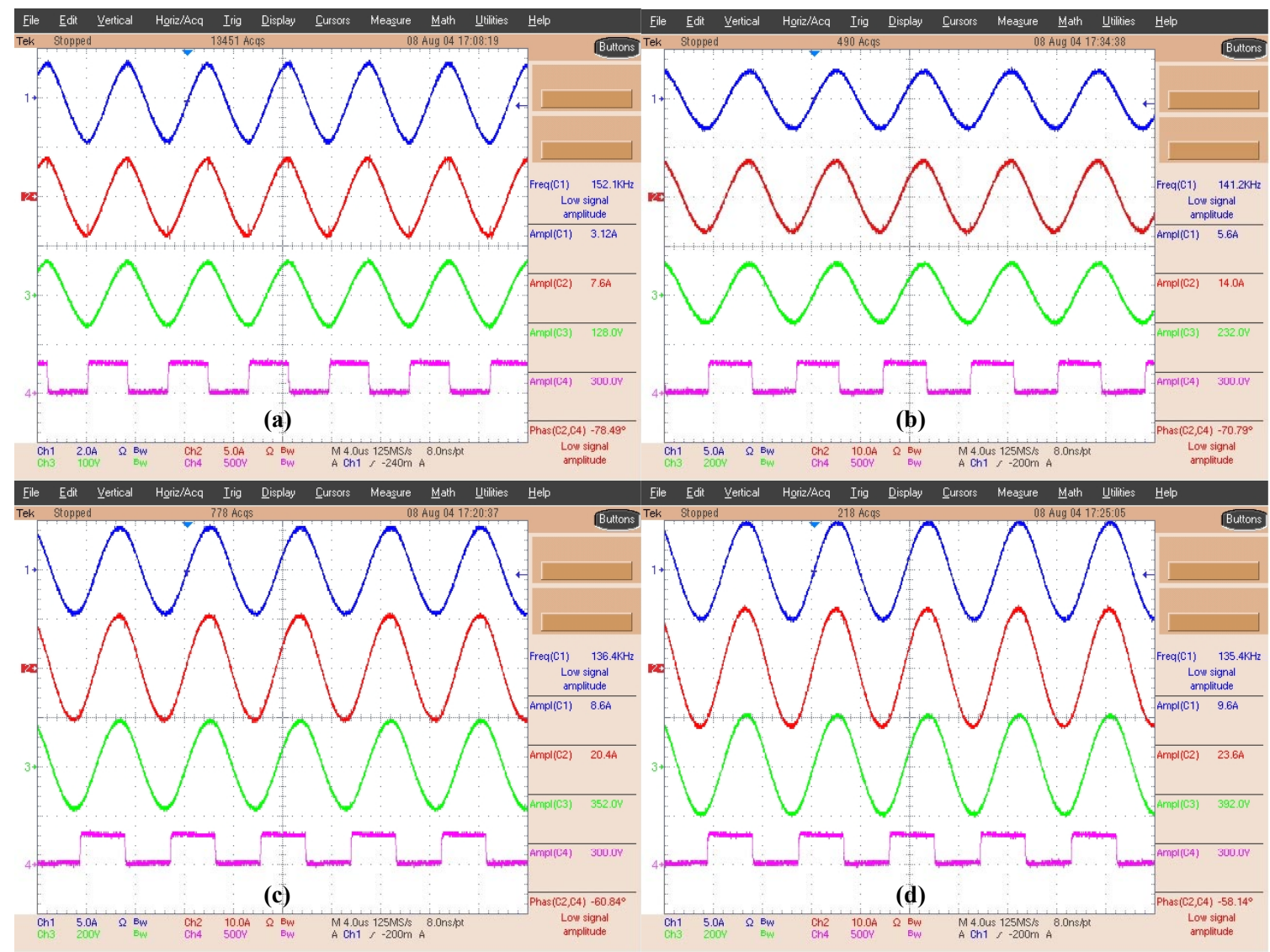

Figure 5-25. Steady state waveforms at different current command: a) $1.28 \mathrm{~A}$, b) $2.56 \mathrm{~A}$, c) $3.84 \mathrm{~A}$, and d) 4.48A; Ch1: lamp current; Ch 2: inductor current; Ch 3: lamp voltage; Ch 4: midpoint voltage

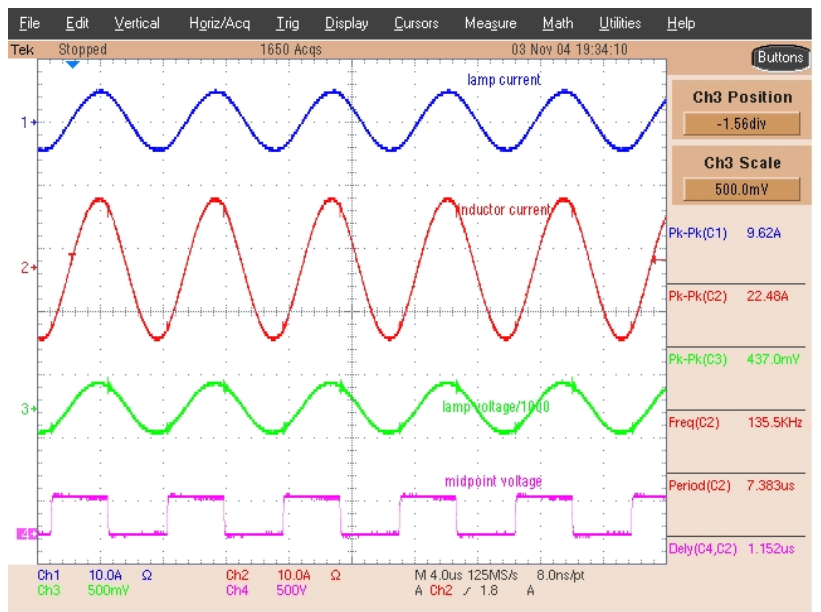

Figure 5-26. Steady state operation of 400W HID lamp with 300V DC bus voltage, 4.8A lamp peak current, $136 \mathrm{kHz}$ operation frequency, $58^{\circ}$ measured phase and $61^{\circ}$ phase command 


\section{Over voltage protection:}

By using external comparator, the spiking problem was bypassed and the on-chip over-voltage protection function block can be tested. Figure 5-27 demonstrates over-voltage protection operation with the over-voltage limit set near 3,500 V. As shown, the ballast controller repeats ignition sequence indefinitely until the lamp can ignite below the over-voltage limit, which provides a safe and reliable operation of the ballast.

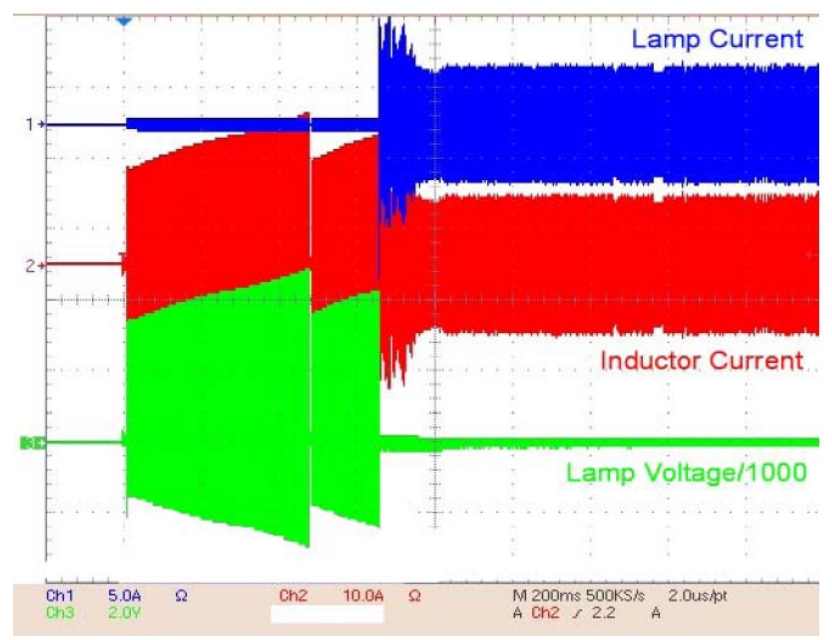

Figure 5-27. Over voltage protection showing a repeated ignition attempts of HID lamp until it ignited below preset $3.5 \mathrm{kV}$ protection level

\section{Start-up behavior:}

During start-up, the mode controller operates the chip in DCO mode for $10 \mathrm{~ms}$, then switches to phase control mode with the phase command provided by the start-up controller. The start-up controller sweeps the phase command from the start phase specified by the st phase_sel switch $\left(90^{\circ}\right.$ in this case) down to the minimum phase specified by the min $\_$phase_sel switch $\left(17^{\circ}\right.$ in this case) in steps of one LSB (about $0.35^{\circ}$ ). The duration of operation at each decremental phase setting is set by the swp_sel switch and is here is $5 \mathrm{~ms}$. If at any time during the sweep the peak detector detects lamp peak current greater than the threshold value set by the ith_sel switch (equal to the current command in this case), then the sweep is stopped and the controller enters closed loop regulation where the phase command is provided by the compensator.

Figure 5-28 shows start-up sequence with a resistive load and a 2.56A current command. The phase sweep is obvious, but the $10 \mathrm{~ms}$ DCO period is barely discernible since there is no step in current after the $10 \mathrm{~ms}$ DCO period due to the constant load and smaller current reference. The step becomes more evident if HID lamp is used, as shown in Figure 5-29. Once the lamp ignites, it goes through a very short glow-to-arc phase, followed by a warm-up period. During warm-up, the regulator maintains near constant current despite changes to the lamp impedance. Figure 5-30 
shows a detail view of the challenging lamp dynamics during the glow-to-arc phase. The lamp current initially contains higher order harmonics and oscillates between rectifying behavior prior to stable arc operation. The phase controller is unaffected by these dynamics as it tracks constant phase despite variations in the tank input impedance. Changes in the frequency of the midpoint voltage are observed as the phase controller maintains an approximately constant phase (due to the small compensator gain used, the phase command is held approximately constant over the several cycles shown). It should be noted that during the glow-to-arc operating conditions, the lamp current peaks are not a good representation of the lamp RMS current. A simple improvement would be to perform a cycle-by-cycle average of the rectified current waveform as a closer approximation to the RMS value with relatively little processing overhead. This would provide higher currents during initial operation and could help to force the lamp out of the glowto-arc mode more quickly.

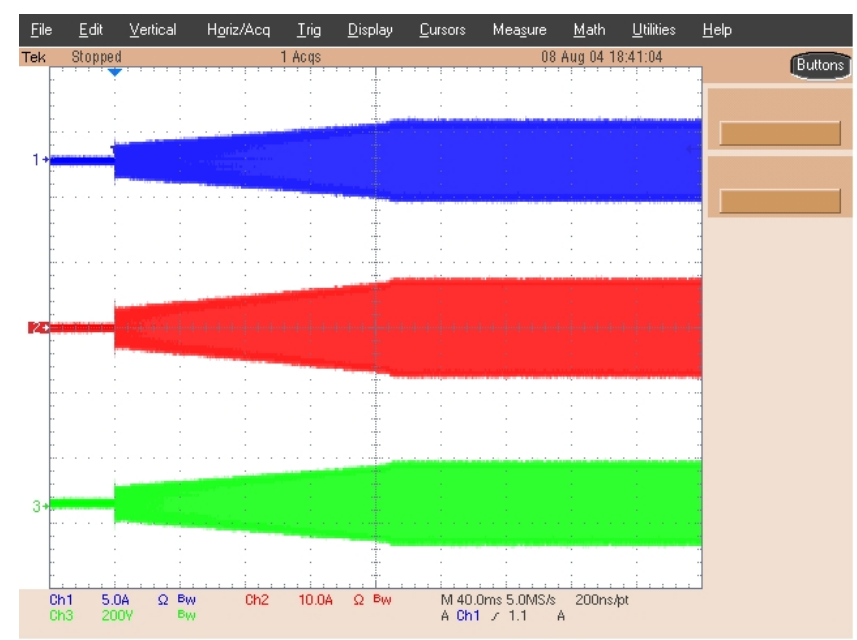

Figure 5-28. Resistor load start-up waveforms with 2.56A current command, ch1: output current, ch2: inductor current, ch3: load voltage, ch4: midpoint voltage 


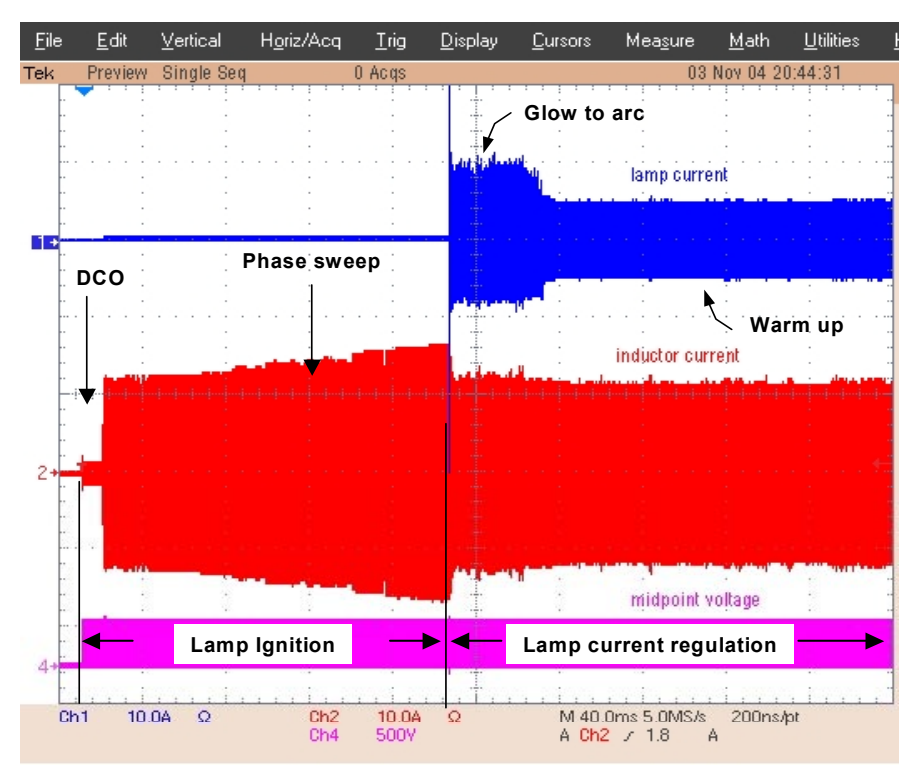

Figure 5-29. HID lamp ignition sequence with operation modes labeled. De bus voltage $=300 \mathrm{~V}$, current commend $=\mathbf{4 . 8 A}$

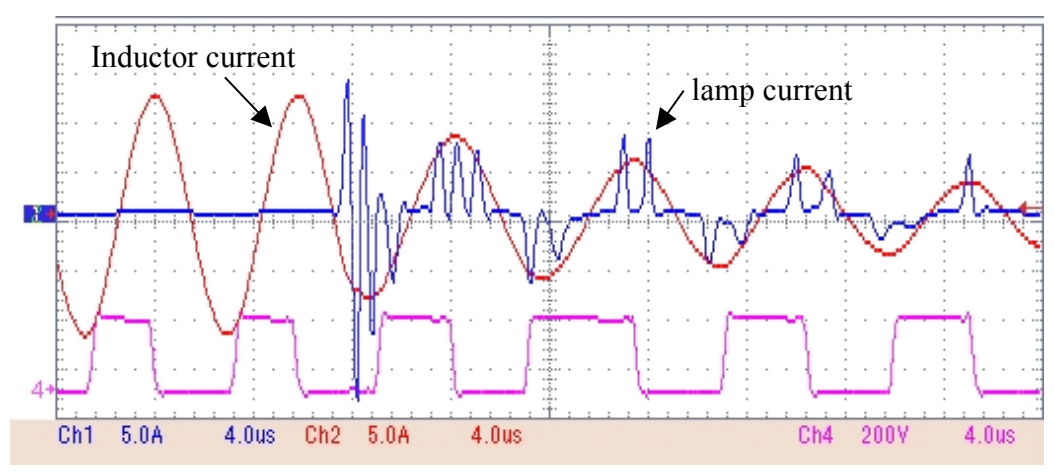

Figure 5-30. HID lamp ignition dynamics in the first few cycle

\subsection{Ballast Controller and Gate Drive IC Performance in Demo Ballasts}

The objective of the test is to demonstrate the feasibility of developed chip set for the high frequency ballast platform. HID and LFL ballasts were selected as demonstration ballasts. The experimental setup of 400W HID ballast prototype is shown in Figure 3-51 with a schematic in Figure 3-41. The experimental setup of 32W LFL ballast prototype is shown in Figure 3-52. The performance of HID ballast has been discussed in section 5.2.3; the following section will be focused on the performance test of LFL ballast.

The power stage is a typical half-bridge ballast with a $320 \mathrm{~V}$ bus voltage and $32 \mathrm{~W}$ LFL load. All the control, gate drive and protection functions were fulfilled by the ballast controller and gate drive ICs. Since the chip set was tested thoroughly in sections 5.1 and 5.2, the system integration 
becomes very easy. To verify proper ballast operation at near rated power an $800 \Omega$ resistor was used first. The test results are shown in Figure 5-31. Initially ballast operates at DCO mode with $72 \mathrm{kHz}$ switching frequency for $\sim 8 \mathrm{~ms}$. Following the DCO mode is the phase sweep mode. Due to a $90^{\circ}$ starting phase used, a frequency jump was noticed. The smooth transition from DCO mode to phase sweep mode can be addressed in future version. The transition from phase sweep to close loop control was very smooth, as discussed in section 3.2.4. Figure 5-31(b) shows the steady state operation. The measured current was $151.6 \mathrm{~mA}_{\mathrm{rms}}$, around $1 \%$ greater than $150 \mathrm{~mA}_{\mathrm{rms}}$ reference.

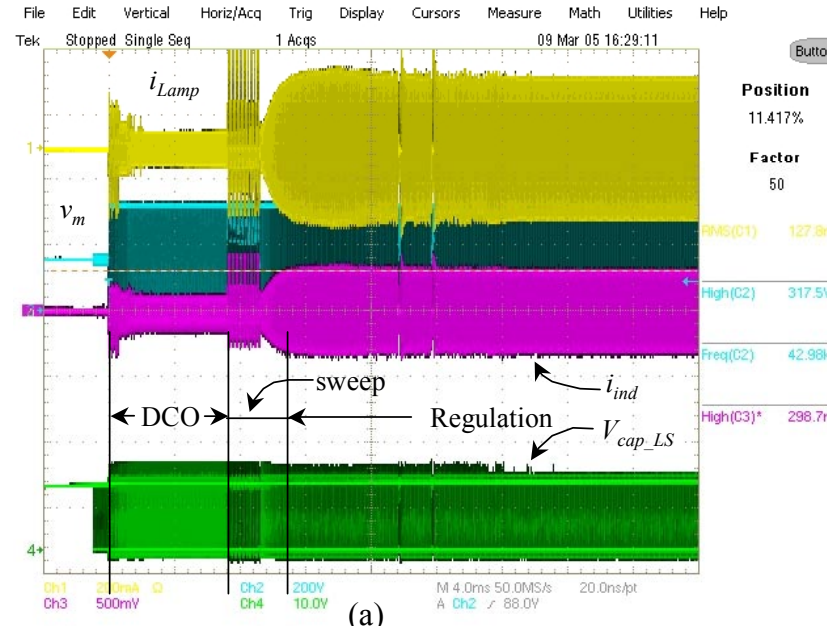

(a)

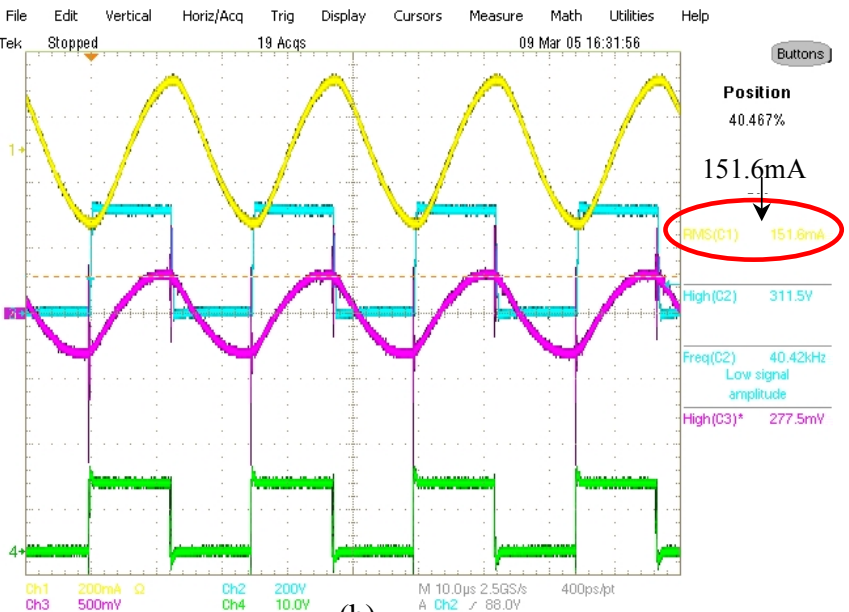

(b)

Figure 5-31. Demo ballast performance for resistor load at near rated power: (a) start up sequence with expected operation modes; (b) steady state operation with $1 \%$ regulation error; ch1: lamp current, 200mA/div; ch2: midpoint voltage, 200V/div; ch3: sensed inductor current from a CT loaded by $20 \Omega, 0.5 \mathrm{~V} / \mathrm{div} ; \mathrm{ch} 4$ : $\mathrm{LS}$ cap voltage, $10 \mathrm{~V} / \mathrm{div}$

After resistive load test the demo ballast operated with an energy efficient LFL lamp F32T8, as shown in Figure 5-32. The captured lamp starting and steady state waveforms are shown in Figure 5-33(a) and (b), respectively. The lamp ignition was very smooth with expected operation modes. The steady state regulation was again very good, around $5 \%$ greater than the current reference. The experimental results also demonstrated a flawless operation of gate drive IC in a dynamic environment with a negative highly non-linear load and significant radiated EMI. As shown in Figure 5-34, two ICs finished communication and masked before the highly noised falling and rising transitions based on the state machines in Figure 3-14. 


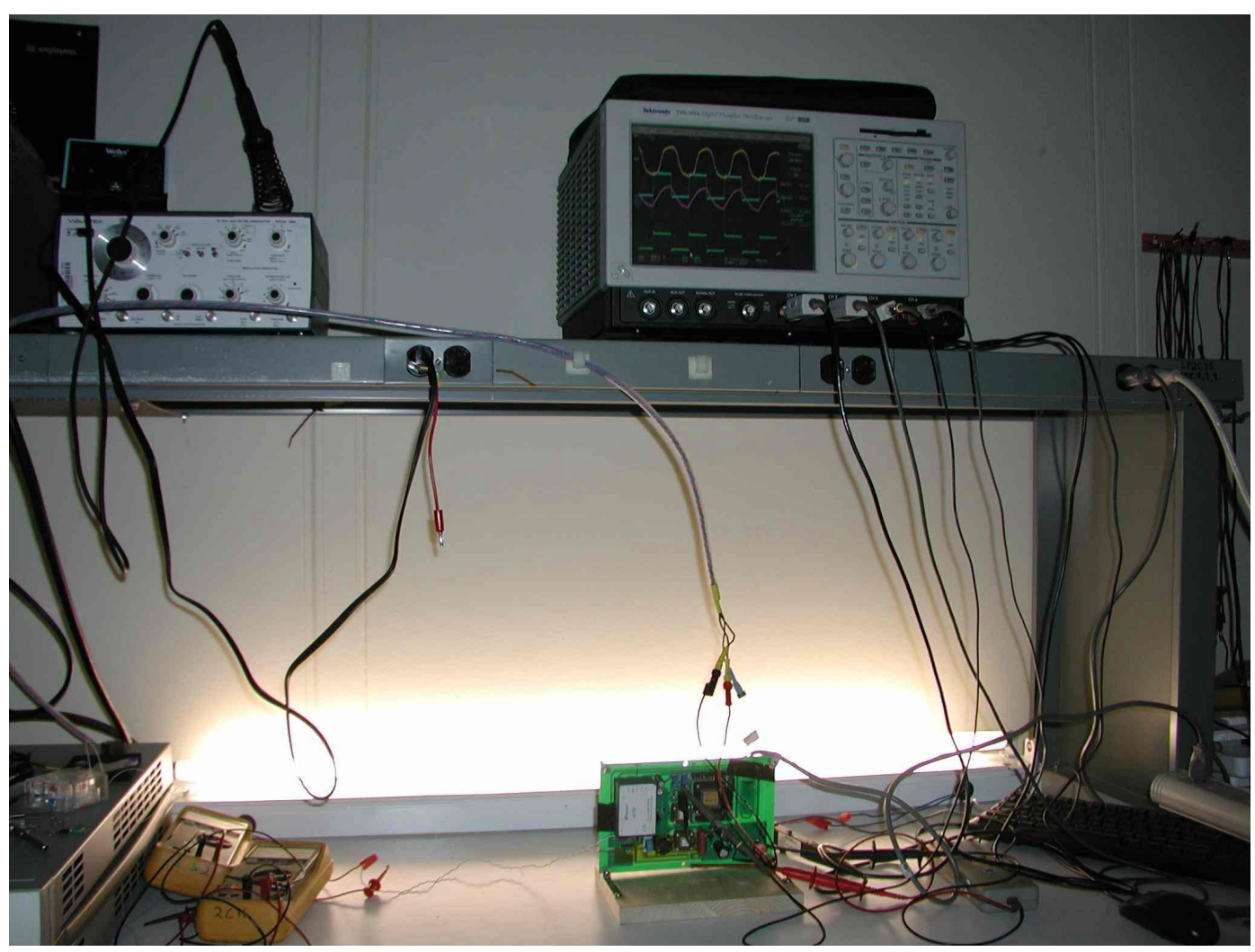

Figure 5-32. Photograph demonstrating steady-state operation of demo ballast driving a $32 \mathrm{~W}$ T8 energy efficient LFL lamp
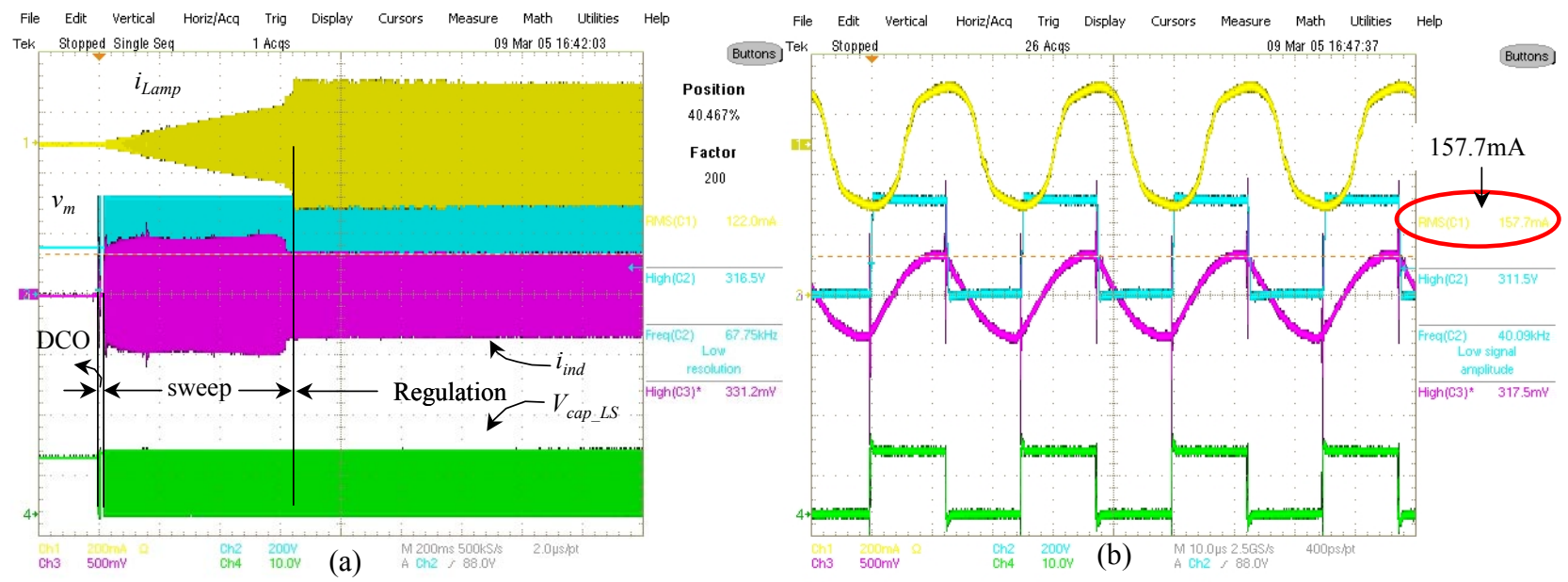

Figure 5-33. Experimental results showing performance of demo ballast driving a $32 \mathrm{~W}$ T8 LFL lamp at near rated power: (a) start up sequence with expected operation modes; (b) steady state operation with $5 \%$ regulation error; ch1: lamp current, $200 \mathrm{~mA} / \mathrm{div}$; $\operatorname{ch} 2$ : midpoint voltage, $200 \mathrm{~V} / \mathrm{div}$; ch3: sensed inductor current from a CT loaded by $20 \Omega$, $0.5 \mathrm{~V} / \mathrm{div}$; ch4: $\mathrm{LS}$ cap voltage, $10 \mathrm{~V} / \mathrm{div}$ 


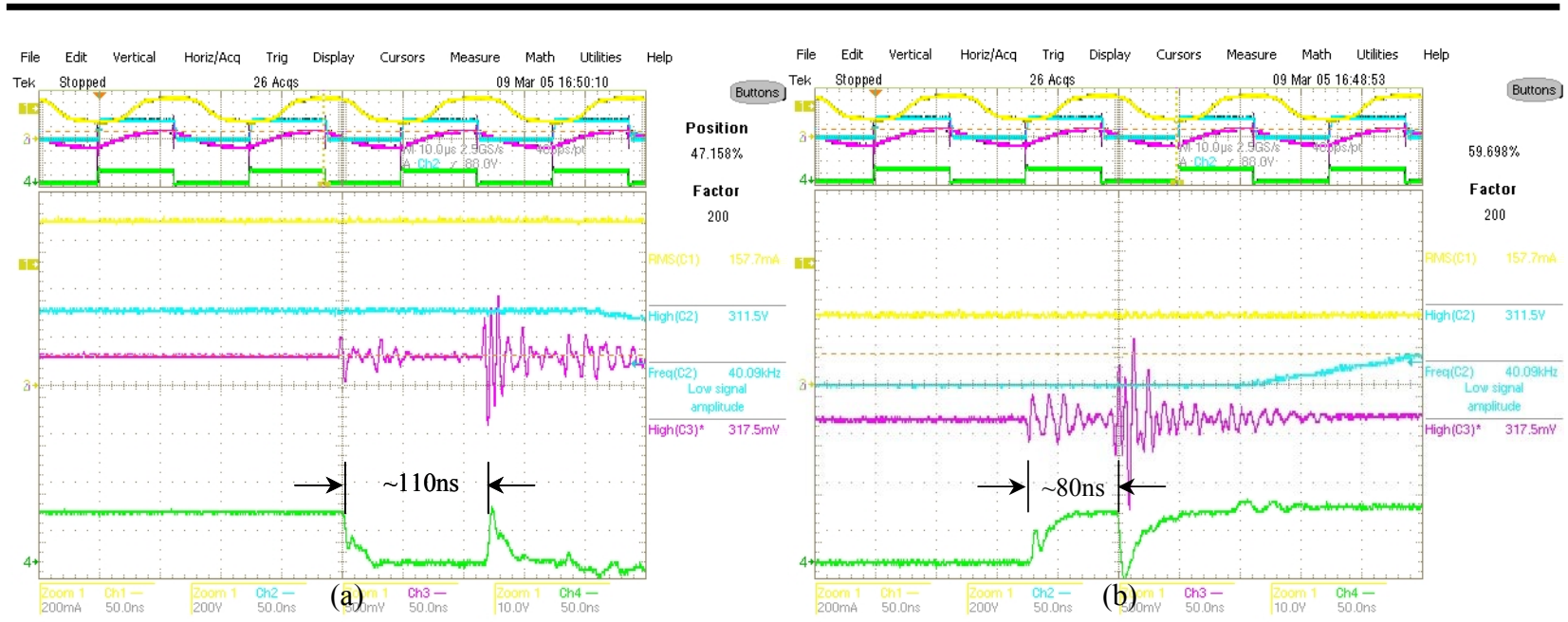

Figure 5-34. Experimental results demonstrating robust operation of the dual LVIC gate drive prototype IC: a) falling transition; b) rising transition; ch1: lamp current, $200 \mathrm{~mA} / \mathrm{div}$; ch2: midpoint voltage, 200V/div; ch3: sensed inductor current from a CT loaded by $20 \Omega, 0.5 \mathrm{~V} / \mathrm{div} ;$ ch4: LS cap voltage, $10 \mathrm{~V} / \mathrm{div}$ 


\section{CONCLUSION}

A new approach for driving energy efficient lighting products has been successfully developed and demonstrated through dual LVIC gate drive IC, ballast control IC and demo ballast platform.

The prototype gate drive IC was fabricated in a low-cost $15 \mathrm{~V}, 0.8 \mu \mathrm{m}$ CMOS process and includes the entire core blocks necessary for independent operation. The design offers the benefits of minimal external components, built-in power supply, low-cost IC fabrication and simple extensions for adaptive dead time control and high frequency operation. It has the potential of delivering a low-cost alternative to HVIC gate drives. Experimental results demonstrate robust performance driving a 320V half-bridge ballast with T8, 32W LFL lamp.

The prototype ballast controller IC was fabricated in a low-cost standard CMOS process and includes fully integrated functions of programmable ignition and transition to normal operation, ac lamp current regulation, over-voltage protection and dimming control. Phase control provides self-tuning to the ballast resonant frequency, inherent ZVS operation, and simplified dynamics for current regulation. The primarily digital approach provides programmability for broader application, insensitivity to process and temperature variations, few external components, and ease of re-use and adaptation to other platforms and IC technologies. Experimental results are provided demonstrating IC operation in 400W HID and 32W LFL ballast applications. One cycle ZVS operation together with fast current loop control and high frequency would benefit low wattage HID lamps for acoustic resonance-free operation.

The developed approach would benefit a family of lighting products through the potential advantages of reduced system cost, reduced ballast size, universal application to a wide range of lamp technologies, and network-friendly interface for networked lighting system.

Further work could include: 1) addressing issues discovered in the IC tests; 2) more practical design with test pin removal (Figure 6-1); 3) an improved IC design with smaller IC size, lower power consumption, higher current handling capability (gate/cap drive switches), improved noise immunity and better performance; 4) applications in the networked lighting systems.

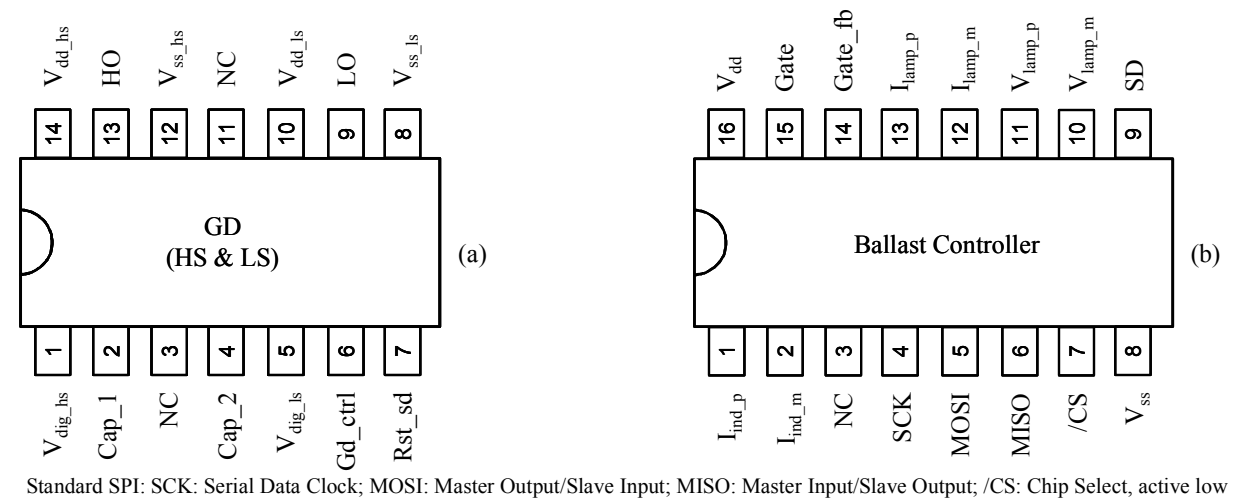

Figure 6-1. Potential pin assignment of a) LVIC gate drive and b) Ballast controller IC 


\section{REFERENCES}

[1]. H.W. Lord, "Pulse Transformers," IEEE Trans. on Magnetics, Vol. MAG-7, No.1, March 1971, pp. 17-28.

[2]. F. A. Schoofs, C. N. Dupont, “A 700-V interface IC for power bridge circuits," IEEE Journal of Solid State Circuits, Vol. 25, No. 3, pp. 677-683, Jun 1990.

[3]. Application note: HV Floating MOS-Gate Driver ICs. International Rectifier, AN978, June 2003. Available at www.irf.com.

[4]. P. Shihong, T. M. Jahns, "A self-boost charge pump topology for a gate drive high-side power supply," in Proc. IEEE Applied Power Electronics Conference and Exposition, Feb. 2003, pp. 126-131.

[5]. J. P. Karst, K. Hoffmann, "Transductor based high speed gate drive," in Proc. IEEE Power Electronics Specialists Conference, June 2004, pp. 100-104

[6]. L. R. Nerone, "A Novel MOSFET Gate Driver for the Complementary Class D Converter," in Proc. IEEE Applied Power Electronics Conference and Exposition, March 1999, pp. $760-763$.

[7]. Y. Yin, R. Zane, "Dual Low Voltage IC Based High and Low Side Gate Drive," Proceedings IEEE Applied Power Electronics Conference and Exposition, February 2004, pp 1033-1038.

[8]. H. Träff, "A novel approach to high speed CMOS current comparators," Electron. Lett., Vol. 28, No. 3, pp. 310-312, Jan. 1991.

[9]. W-H Ki, J. Shi, E. Yau, P. K. T. Mok, J. K. O Sin, "Phase-Controlled Dimmable Electronic Ballast for Fluorescent Lamps," in Proc. Power Electronics Specialists Conference, June, 1999, pp. 1121-1125.

[10]. Virtex-II V2MB1000 Development Board User's Guide, Memec, Version 1.3, 2002,

[11]. W. W. S. Chan, P. K. T. Mok, A. T. K. Ng, W-H. Ki, and J. K. O. Sin, "IC Controller for Phase-Controlled Dimmable Compact Fluorescent Lamps with Closed-Loop Control," in Proc. ISCAS 2000, pp. 503-506.

[12]. T.J. Ribarich, J.J. Ribarich, “A new control IC for dimmable high frequency electronic ballast," in Proc. IEEE Applied Power Electronics Conference and Exposition, Vol. 2, pp. 713 -719, March 1999.

[13]. Y. Yin, R. Zane, "Digital Phase Control for Resonant Inverters,” IEEE Power Electronics Letters, Vol. 2, No. 2, pp. 51-53, June 2004.

[14]. Y. Yin, R. Zane, "Digital Controller Design for Electronic Ballasts with Phase Control," in Proc. IEEE Power Electronics Specialists Conference, Jun. 2004, pp. 1855-1860.

[15]. Y, Yin, "Modeling and IC-based control of high frequency electronic ballasts," Ph.D. dissertation, University of Colorado, 2004.

[16]. Y. Yin, R. Zane, "Integrated Results for Dual Low Voltage IC Based High and Low Side Gate Drive," in Proc. IEEE Applied Power Electronics Conference and Exposition, Mar. 2005.

[17]. Y. Yin, M. Shirazi, R. Zane, "Fully Integrated Ballast Controller with Digital Phase Control," in Proc. IEEE Applied Power Electronics Conference and Exposition, Mar. 2005. 


\section{ACKNOWLEDGEMENTS}

This project and report is a joint effort of the following staff of the GE Global Research Center, GE Consumer \& Industrial, and the Colorado Power Electronics Center at University of Colorado. The submitter would like to acknowledge all the contribution from the core team at GE GRC and CoPEC for their work on this project: Dr. Richard Zhang, the Principle Investigator, Dr. Ljubisa Stevanovic, Dr. Fengfeng Tao, Rebecca Voelker, Frank Mueller, Dr. Regan Zane and Dr. Yan Yin. We would also like to gratefully acknowledge the contributions of GE Consumer \& Industrial's Dr. Louis Nerone and Dr. Didier Rouaud for their help with the IC and ballast prototyping. The authors would also like to thank sincerely the Department of Energy, without whose support most of this present work would not have been possible. 


\section{LIST OF ACRONYMS AND ABBREVIATIONS}

A
ASIC
C
CMOS
CoPEC
DC
DCO
EMI
ESD
F
FET
FSM
GE
GECP
GRC
H
HSIC
Hz
IC
IEEE
LLD
MOSIS

$\mathrm{n}$

OTA

PWM

$\mathrm{R}$

SNR

$\mu$

UVLO

$\mathrm{W}$

ZVS

\author{
Amps \\ Application Specific Integrated Circuit \\ Coulomb \\ Complementary Metal-Oxide Semiconductor \\ Colorado Power Electronics Center \\ Direct Current \\ Digitally-Controlled-Oscillator \\ Electromagnetic Interference \\ Electrostatic Discharge \\ Farad \\ Field Effect Transistor \\ Finite-state-machine \\ General Electric \\ General Electric Consumer Products \\ Global Research Center \\ Henry \\ High-side IC \\ Hertz \\ Integrated Circuit \\ Institute of Electrical and Electronics Engineers \\ Light Lumen Depreciation \\ Metal Oxide Semiconductor Implementation Service. MOSIS is a multiproject \\ fabrication service run by ARPA (The Advanced Research Projects Agency). \\ Nano- $\left(10^{-9}\right)$ \\ Operational transconductance amplifier \\ Pulse-Width Modulation \\ Resistance \\ Signal-Noise Ratio \\ Micro- $\left(10^{-6}\right)$ \\ Under-Voltage Lock-Out \\ Watts \\ Zero-Voltage-Switching
}

